\title{
SECOND ORDER PERIODIC DIFFERENTIAL OPERATORS. THRESHOLD PROPERTIES AND HOMOGENIZATION
}

\author{
M. SH. BIRMAN AND T. A. SUSLINA
}

\begin{abstract}
The vector periodic differential operators (DO's) $\mathcal{A}$ admitting a factorization $\mathcal{A}=\mathcal{X}^{*} \mathcal{X}$, where $\mathcal{X}$ is a first order homogeneous $\mathrm{DO}$, are considered in $L_{2}\left(\mathbb{R}^{d}\right)$. Many operators of mathematical physics have this form. The effects that depend only on a rough behavior of the spectral expansion of $\mathcal{A}$ in a small neighborhood of zero are called threshold effects at the point $\lambda=0$. An example of a threshold effect is the behavior of a DO in the small period limit (the homogenization effect). Another example is related to the negative discrete spectrum of the operator $\mathcal{A}-\alpha V$, $\alpha>0$, where $V(\mathbf{x}) \geq 0$ and $V(\mathbf{x}) \rightarrow 0$ as $|\mathbf{x}| \rightarrow \infty$. "Effective characteristics", such as the homogenized medium, effective mass, effective Hamiltonian, etc., arise in these problems. The general approach to these problems proposed in this paper is based on the spectral perturbation theory for operator-valued functions admitting analytic factorization. Most of the arguments are carried out in abstract terms. As to applications, the main attention is paid to homogenization of DO's.
\end{abstract}

\section{Contents}

$\S 0$. Introduction

Chapter 1. Operator families admitting factorization

640

$\S 1$. Quadratic pencils of the form $X(t)^{*} X(t)$

$\S 2$. Auxiliary material

$\S 3$. Estimates for the difference of resolvents on the contour

$\S 4$. Threshold approximations

$\S 5$. Approximation for the operator-valued function $\left(A(t)+\varepsilon^{2} I\right)^{-1}$

Chapter 2. Periodic differential operators in $L_{2}\left(\mathbb{R}^{d} ; \mathbb{C}^{n}\right)$

$\S 1$. Main definitions. Preliminaries

$\S 2$. Direct integral expansion for the operator $\mathcal{A}$

$\S 3$. Incorporation of the operators $\mathcal{A}(\mathbf{k})$ into the pattern of $\S 1$, Chapter $1 \quad 662$

Chapter 3. Effective characteristics near the lower edge

of the spectrum

$\S 1$. Effective matrices and the effective DO

$\S 2$. Behavior of the resolvent $\left(\mathcal{A}+\varepsilon^{2} I\right)^{-1}$ as $\varepsilon \rightarrow 0$

$\S 3$. Behavior of the generalized resolvent as $\varepsilon \rightarrow 0$

Chapter 4. Homogenization problems for periodic DO's $\quad 673$

$\S 1$. Statement of the problem

$\S 2$. Approximation of the resolvent in the operator norm $\quad 675$

$\S 3$. Weak convergence of solutions and flows

$\S 4$. Convergence of solutions and flows (part 2)

2000 Mathematics Subject Classification. Primary 35P99, 35Q99.

Key words and phrases. Periodic operators, threshold effect, homogenization.

Supported by RFBR (grant no. 02-01-00798). 
Comments on Chapter 4

Chapter 5. Applications of the general method. The case where $f=\mathbf{1}_{n}$

$\S 1$. The periodic acoustic and Schrödinger operators

$\S 2$. The operator of elasticity theory on $\mathbb{R}^{d}, d \geq 2$

Comments on Chapter 5

Chapter 6. Applications of the general method. The case where $f \neq \mathbf{1}_{n}$

$\S 1$. Preliminary remarks

$\S 2$. The operator $\mathcal{L}(\epsilon, \nu)=\operatorname{rot} \epsilon^{-1} \operatorname{rot}-\nabla \nu \operatorname{div}$

$\S 3$. Homogenization for a periodic Maxwell system with $\mu=1$

\section{$\S 0$. INTRODUCTION}

0.1. The periodic differential operators (DO's) on $\mathbb{R}^{d}, d \geq 1$, can be partially diagonalized with the help of the Gelfand transformation (see Chapter 2). Under this approach, the initial DO is represented as the direct integral of a family of DO's; each operator in this family acts on the cell of periods $\Omega$ glued into a torus. This family depends on a parameter $\mathbf{k} \in \mathbb{R}^{d}$ (called the quasimomentum). We consider the semibounded selfadjoint DO's.

Further analysis (the Floquet-Bloch decomposition) is possible for operators acting in $L_{2}(\Omega)$ and having compact resolvent that depends on $\mathbf{k}$ continuously. This condition is fulfilled for many DO's of mathematical physics. In this case, the spectrum of the initial DO has a band structure. It is convenient to assume that the lower edge of the spectrum coincides with the point $\lambda=0$. In some situations, it suffices to know the approximate spectral expansion of the initial DO near the lower edge of the spectrum. In such cases we talk about the threshold effects at the point $\lambda=0$. Threshold effects can be related to the edges of internal spectral gaps, but we shall only deal with the threshold $\lambda=0$. Usually, it is not an easy task to detect whether a given effect is threshold. For instance, we mention the question about the discrete spectrum that arises to the left of the point $\lambda=0$ when a periodic DO is perturbed by a negative potential vanishing at infinity. If the potential does not decay too rapidly, then the threshold effect dominates; on the contrary, if a perturbation decays rapidly, then the main role is played by the high-energy part of the initial DO that corresponds to large values of $\lambda$. Another important example of the threshold effect at the point $\lambda=0$ is the behavior of a periodic DO in $L_{2}\left(\mathbb{R}^{d}\right)$ in the small period limit. In what follows, we pay the main attention to this problem.

0.2. One of our goals is to give a description, concise and convenient for applications, of the spectral characteristics of periodic DO's near the threshold $\lambda=0$. By using partial diagonalization, we reduce the problem to some questions of perturbation theory for the discrete spectrum. The difficulties are related to the fact that, usually, the unperturbed eigenvalue is multiple and the parameter $\mathbf{k}$ is multidimensional. Such cases cannot be treated by means of the classical perturbation theory, and a roundabout way must be sought. (If at least one of the two reasons mentioned above does not occur, the problem 
simplifies.) We take $t=|\mathbf{k}|$ as the perturbation parameter; then we must make our constructions and estimates uniform in $\boldsymbol{\theta}=t^{-1} \mathbf{k}$.

We start with an abstract operator theory method (see Chapter 1), distinguishing the case where the family in question admits an analytic factorization. This additional structure allows us to advance unexpectedly far in abstract terms. Often, the operators occurring in applications admit the required factorization from the outset. In some other cases, such factorization can be introduced forcedly. In the absence of the required factorization, the study of the threshold properties becomes much more difficult; the clear understanding of this is useful.

Keeping in mind applications to (vector) second order DO's, in the abstract method we restrict ourselves to the quadratic dependence on the parameter. The crucial point for us is to distinguish and study the notion of the spectral germ of an operator family at $t=0$ (see Chapter 1, 1 ). In general, we believe that an effect should be classified as threshold if it can be described in terms of the corresponding spectral germ. The abstract form of the threshold effects is considered in Chapter $1, \S 5$. For instance, estimate (5.10) gives an abstract answer to the question about the behavior of periodic DO's in the small period limit. To a great extent, the present paper is devoted to realization of this approach, as applied to DO's. However, the specific properties of DO's are not employed much.

0.3. The threshold effects are responsible for the emergence of the so-called effective characteristics. We believe that the mechanism of their appearance is as follows. Since a threshold effect is determined by the spectral germ only, in the description of this effect the initial periodic DO can be replaced by any other periodic DO with the same spectral germ. Among these "equivalent" DO's, some simple operators may occur; often, these are DO's with constant coefficients. That is why the notions of effective mass, effective Hamiltonian, etc., arise in quantum mechanics problems, and the notions of effective (homogenized) medium and homogenized DO arise in problems concerning periodic structures with vanishing period. The advantages of description in terms of effective characteristics are obvious. Usually, the idea itself of the existence of effective characteristics has physical origin. At the same time, when using the effective characteristics, one should remember that, in fact, they only keep information about the spectral germ, and, moreover, in a disguised form.

0.4. In Chapter 2, we distinguish a rather wide class of elliptic periodic second order DO's that act in $L_{2}\left(\mathbb{R}^{d} ; \mathbb{C}^{n}\right)$. This class includes a number of operators of mathematical physics, though it does not cover all needs of applications. Under the Gelfand transformation, each operator $\mathcal{A}$ of this class generates an operator family $A(t, \boldsymbol{\theta}), t \boldsymbol{\theta}=\mathbf{k}$, in $L_{2}\left(\Omega ; \mathbb{C}^{n}\right)$, which admits a factorization analytic in $t=|\mathbf{k}|$. Now, the spectral germ $S(\boldsymbol{\theta})$ for $A(t, \boldsymbol{\theta})$ at $t=0$ depends on the parameter $\boldsymbol{\theta}$; this is essential for further considerations.

In Chapter 3, on the basis of the general results of Chapter 1 , for each operator $\mathcal{A}$ we introduce the effective characteristics. These are defined directly via the corresponding germ $S(\boldsymbol{\theta})$. In Chapter 4 we show that these characteristics are responsible for the homogenization procedure for DO in the small period limit.

0.5. At present, the study of periodic problems with rapidly varying medium parameters (with small period) is a broad field of theoretical and applied science. There are various methods specific for this field, and a lot of significant results. Limit procedures for boundary value problems in bounded domains have been studied; methods for constructing full asymptotic expansions (with respect to the small period) have been developed; homogenization procedures have been analyzed for nonselfadjoint operators, nonstationary operators, and nonlinear problems. Many surveys and monographs are devoted to 
these topics. For us, acquaintance with the remarkable books [BaPa, BeLP, ZhKO] turned out to be especially useful. We are far from the idea to revise all this large area by our methods. At the same time, we would like to draw the reader's attention to the following.

0.6. In homogenization theory, finding the effective characteristics is the initial problem, which usually is solved by direct methods. Even in the cases where the Floquet-Bloch decomposition and the analytic perturbation theory (usually, for a simple eigenvalue) are employed for this purpose, these methods are viewed as purely technical and are not related to the general notion of a threshold effect. The essence of our approach is in distinguishing the notion of the spectral germ at $\lambda=0$. The germ directly determines the effective characteristics, which then arise inevitably in any specific threshold effect. The homogenization in the small period limit is one of such effects; another corresponds to the problem about the negative discrete spectrum mentioned before. In Chapters $5-7$, we use traditional operators of mathematical physics to illustrate our unified approach. Many facts mentioned there are new for the corresponding specific operators, though they follow from the general pattern of Chapters 1-4 almost immediately. Apparently, the possibilities offered by our approach are not exhausted by the applications presented.

0.7. Formally speaking, the present text is written as a survey. This means more care about the reader's interests than it is customary in research papers. In this connection, we note that the content of this Introduction is supplemented in Subsections 4.1 and 7.1, in comments on Chapters 4-7, and in concluding remarks. Most of references and comparisons can be found there.

We clearly understand that the present text is in no way a survey on homogenization theory. This would be impossible not only for reasons of volume, but also because the authors are not experts in that theory. However, having been pondering for a number of years over spectral phenomena near thresholds, we have realized that homogenization is one of the most pronounced threshold effects. Thus, the paper is a step-by-step presentation of our point of view on homogenization as a threshold effect. Of course, other authors also employed this viewpoint; we mention, e.g., the articles [Zh1] and [Se]. However, we develop the "threshold" approach more systematically, and we use operator theory as the basis for our considerations.

The paper $\mathrm{BSu} 2$ is the initial version of the present exposition. As compared to BSu2], we refined the abstract part, bridged a number of technical gaps, and extended the collection of applications. Primarily, the latter concerns Chapter 7. The organization of the paper is clear from the table of contents.

0.8. Notation. Let $\mathfrak{H}$ and $\mathfrak{G}$ be two separable Hilbert spaces. The symbols $(\cdot, \cdot)_{\mathfrak{H}}$ and $\|\cdot\|_{\mathfrak{H}}$ denote the inner product and the norm in $\mathfrak{H}$; the symbol $\|\cdot\|_{\mathfrak{H} \rightarrow \mathfrak{G}}$ stands for the norm of a bounded operator from $\mathfrak{H}$ to $\mathfrak{G}$. If $\mathfrak{H}=\mathfrak{G}$, we write only one index in the notation of the operator norm. Sometimes, we omit the indices if this does not lead to confusion. $I=I_{\mathfrak{H}}$ is the identity operator on $\mathfrak{H}$. If $\mathfrak{N}$ is a subspace in $\mathfrak{H}$, then $\mathfrak{N}^{\perp}:=\mathfrak{H} \ominus \mathfrak{N}$. If $P$ is the orthogonal projection of $\mathfrak{H}$ onto $\mathfrak{N}$, then $P^{\perp}$ is the orthogonal projection onto $\mathfrak{N}^{\perp}$. For a closed operator $T$ in $\mathfrak{H}, \sigma(T)$ denotes its spectrum, and $\rho(T)$ denotes the set of its regular points. The symbol $\langle\cdot, \cdot\rangle\rangle$ stands for the standard inner product in $\mathbb{C}^{n} ;|\cdot|$ denotes the norm of a vector in $\mathbb{C}^{n}$; the unit $(n \times n)$-matrix is denoted by $\mathbf{1}_{n}$. Next, we use the notation $\mathbf{x}=\left(x^{1}, \ldots, x^{d}\right) \in \mathbb{R}^{d}, i D_{j}=\partial_{j}=\partial / \partial x^{j}, j=1, \ldots, d$, $\nabla=\operatorname{grad}=\left(\partial_{1}, \ldots, \partial_{d}\right), \mathbf{D}=-i \nabla=\left(D_{1}, \ldots, D_{d}\right), \nabla^{*}=-$ div. The $L_{p}$-spaces of $\mathbb{C}^{n}$-valued functions in a domain $\Omega \subseteq \mathbb{R}^{d}$ are denoted by $L_{p}\left(\Omega ; \mathbb{C}^{n}\right), 1 \leq p \leq \infty$. The Sobolev classes of order $s$ with integrability index $p$ of $\mathbb{C}^{n}$-valued functions in a domain $\Omega \subseteq \mathbb{R}^{d}$ are denoted by $W_{p}^{s}\left(\Omega ; \mathbb{C}^{n}\right)$. For $p=2$ we denote this space by $H^{s}\left(\Omega ; \mathbb{C}^{n}\right)$, 
$s \in \mathbb{R}$. If $n=1$, we write simply $W_{p}^{s}(\Omega), H^{s}(\Omega)$. Within one chapter, we use two-digit numbers for subsections, statements, and formulas. When referring to other chapters, we use three-digit enumeration (where the first number stands for the chapter).

0.9. We are grateful to V. Ivriǔ, P. Kuchment, V. V. Zhikov, and A. L. Pyatnitskiŭ for fruitful scientific contacts that stimulated our interest in the topic of the present paper. Especially, we would like to mention the survey $[\mathrm{Ku}]$ by P. Kuchment on the theory of photonic crystals.

The authors thank R. G. Shterenberg who has read the manuscript and found some inaccuracies.

The authors are grateful to the International Mittag-Leffler Institute in Stockholm and to the head of the program Professor A. Laptev for hospitality. Our stay in the Institute in the fall of 2002 contributed to completing this work.

\section{Chapter 1. Operator Families admitting FaCtorization}

The material of this chapter concerns the spectral perturbation theory for selfadjoint operator families. Our goal is to specify the case where the positive operator family in question admits factorization. Our considerations are adapted to the study of the threshold effects near the lower edge of the spectrum. The resulting estimates are efficient for applications, and the constants in estimates are well controlled.

\section{§1. Quadratic PENCILS OF THE FORM $X(t)^{*} X(t)$}

1.1. Pencils of the form $X(t)$ and $X(t)^{*} X(t)$. Let $\mathfrak{H}$ and $\mathfrak{H}_{*}$ be complex separable Hilbert spaces. Suppose that $X_{0}: \mathfrak{H} \rightarrow \mathfrak{H}_{*}$ is a densely defined and closed operator and that $X_{1}: \mathfrak{H} \rightarrow \mathfrak{H}_{*}$ is a bounded operator. Then the operator (the linear operator pencil)

$$
X(t)=X_{0}+t X_{1}, \quad t \in \mathbb{R},
$$

is closed on the domain $\operatorname{Dom} X(t):=\operatorname{Dom} X_{0}$. We have $X(t)^{*}=X_{0}^{*}+t X_{1}^{*}$ on the domain $\operatorname{Dom} X_{0}^{*}$ (which is dense in $\mathfrak{H}_{*}$ ). The selfadjoint positive operator family

$$
A(t):=X(t)^{*} X(t)
$$

in $\mathfrak{H}$ is our main object. The operator (1.2) is generated by the closed quadratic form $\|X(t) u\|_{\mathfrak{H}_{*}}^{2}, u \in \operatorname{Dom} X_{0}$. For the operator $A_{0}:=A(0)=X_{0}^{*} X_{0}$, we put

$$
\mathfrak{N}:=\operatorname{Ker} A_{0}=\operatorname{Ker} X_{0} .
$$

Throughout, we assume that the following condition is fulfilled.

Condition 1.1. The point $\lambda=0$ is an isolated point of the spectrum of $A_{0}$, and

$$
(0<) n:=\operatorname{dim} \mathfrak{N}<\infty \text {. }
$$

We denote by $F(t, s)$ the spectral projection of the operator $A(t)$ for a closed interval $[0, s]$, and put $\mathfrak{F}(t, s):=F(t, s) \mathfrak{H}$. We fix a number $\delta>0$ such that $8 \delta<d^{0}$, where $d^{0}$ is the distance from the point $\lambda=0$ to the rest of the spectrum of $A_{0}$. We often write $F(t)$ in place of $F(t, \delta)$ and $\mathfrak{F}(t)$ in place of $\mathfrak{F}(t, \delta)$. The following statement is an easy consequence of the spectral theorem.

Proposition 1.2. We have

$$
F(t, \delta)=F(t, 3 \delta), \quad \operatorname{rank} F(t, \delta)=n, \quad|t| \leq t^{0}=t^{0}(\delta):=\delta^{1 / 2}\left\|X_{1}\right\|^{-1} .
$$


Proof. Assume that $\operatorname{rank} F(t, \delta)<n$. Then there exists an element $f \in \mathfrak{N}$ such that $\|f\|=1$ and $f \perp \mathfrak{F}(t, \delta)$. Hence, $\|X(t) f\|^{2}>\delta$. On the other hand, $\|X(t) f\|^{2}=$ $\left\|X_{0} f+t X_{1} f\right\|^{2} \leq t^{2}\left\|X_{1}\right\|^{2} \leq \delta$. This contradiction shows that

$$
\operatorname{rank} F(t, \delta) \geq n \text {. }
$$

Now, assume that $\operatorname{rank} F(t, 3 \delta)>n$. Then there exists $f \in \mathfrak{F}(t, 3 \delta)$ such that $\|f\|=1$ and $f \perp \mathfrak{N}$. Therefore, $\left\|X_{0} f\right\|^{2} \geq d^{0}>8 \delta$. But

$$
\left\|X_{0} f\right\|^{2} \leq 2\|X(t) f\|^{2}+2 t^{2}\left\|X_{1}\right\|^{2} \leq 6 \delta+2 \delta=8 \delta,
$$

a contradiction. Consequently,

$$
\operatorname{rank} F(t, 3 \delta) \leq n .
$$

Comparing (1.4) and (1.5), we see that $\operatorname{rank} F(t, \delta)=\operatorname{rank} F(t, 3 \delta)=n$, which is equivalent to (1.3).

Together with (1.2), it is convenient to consider the selfadjoint operator family

$$
A_{*}(t):=X(t) X(t)^{*}
$$

in $\mathfrak{H}_{*}$. We put $A_{* 0}:=X_{0} X_{0}^{*}$,

$$
\mathfrak{N}_{*}:=\operatorname{Ker} A_{* 0}=\operatorname{Ker} X_{0}^{*}, \quad n_{*}:=\operatorname{dim} \mathfrak{N}_{*} .
$$

The operators $A(t)$ and $A_{*}(t)$ have the same nonzero spectrum. In general, the numbers $n$ and $n_{*}$ are distinct. We assume that

$$
n \leq n_{*} \leq \infty .
$$

Let $P$ and $P_{*}$ denote the orthogonal projections onto $\mathfrak{N}$ and $\mathfrak{N}_{*}$, respectively.

1.2. The operator $R$. We introduce the notation $\mathcal{D}:=\operatorname{Dom} X_{0} \cap \mathfrak{N}^{\perp}$. Since the point $\lambda=0$ is isolated in $\sigma\left(A_{0}\right)$, the form $\left(X_{0} \varphi, X_{0} \zeta\right)_{\mathfrak{H}_{*}}, \varphi, \zeta \in \mathcal{D}$, determines an inner product in $\mathcal{D}$, converting $\mathcal{D}$ into a Hilbert space. Let $z \in \mathfrak{H}_{*}$. Suppose $\widehat{\varphi} \in \mathcal{D}$ satisfies the equation $X_{0}^{*}\left(X_{0} \widehat{\varphi}-z\right)=0$, which is understood in the weak sense. In other words, we look for an element $\widehat{\varphi} \in \mathcal{D}$ satisfying the identity

$$
\left(X_{0} \widehat{\varphi}, X_{0} \zeta\right)_{\mathfrak{H}_{*}}=\left(z, X_{0} \zeta\right)_{\mathfrak{H}_{*}}, \quad \zeta \in \mathcal{D}
$$

Since the right-hand side of (1.7) is an antilinear continuous functional of $\zeta \in \mathcal{D}$, there exists a unique solution $\widehat{\varphi}$. Moreover, $\left\|X_{0} \widehat{\varphi}\right\|_{\mathfrak{H}_{*}} \leq\|z\|_{\mathfrak{H}_{*}}$. Note also that $X_{0} \widehat{\varphi}-z \in \mathfrak{N}_{*}$.

Now, let

$$
\omega \in \mathfrak{N}, \quad z:=-X_{1} \omega .
$$

We denote by $\widehat{\varphi}(\omega)$ the element $\widehat{\varphi}$ satisfying (1.7) with this $z$ and put

$$
\omega_{*}:=X_{0} \widehat{\varphi}(\omega)+X_{1} \omega \in \mathfrak{N}_{*} .
$$

In accordance with (1.7)-(1.9), we introduce the linear operator $R$ that takes $\omega$ to $\omega_{*}$ :

$$
R: \mathfrak{N} \rightarrow \mathfrak{N}_{*}, \quad R \omega=\omega_{*} .
$$

Clearly, $\omega_{*} \in \mathfrak{N}_{*}$ and $X_{0} \widehat{\varphi}(\omega) \in \operatorname{Ran} X_{0} \subset \mathfrak{N}_{*}^{\perp}$. If follows that $\omega_{*}=P_{*} X_{1} \omega$. This yields another representation for $R$ :

$$
R=\left.P_{*} X_{1}\right|_{\mathfrak{N}},
$$

which is equivalent to

$$
P_{*} X_{1} P=R \oplus \mathbb{O}
$$

(we extend $R$ to $\mathfrak{N}^{\perp}$ by zero). The continuous operator $R_{*}: \mathfrak{N}_{*} \rightarrow \mathfrak{N}$ is defined in a similar way. Then

$$
R_{*}=\left.P X_{1}^{*}\right|_{\mathfrak{N}_{*}}, \quad P X_{1}^{*} P_{*}=R_{*} \oplus \mathbb{O} .
$$


Relations (1.11)-(1.13) imply that

$$
R_{*}=R^{*} .
$$

1.3. The spectral germ $S$ of the family $A(t)$ at $t=0$.

Definition 1.3. The selfadjoint operator

$$
S:=R^{*} R: \mathfrak{N} \rightarrow \mathfrak{N}
$$

is called the spectral germ of the operator family (1.2) at $t=0$.

From (1.12)-(1.14) it follows that

$$
S=\left.P X_{1}^{*} P_{*} X_{1}\right|_{\mathfrak{N}}, \quad S \oplus \mathbb{O}=P X_{1}^{*} P_{*} X_{1} P .
$$

Observe also the identity

$$
(S \zeta, \zeta)_{\mathfrak{H}}=\|R \zeta\|_{\mathfrak{H}_{*}}^{2}=\left\|P_{*} X_{1} \zeta\right\|_{\mathfrak{H}_{*}}^{2}, \quad \zeta \in \mathfrak{N} .
$$

For the family (1.6), the role of the spectral germ is played by $S_{*}:=R R^{*}: \mathfrak{N}_{*} \rightarrow \mathfrak{N}_{*}$. The part of the operator $S$ acting in $(\operatorname{Ker} S)^{\perp}$ is unitarily equivalent to the part of $S_{*}$ acting in $\left(\operatorname{Ker} S_{*}\right)^{\perp}$. Below (cf. Subsection 1.6), we shall clarify the role of the operator $S$. In particular, it will be shown that $S$ is independent of the choice of a factorization as in (1.2) for $A(t)$.

Definition 1.4. The spectral germ $S$ of the operator family (1.2) at $t=0$ is called nondegenerate if $\operatorname{Ker} S=\{0\}$.

Obviously, the nondegeneracy of $S$ is equivalent to the condition $\operatorname{Ker} R=\{0\}$, or, equivalently, to the condition $\operatorname{rank} R=n$.

Let $\widetilde{A}(t)=\widetilde{X}(t)^{*} \widetilde{X}(t)$ be yet another operator family in $\mathfrak{H}$ subject to the same conditions (see Subsection 1.1) as $A(t)$. (The space $\widetilde{\mathfrak{H}}_{*}$ may be different from $\mathfrak{H}_{*}$.) Let $\widetilde{\mathfrak{N}}:=\operatorname{Ker} \widetilde{A}(0)$, and let $\widetilde{S}: \widetilde{\mathfrak{N}} \rightarrow \widetilde{\mathfrak{N}}$ be the spectral germ of the family $\widetilde{A}(t)$ at $t=0$.

Definition 1.5. The operator families $A(t)$ and $\widetilde{A}(t)$ are said to be threshold equivalent if $\mathfrak{N}=\widetilde{\mathfrak{N}}$ and $S=\widetilde{S}$.

This relation is an equivalence on the set of operator families of the form (1.2).

1.4. Estimates for the operator $S$. Upper estimates. Let $\mathfrak{N}_{*} \subset \mathfrak{G}_{*} \subset \mathfrak{H}_{*}$, where $\mathfrak{G}_{*}$ is a subspace in $\mathfrak{H}_{*}$, and let $\Pi_{*}$ be the orthogonal projection onto $\mathfrak{G}_{*}$. Obviously, (1.16) implies the inequality

$$
S \oplus \mathbb{O} \leq P X_{1}^{*} \Pi_{*} X_{1} P,
$$

or, equivalently,

$$
(S \zeta, \zeta)_{\mathfrak{H}} \leq\left\|\Pi_{*} X_{1} \zeta\right\|_{\mathfrak{H}_{*}}^{2}, \quad \zeta \in \mathfrak{N}
$$

(cf. also (1.17)). In particular, if $\mathfrak{G}_{*}=\mathfrak{H}_{*}$, we obtain

$$
(S \zeta, \zeta)_{\mathfrak{H}} \leq\left\|X_{1} \zeta\right\|_{\mathfrak{H}_{*}}^{2}, \quad \zeta \in \mathfrak{N}
$$

Finally, (1.19) (or (1.16)) implies the estimate $\|S\| \leq\left\|X_{1}\right\|^{2}$. Inequality (1.18) offers an opportunity of refining estimates for the operator $S$ on the basis of the Ritz method.

Lower estimates for $S$. Let $\mathfrak{G}_{*}^{\prime}$ be a subspace in $\mathfrak{N}_{*}$, and let $\Pi_{*}^{\prime}$ be the orthogonal projection onto $\mathfrak{G}_{*}^{\prime}$. Then $S \oplus \mathbb{O} \geq P X_{1}^{*} \Pi_{*}^{\prime} X_{1} P$, or

$$
(S \zeta, \zeta)_{\mathfrak{H}} \geq\left\|\Pi_{*}^{\prime} X_{1} \zeta\right\|_{\mathfrak{H}_{*}}^{2}, \quad \zeta \in \mathfrak{N}
$$


1.5. The case where $X(t)=\widehat{X}(t) M$. Let $\widehat{\mathfrak{H}}$ be yet another Hilbert space, and let $\widehat{X}(t)=\widehat{X}_{0}+t \widehat{X}_{1}: \widehat{\mathfrak{H}} \rightarrow \mathfrak{H}_{*}$ be a family of the form (1.1) that satisfies the assumptions of Subsection 1.1. We emphasize that the space $\mathfrak{H}_{*}$ is the same as before. Suppose that $M: \mathfrak{H} \rightarrow \widehat{\mathfrak{H}}$ is an isomorphism. Suppose $M \operatorname{Dom} X_{0}=\operatorname{Dom} \widehat{X}_{0}, X(t)=\widehat{X}(t) M: \mathfrak{H} \rightarrow$ $\mathfrak{H}_{*}, X_{0}=\widehat{X}_{0} M, X_{1}=\widehat{X}_{1} M$, and

$$
A(t)=M^{*} \widehat{A}(t) M .
$$

In what follows, all the objects related to $\widehat{X}(t)$ are marked by "^^". Observe that

$$
\widehat{\mathfrak{N}}=M \mathfrak{N}, \quad \widehat{n}=n, \quad \widehat{\mathfrak{N}}_{*}=\mathfrak{N}_{*}, \quad \widehat{n}_{*}=n_{*}, \quad \widehat{P}_{*}=P_{*} .
$$

Moreover, $R=\left.P_{*} X_{1}\right|_{\mathfrak{N}}=\left.\widehat{P}_{*} \widehat{X}_{1} M\right|_{\mathfrak{N}}$, i.e.,

$$
R=\left.\widehat{R} M\right|_{\mathfrak{N}} .
$$

In accordance with (1.17) and (1.21), for $\zeta \in \mathfrak{N}$ and $\widehat{\zeta}=M \zeta(\in \widehat{\mathfrak{N}})$ we have

$$
(S \zeta, \zeta)_{\mathfrak{H}}=\|R \zeta\|_{\mathfrak{H}_{*}}^{2}=\|\widehat{R} \widehat{\zeta}\|_{\mathfrak{H}_{*}}^{2}=(\widehat{S} \widehat{\zeta}, \widehat{\zeta})_{\widehat{\mathfrak{H}}}=\left(M^{*} \widehat{S} M \zeta, \zeta\right)_{\mathfrak{H}}
$$

Consequently,

$$
\begin{array}{rlrl}
S & =\left.P M^{*} \widehat{S} M\right|_{\mathfrak{N}}, & & S \oplus \mathbb{O}=P M^{*} \widehat{S} M P, \\
\widehat{S} & =\left.\widehat{P}\left(M^{*}\right)^{-1} S M^{-1}\right|_{\widehat{\mathfrak{N}}}, & \widehat{S} \oplus \mathbb{O}=\widehat{P}\left(M^{*}\right)^{-1} S M^{-1} \widehat{P} .
\end{array}
$$

Formulas (1.24) follow from (1.23) by interchanging the roles of $X(t)$ and $\widehat{X}(t)$. Relation (1.21) implies that $\operatorname{rank} R=\operatorname{rank} \widehat{R}$; therefore, $S$ and $\widehat{S}$ are nondegenerate or degenerate simultaneously. By (1.22), we have

$$
\frac{(S \zeta, \zeta)_{\mathfrak{H}}}{\|\zeta\|_{\mathfrak{H}}^{2}}=\frac{(\widehat{S} \widehat{\zeta}, \widehat{\zeta})_{\widehat{\mathfrak{H}}}}{\|\widehat{\zeta}\|_{\widehat{\mathfrak{H}}}^{2}} \frac{\|M \zeta\|_{\widehat{\mathfrak{H}}}^{2}}{\|\zeta\|_{\mathfrak{H}}^{2}} \leq\|M\|^{2} \frac{(\widehat{S} \widehat{\zeta}, \widehat{\zeta})_{\widehat{\mathfrak{H}}}}{\|\widehat{\zeta}\|_{\widehat{\mathfrak{H}}}^{2}}
$$

and, similarly, $(\widehat{S} \widehat{\zeta}, \widehat{\zeta})_{\hat{\mathfrak{H}}}\|\widehat{\zeta}\|_{\widehat{\mathfrak{H}}}^{-2} \leq\left\|M^{-1}\right\|^{2}(S \zeta, \zeta)_{\mathfrak{H}}\|\zeta\|_{\mathfrak{H}}^{-2}$. Thus, the eigenvalues $\gamma_{l}$ of $S$ and the eigenvalues $\widehat{\gamma}_{l}$ of $\widehat{S}$ satisfy

$$
\left\|M^{-1}\right\|^{-2} \widehat{\gamma}_{l} \leq \gamma_{l} \leq\|M\|^{2} \widehat{\gamma}_{l}, \quad l=1, \ldots, n .
$$

Now, let $n=1$. Then (1.25) implies the following relation for $\gamma=\gamma_{1}, \widehat{\gamma}=\widehat{\gamma}_{1}$ :

$$
\gamma=\widehat{\gamma}\|M \zeta\|_{\mathfrak{H}}^{2}\|\zeta\|_{\mathfrak{H}}^{-2}, \quad \zeta \in \mathfrak{N} .
$$

1.6. The eigenvalue problem for the operator $S$. The general analytic perturbation theory (see [Ka]) says that, for $|t| \leq t^{0}$, there exist real-analytic functions $\lambda_{l}(t)$ (branches of eigenvalues) and real-analytic $\mathfrak{H}$-valued functions $\varphi_{l}(t)$ (branches of eigenvectors) such that

$$
A(t) \varphi_{l}(t)=\lambda_{l}(t) \varphi_{l}(t), \quad l=1, \ldots, n, \quad|t| \leq t^{0}=t^{0}(\delta),
$$

and the $\varphi_{l}(t), l=1, \ldots, n$, form an orthonormal basis in $\mathfrak{F}(t)$. Moreover, for sufficiently small $t_{*}$, we have the convergent power series expansions

$$
\begin{array}{lll}
\lambda_{l}(t)=\gamma_{l} t^{2}+\cdots, & & \gamma_{l} \geq 0, \quad l=1, \ldots, n, \quad|t| \leq t_{*} \leq t^{0}, \\
\varphi_{l}(t)=\omega_{l}+t \varphi_{l}^{(1)}+t^{2} \varphi_{l}^{(2)}+\cdots, & & l=1, \ldots, n,|t| \leq t_{*} \leq t^{0} .
\end{array}
$$

The elements $\omega_{l}:=\varphi_{l}(0), l=1, \ldots, n$, form an orthonormal basis in $\mathfrak{N}$, and $P=$ $\sum_{l=1}^{n}\left(\cdot, \omega_{l}\right) \omega_{l}$. Relations (1.27) are equivalent to

$$
\left(X(t) \varphi_{l}(t), X(t) \zeta\right)_{\mathfrak{H}_{*}}=\lambda_{l}(t)\left(\varphi_{l}(t), \zeta\right)_{\mathfrak{H}}, \quad \zeta \in \operatorname{Dom} X_{0}
$$


The numbers $\gamma_{l}$ and the elements $\omega_{l}, \varphi_{l}^{(1)}, \varphi_{l}^{(2)}, \ldots$, can be found from (1.30) and from the normalization condition $\left\|\varphi_{l}(t)\right\|_{\mathfrak{H}}^{2}=1$, by comparing the coefficients of the same powers of $t$. Comparing the first order terms, we obtain (see (1.7)-(1.9))

$$
\varphi_{l}^{(1)}-\widehat{\varphi}\left(\omega_{l}\right) \in \mathfrak{N}, \quad l=1, \ldots, n .
$$

Comparing the terms with $t^{2}$, we see that

$$
\left(X_{0} \varphi_{l}^{(1)}+X_{1} \omega_{l}, X_{1} \zeta\right)_{\mathfrak{H}_{*}}+\left(X_{0} \varphi_{l}^{(2)}+X_{1} \varphi_{l}^{(1)}, X_{0} \zeta\right)_{\mathfrak{H}_{*}}=\gamma_{l}\left(\omega_{l}, \zeta\right)_{\mathfrak{H}}, \quad \zeta \in \operatorname{Dom} X_{0}
$$

By (1.9), (1.10), and (1.31), we have $X_{0} \varphi_{l}^{(1)}+X_{1} \omega_{l}=R \omega_{l}$. For $\zeta \in \mathfrak{N}$, relation (1.32) takes the form

$$
\left(R \omega_{l}, X_{1} \zeta\right)_{\mathfrak{H}_{*}}=\gamma_{l}\left(\omega_{l}, \zeta\right)_{\mathfrak{H}}, \quad \zeta \in \mathfrak{N}
$$

By (1.11) and (1.16),

$$
\left(R \omega_{l}, X_{1} \zeta\right)_{\mathfrak{H}_{*}}=\left(P X_{1}^{*} R \omega_{l}, \zeta\right)_{\mathfrak{H}}=\left(S \omega_{l}, \zeta\right)_{\mathfrak{H}}
$$

Thus, (1.33) yields the following statement.

Proposition 1.6. The numbers $\gamma_{l}$ and the elements $\omega_{l}$ defined by (1.27)-(1.29) are eigenvalues and eigenvectors of the operator $S$ :

$$
S \omega_{l}=\gamma_{l} \omega_{l}, \quad l=1, \ldots, n .
$$

This clarifies the meaning of the eigenvalue problem for $S$. Relations (1.34) show that

$$
S P=\sum_{l=1}^{n} \gamma_{l}\left(\cdot, \omega_{l}\right)_{\mathfrak{H}} \omega_{l} .
$$

The series (1.28) and (1.29) are determined by the family $A(t)$ directly, independently of factorization (1.2). This shows that $S$ does not depend on the choice of factorization. At the same time, relation (1.15) can be interpreted as the fact that the factorization chosen for $A(t)$ is inherited by $S$. The nondegeneracy of $S$ is equivalent to the inequalities

$$
\gamma_{l} \geq c_{*}>0, \quad l=1, \ldots, n,
$$

for the numbers $\gamma_{l}$ defined by (1.28).

If all the eigenvalues $\gamma_{l}$ are simple, then from (1.34) we can find the initial elements $\omega_{l}$ in (1.29) (up to a phase factor). If multiple eigenvalues occur among the $\gamma_{l}$, then, in general, the knowledge of $S$ does not suffice for this purpose.

1.7. Operator-valued functions $F(t)$ and $A(t) F(t)$. For what follows, it is important to find "good" approximations for the operator-valued functions $F(t)$ and $A(t) F(t)$. Both functions are real-analytic for $|t| \leq t^{0}(\delta)$. From (1.29) and Proposition 1.2 it follows that, for sufficiently small $t_{*} \leq t^{0}(\delta)$, we have

$$
F(t, \delta)=F(t)=P+t F_{1}+\cdots, \quad|t| \leq t_{*},
$$

where $F_{1}=\check{F}_{1}+\check{F}_{1}^{*}, \check{F}_{1}=\sum_{l=1}^{n}\left(\cdot, \omega_{l}\right)_{\mathfrak{H}} \varphi_{l}^{(1)}$. Next, by (1.27) and Proposition 1.2,

$$
A(t) F(t)=\sum_{l=1}^{n} \lambda_{l}(t)\left(\cdot, \varphi_{l}(t)\right)_{\mathfrak{H}} \varphi_{l}(t), \quad|t| \leq t^{0}(\delta) .
$$

Combining this with (1.28), (1.29), and (1.35), we obtain

$$
A(t) F(t)=t^{2} S P+\cdots, \quad|t| \leq t_{*} .
$$

The significance of (1.38) is in the fact that $S$ admits the representations (1.15) and (1.16), which do not require the knowledge of the eigenvectors $\omega_{1}, \ldots, \omega_{n}$.

However, the power series expansions (1.37) and (1.38) are not quite suitable for our purposes. We need only estimates of $F(t)-P$ and $A(t) F(t)-t^{2} S P$, but on the 
wider interval $|t| \leq t^{0}(\delta)$, and with constants controlled explicitly. Such estimates can be obtained by integrating the difference of the resolvents for $A(t)$ and $A_{0}$ over an appropriate contour $\Gamma$. However, the difficulty is that the ordinary resolvent identity $(A(t)-z I)^{-1}-\left(A_{0}-z I\right)^{-1}=\left(A_{0}-z I\right)^{-1}\left(A_{0}-A(t)\right)(A(t)-z I)^{-1}$ is not applicable under our assumptions. Indeed, in general, the difference $A(t)-A_{0}$ makes no sense. The only remaining possibility is to use the version of the resolvent identity for operators whose quadratic forms have the same domain. (In our case, the domain of the quadratic form coincides with Dom $X(t)=\operatorname{Dom} X_{0}$.) Since the corresponding relations are not of common use, in the next $\S 2$ we present the necessary auxiliary material.

1.8. Families with block structure. Families of the form

$$
\mathbf{X}(t)=\left(\begin{array}{cc}
0 & X(t)^{*} \\
X(t) & 0
\end{array}\right)
$$

act in the space

$$
\mathcal{H}=\mathfrak{H} \oplus \mathfrak{H}_{*} .
$$

It is natural to assume that $n=n_{*}$. Let $\mathbf{w}=\operatorname{col}(u, v), \mathbf{s}=\operatorname{col}(q, r) \in \mathcal{H}$, and let

$$
(\mathbf{X}(t)-i \varkappa I) \mathbf{w}=\mathbf{s}, \quad \varkappa>0 \text {. }
$$

We write the solution $\mathbf{w}$ of equation (1.39) as

$$
\mathbf{w}=\mathbf{w}_{q}+\mathbf{w}_{r},
$$

where $\mathbf{w}_{q}$ is the solution of (1.39) with $r=0$, and $\mathbf{w}_{r}$ is the solution of (1.39) with $q=0$. We have $\mathbf{w}_{q}=\operatorname{col}\left(u_{q}, v_{q}\right), \mathbf{w}_{r}=\operatorname{col}\left(u_{r}, v_{r}\right)$, and

$$
\begin{aligned}
& X(t)^{*} v_{q}-i \varkappa u_{q}=q, \quad X(t) u_{q}-i \varkappa v_{q}=0, \\
& X(t)^{*} v_{r}-i \varkappa u_{r}=0, \quad X(t) u_{r}-i \varkappa v_{r}=r .
\end{aligned}
$$

From (1.40) and (1.41) it follows that

$$
\begin{array}{ll}
u_{q}=i \varkappa\left(A(t)+\varkappa^{2} I\right)^{-1} q, & v_{q}=\left(A_{*}(t)+\varkappa^{2} I\right)^{-1} X(t) q, \\
u_{r}=\left(A(t)+\varkappa^{2} I\right)^{-1} X(t)^{*} r, & v_{r}=i \varkappa\left(A_{*}(t)+\varkappa^{2} I\right)^{-1} r .
\end{array}
$$

The functions $v_{q}$ and $u_{r}$ admit also other representations:

$$
v_{q}=X(t)\left(A(t)+\varkappa^{2} I\right)^{-1} q, \quad u_{r}=X(t)^{*}\left(A_{*}(t)+\varkappa^{2} I\right)^{-1} r .
$$

Observe that the expressions for $u_{q}$ and $v_{r}$ are simpler than those for $v_{q}$ and $u_{r}$.

\section{§2. Auxiliary material}

2.1. The resolvent identity. Let $\mathfrak{H}$ be a Hilbert space. The symbols $(\cdot, \cdot)$ and $\|\cdot\|$ stand for the inner product and the norm in this space. Let $a$ and $b$ be two sesquilinear nonnegative closed forms in $\mathfrak{H}$. Suppose that

$$
\operatorname{Dom} a=\operatorname{Dom} b=: \mathfrak{d}
$$

and that $\mathfrak{d}$ is dense in $\mathfrak{H}$. By $A$ (respectively, $B$ ) we denote the selfadjoint operator in $\mathfrak{H}$ generated by the form $a$ (respectively, $b$ ). We put

$$
a_{\gamma}[u, v]=a[u, v]+\gamma(u, v), \quad \gamma>0 .
$$

The notation $b_{\gamma}$ has a similar meaning. The linear set $\mathfrak{d}$ is a complete Hilbert space $\mathfrak{d}\left(a_{\gamma}\right)$ with respect to the inner product $(2.2)$. We denote the norm in $\mathfrak{d}\left(a_{\gamma}\right)$ by $\|\cdot\|_{\mathfrak{d}}$. Obviously,

$$
\|u\| \leq \gamma^{-1 / 2}\|u\|_{\mathfrak{o}}
$$


By (2.1), the form $b_{\gamma}$ is continuous on $\mathfrak{d}\left(a_{\gamma}\right)$, and in this space it generates a metric equivalent to the standard one. We put

$$
\alpha^{2}=\sup _{0 \neq f \in \mathfrak{d}} \frac{a_{\gamma}[f, f]}{b_{\gamma}[f, f]} .
$$

Obviously, the real-valued form $t=b-a$ is $\left(a_{\gamma}\right)$-continuous; therefore, it generates a continuous selfadjoint operator $T_{\gamma}$ in $\mathfrak{d}\left(a_{\gamma}\right)$. Thus,

$$
\begin{aligned}
t[u, v] & =a_{\gamma}\left[T_{\gamma} u, v\right], \quad u, v \in \mathfrak{d}, \\
\left\|T_{\gamma}\right\|_{\mathfrak{d}} & =\sup _{\substack{0 \neq u \in \mathfrak{d} \\
0 .}} \frac{|t[u, u]|}{a_{\gamma}[u, u]} .
\end{aligned}
$$

The operator $T_{\gamma}$ can be viewed as an operator from $\mathfrak{d}\left(a_{\gamma}\right)$ to $\mathfrak{H}$. Then, by (2.3),

$$
\left\|T_{\gamma}\right\|_{\mathfrak{o} \rightarrow \mathfrak{H}} \leq \gamma^{-1 / 2}\left\|T_{\gamma}\right\|_{\mathfrak{o}}
$$

Consider the equations

$$
(A+\gamma I) x=f, \quad(B+\gamma I) y=f,
$$

which are equivalent to the relations

$$
a_{\gamma}[x, v]=(f, v), \quad b_{\gamma}[y, v]=(f, v), \quad v \in \mathfrak{d} .
$$

From (2.5) it follows that

$$
b_{\gamma}[y, v]=a_{\gamma}[y, v]+t[y, v]=a_{\gamma}\left[\left(I+T_{\gamma}\right) y, v\right], \quad v \in \mathfrak{d},
$$

whence $x=y+T_{\gamma} y$. Thus,

$$
(B+\gamma I)^{-1}-(A+\gamma I)^{-1}=-T_{\gamma}(B+\gamma I)^{-1} .
$$

Let $R_{z}(A)$ and $R_{z}(B)$ denote the corresponding resolvents. Using the Hilbert identity $R_{z}(B)-R_{-\gamma}(B)=(z+\gamma) R_{-\gamma}(B) R_{z}(B)$ and the same identity for $R_{z}(A)$, we see that, by $(2.8)$,

$$
R_{z}(B)-R_{z}(A)=-T_{\gamma} R_{-\gamma}(B)\left(I+(z+\gamma) R_{z}(B)\right)+(z+\gamma) R_{-\gamma}(A)\left(R_{z}(B)-R_{z}(A)\right) .
$$

We introduce the notation

$$
\Omega_{z}(A):=I+(z+\gamma) R_{z}(A)
$$

and a similar notation $\Omega_{z}(B)$ for $B$. Since $\left(I-(z+\gamma) R_{-\gamma}(A)\right)^{-1}=\Omega_{z}(A)$, identity (2.9) implies that

$$
R_{z}(B)-R_{z}(A)=-\Omega_{z}(A) T_{\gamma} R_{-\gamma}(B) \Omega_{z}(B), \quad z \in \rho(A) \cap \rho(B) .
$$

Next, since

$$
R_{z}(B)=R_{-\gamma}(B) \Omega_{z}(B),
$$

from (2.11) we deduce that

$$
R_{z}(B)-R_{z}(A)=-\Omega_{z}(A) T_{\gamma} R_{z}(B), \quad z \in \rho(A) \cap \rho(B) .
$$

Relation (2.13) is an analog of the usual resolvent identity under the condition that (2.1) is fulfilled, but, possibly, Dom $A \neq \operatorname{Dom} B$. We keep calling (2.13) the resolvent identity. 
2.2. Estimates for operators of the form $L R_{z}(B)$ (where $L$ is a continuous operator in $\left.\mathfrak{d}\left(a_{\gamma}\right)\right)$. We write the identity

$$
L R_{-\gamma}(B)=L(A+\gamma I)^{-1 / 2}\left((A+\gamma I)^{1 / 2}(B+\gamma I)^{-1 / 2}\right)\left(R_{-\gamma}(B)\right)^{1 / 2} .
$$

Using (2.3) (see also (2.7)) and the fact that the mapping $(A+\gamma I)^{-1 / 2}: \mathfrak{H} \rightarrow \mathfrak{d}\left(a_{\gamma}\right)$ is an isometry, we obtain

$$
\left\|L(A+\gamma I)^{-1 / 2}\right\|_{\mathfrak{H}} \leq\left\|(A+\gamma I)^{-1 / 2}\right\|_{\mathfrak{H} \rightarrow \mathfrak{o}}\|L\|_{\mathfrak{o} \rightarrow \mathfrak{H}} \leq \gamma^{-1 / 2}\|L\|_{\mathfrak{o}} .
$$

Next, $\left\|R_{-\gamma}(B)\right\|_{\mathfrak{H}}^{1 / 2} \leq \gamma^{-1 / 2}$, and it is easily seen that $\left\|(A+\gamma I)^{1 / 2}(B+\gamma I)^{-1 / 2}\right\|_{\mathfrak{H}}=\alpha$, where $\alpha=\alpha(\gamma)$ is as in (2.4). Thus,

$$
\left\|L R_{-\gamma}(B)\right\|_{\mathfrak{H}} \leq \alpha \gamma^{-1}\|L\|_{\mathfrak{o}},
$$

and, by (2.12),

$$
\left\|L R_{z}(B)\right\|_{\mathfrak{H}} \leq \alpha \gamma^{-1}\|L\|_{\mathfrak{o}}\left\|\Omega_{z}(B)\right\|_{\mathfrak{H}}, \quad z \in \rho(B) .
$$

If we replace $B$ by $A$ in (2.15), then we must take $\alpha=1$, whence

$$
\left\|L R_{z}(A)\right\|_{\mathfrak{H}} \leq \gamma^{-1}\|L\|_{\mathfrak{o}}\left\|\Omega_{z}(A)\right\|_{\mathfrak{H}}, \quad z \in \rho(A) .
$$

We also need to estimate operators of the form $L_{1} \Omega_{z}(A) L_{2} R_{z}(B)$, where $L_{1}, L_{2}$ are continuous operators in $\mathfrak{d}\left(a_{\gamma}\right)$. By $(2.10)$,

$$
\left\|L_{1} \Omega_{z}(A) L_{2} R_{z}(B)\right\|_{\mathfrak{H}} \leq\left\|\left(L_{1} L_{2}\right) R_{z}(B)\right\|_{\mathfrak{H}}+|z+\gamma|\left\|L_{1} R_{z}(A)\right\|_{\mathfrak{H}}\left\|L_{2} R_{z}(B)\right\|_{\mathfrak{H}} .
$$

Inequalities (2.15) and (2.16) show that

$$
\left\|L_{1} \Omega_{z}(A) L_{2} R_{z}(B)\right\|_{\mathfrak{H}} \leq \alpha \gamma^{-1}\left\|\Omega_{z}(B)\right\|_{\mathfrak{H}}\left(1+|z+\gamma| \gamma^{-1}\left\|\Omega_{z}(A)\right\|_{\mathfrak{H}}\right)\left\|L_{1}\right\|_{\mathfrak{o}}\left\|L_{2}\right\|_{\mathfrak{o}}
$$

for $z \in \rho(A) \cap \rho(B)$.

\section{§3. Estimates For the Difference of Resolvents ON THE CONTOUR}

3.1. The contour $\Gamma$. We need to integrate the difference of resolvents $R_{z}(A(t))-$ $R_{z}(A(0))$ over the contour $\Gamma$ that envelopes the real interval $[0, \delta]$ equidistantly at the distance $\delta$. We recall that $\delta$ is a fixed number such that $8 \delta<d^{0}$, where $d^{0}$ is the distance between the nonzero part of the spectrum of $A_{0}$ and the point zero. The parameter $t$ is subject to the condition

$$
|t| \leq t^{0}=t^{0}(\delta)=\delta^{1 / 2}\left\|X_{1}\right\|^{-1},
$$

i.e., $t$ satisfies (1.3). Below, we shall write $R_{z}(t)$ in place of $R_{z}(A(t)), \Omega_{z}(t)$ in place of $\Omega_{z}(A(t))$, etc. Also, we omit the lower indices $\mathfrak{H}$ or $\mathfrak{H}_{*}$ in the notation of the norm and the inner product. By Proposition 1.2, if condition (3.1) is fulfilled, then the distance between $\Gamma$ and $\sigma(A(t))$ is at least $\delta$, whence

$$
\left\|R_{z}(t)\right\| \leq \delta^{-1}, \quad z \in \Gamma,|t| \leq t^{0}(\delta) .
$$

3.2. Incorporation into the pattern of $\S 2$. When using the results of $\S 2$, we assume that $\gamma=2 \delta$. To begin with, we mention an estimate for the operator-valued function $\Omega_{z}(t)=I+(z+2 \delta) R_{z}(t)$ of the form (2.10). By (3.2) and the inequality $|z| \leq 2 \delta$ for $z \in \Gamma$, we have

$$
\left\|\Omega_{z}(t)\right\| \leq 5, \quad z \in \Gamma, \quad|t| \leq t^{0}(\delta) .
$$

Now, the role of the forms $b$ and $a$ (see $\S 2$ ) is played by the forms $a(t)$ and $a(0)$, respectively:

$$
a(t)[u, u]=\|X(t) u\|^{2}, \quad a(0)[u, u]=\left\|X_{0} u\right\|^{2}, \quad u \in \operatorname{Dom} X_{0}=: \mathfrak{d} .
$$


First, we estimate the number $\alpha$ defined by (2.4). By (3.1),

$$
\begin{aligned}
\left\|X_{0} u\right\|^{2} & =\left\|\left(X(t)-t X_{1}\right) u\right\|^{2} \\
& \leq 2\|X(t) u\|^{2}+2 t^{2}\left\|X_{1}\right\|^{2}\|u\|^{2} \leq 2\left(\|X(t) u\|^{2}+\delta\|u\|^{2}\right), \quad u \in \mathfrak{d},
\end{aligned}
$$

which corresponds to the estimate

$$
\alpha^{2} \leq 2
$$

Now, the difference of the forms $a(t)-a(0)$ is equal to the form

$$
2 t \operatorname{Re}\left(X_{0} u, X_{1} u\right)+t^{2}\left\|X_{1} u\right\|^{2}, \quad u \in \mathfrak{d},
$$

which gives rise to an operator $T_{\gamma}$ in the space $\mathfrak{d}$ with the metric form $a_{\gamma}(0), \gamma=2 \delta$. From (3.5) it follows that the operator $T_{\gamma}=T_{\gamma}(t)$ can be represented as

$$
T_{\gamma}(t)=t T_{\gamma}^{(1)}+t^{2} T_{\gamma}^{(2)},
$$

where the operators $T_{\gamma}^{(1)}$ and $T_{\gamma}^{(2)}$ do not depend on $t$. We estimate their norms on $\mathfrak{d}$. Since

$$
2\left|\operatorname{Re}\left(X_{0} u, X_{1} u\right)\right| \leq \varkappa\left\|X_{0} u\right\|^{2}+\varkappa^{-1}\left\|X_{1}\right\|^{2}\|u\|^{2}, \quad \varkappa>0,
$$

for $\varkappa=(2 \delta)^{-1 / 2}\left\|X_{1}\right\|$ we obtain

$$
2\left|\operatorname{Re}\left(X_{0} u, X_{1} u\right)\right| \leq(2 \delta)^{-1 / 2}\left\|X_{1}\right\|\left(\left\|X_{0} u\right\|^{2}+2 \delta\|u\|^{2}\right),
$$

or, by $(2.6)$,

$$
\left\|T_{\gamma}^{(1)}\right\|_{\mathfrak{o}} \leq(2 \delta)^{-1 / 2}\left\|X_{1}\right\|
$$

Next, we have $\left\|X_{1} u\right\|^{2} \leq(2 \delta)^{-1}\left\|X_{1}\right\|^{2}\left(\left\|X_{0} u\right\|^{2}+2 \delta\|u\|^{2}\right)$, i.e.,

$$
\left\|T_{\gamma}^{(2)}\right\|_{\mathfrak{o}} \leq(2 \delta)^{-1}\left\|X_{1}\right\|^{2}
$$

Finally, we estimate the norm of the operator (3.6). Relations (3.7) and (3.8) imply that

$$
\left\|T_{\gamma}(t)\right\|_{\mathfrak{o}} \leq|t|(2 \delta)^{-1 / 2}\left\|X_{1}\right\|+t^{2}(2 \delta)^{-1}\left\|X_{1}\right\|^{2} .
$$

Combining this with (3.1), we obtain

$$
\left\|T_{\gamma}(t)\right\|_{\mathfrak{o}} \leq \frac{\sqrt{2}+1}{2}|t| \delta^{-1 / 2}\left\|X_{1}\right\|
$$

3.3. Estimate for the norm of the difference of resolvents. We start with the representation (2.11). By (2.14), (3.4), and (3.9), we obtain

$$
\left\|T_{\gamma}(t) R_{-\gamma}(t)\right\| \leq 4^{-1}(1+\sqrt{2}) \sqrt{2}|t| \delta^{-3 / 2}\left\|X_{1}\right\| .
$$

Combining this with (3.3) yields

$$
\left\|R_{z}(t)-R_{z}(0)\right\| \leq \beta_{1}^{\circ}|t| \delta^{-3 / 2}\left\|X_{1}\right\|, \quad|t| \leq t^{0}(\delta), \quad z \in \Gamma,
$$

where $\beta_{1}^{\circ}=5^{2} 2^{-1}\left(1+2^{-1 / 2}\right)$. In what follows, the bulky expressions for absolute constants will not be written down explicitly; these constants will be denoted by $\beta$ or $\beta^{\circ}$ with indices. However, it should be kept in mind that concrete numerical bounds can be given for these constants. 
3.4. The difference of resolvents. Separation of the main part. Estimate (3.10) does not suffice for our purposes. We separate the terms of the resolvent up to the order of $t^{2}$ and give an estimate of order $t^{3}$ for the remainder term. For this, we use a representation of the form (2.13):

$$
R_{z}(0)-R_{z}(t)=\Omega_{z}(0) T_{\gamma}(t) R_{z}(t), \quad z \in \Gamma .
$$

Here $|t| \leq t^{0}(\delta), \gamma=2 \delta$, and the operator $T_{\gamma}(t)$ is defined as in (3.6). The difference (3.11) can be written as follows:

$$
\begin{aligned}
R_{z}(0)-R_{z}(t) & =t \mathcal{I}_{1}+t^{2} \mathcal{I}_{2}, \\
\mathcal{I}_{k} & =\Omega_{z}(0) T_{\gamma}^{(k)} R_{z}(t), \quad k=1,2 .
\end{aligned}
$$

We start with the operator $\mathcal{I}_{2}$. Iterating $(3.11)$, we obtain

$$
\mathcal{I}_{2}=\Omega_{z}(0) T_{\gamma}^{(2)} R_{z}(0)-\Omega_{z}(0) T_{\gamma}^{(2)} \Omega_{z}(0) T_{\gamma}(t) R_{z}(t)=\mathcal{I}_{2}^{0}-\mathcal{I}_{2}^{(1)} .
$$

The operator

$$
\mathcal{I}_{2}^{0}=\Omega_{z}(0) T_{\gamma}^{(2)} R_{z}(0)
$$

does not depend on $t$, and for $\mathcal{I}_{2}^{(1)}$ it suffices to prove an estimate of order $|t|$. Such an estimate is a direct consequence of the corresponding inequality of the form (2.17). It suffices to use (3.8) and (3.9), and to refer to (3.3), (3.4), and the fact that $|z+\gamma| \gamma^{-1} \leq 2$ for $z \in \Gamma$. As a result, we obtain $\left\|\mathcal{I}_{2}^{(1)}\right\| \leq \beta_{2}^{\circ}|t| \delta^{-5 / 2}\left\|X_{1}\right\|^{3}$, whence

$$
t^{2} \mathcal{I}_{2}=t^{2} \mathcal{I}_{2}^{0}+\Psi_{2}^{0}(t), \quad\left\|\Psi_{2}^{0}(t)\right\| \leq \beta_{2}^{\circ}|t|^{3} \delta^{-5 / 2}\left\|X_{1}\right\|^{3} .
$$

3.5. Investigation of the operator $\mathcal{I}_{1}$. This is somewhat more cumbersome. We need to separate the zero and first order terms in $t$ from $\mathcal{I}_{1}$, and to efficiently control the remainder term. For this, we need to apply formula (3.11) twice. Namely, by (3.13), we have

$$
\mathcal{I}_{1}=\Omega_{z}(0) T_{\gamma}^{(1)} R_{z}(0)-\Omega_{z}(0) T_{\gamma}^{(1)} \Omega_{z}(0) T_{\gamma}(t) R_{z}(t)=\mathcal{I}_{1}^{0}-\mathcal{I}_{1}^{(1)} .
$$

Here

$$
\mathcal{I}_{1}^{0}=\Omega_{z}(0) T_{\gamma}^{(1)} R_{z}(0)
$$

does not depend on $t$. For the analysis of $\mathcal{I}_{1}^{(1)}$, we use (3.11) once again:

$$
\begin{aligned}
\mathcal{I}_{1}^{(1)}= & \Omega_{z}(0) T_{\gamma}^{(1)} \Omega_{z}(0) T_{\gamma}(t) R_{z}(0)-\Omega_{z}(0) T_{\gamma}^{(1)} \Omega_{z}(0) T_{\gamma}(t) \Omega_{z}(0) T_{\gamma}(t) R_{z}(t) \\
= & t \Omega_{z}(0) T_{\gamma}^{(1)} \Omega_{z}(0) T_{\gamma}^{(1)} R_{z}(0)+t^{2} \Omega_{z}(0) T_{\gamma}^{(1)} \Omega_{z}(0) T_{\gamma}^{(2)} R_{z}(0) \\
& -\Omega_{z}(0) T_{\gamma}^{(1)} T_{\gamma}(t) \Omega_{z}(0) T_{\gamma}(t) R_{z}(t) \\
& -(z+2 \delta) \Omega_{z}(0)\left(T_{\gamma}^{(1)} R_{z}(0)\right)\left(T_{\gamma}(t) \Omega_{z}(0) T_{\gamma}(t) R_{z}(t)\right) .
\end{aligned}
$$

The first term on the right is equal to $\mathcal{I}_{2}^{00}$, where

$$
\mathcal{I}_{2}^{00}=\Omega_{z}(0) T_{\gamma}^{(1)} \Omega_{z}(0) T_{\gamma}^{(1)} R_{z}(0),
$$

and the other three terms can be estimated with the help of (2.16), (2.17), and (3.7)-(3.9). All three terms give contributions of the same type. As a result, we obtain

$$
\begin{aligned}
t \mathcal{I}_{1} & =t \mathcal{I}_{1}^{0}-t^{2} \mathcal{I}_{2}^{00}+\Psi_{1}^{0}(t), \\
\left\|\Psi_{1}^{0}(t)\right\| & \leq \beta_{3}^{\circ}|t|^{3} \delta^{-5 / 2}\left\|X_{1}\right\|^{3} .
\end{aligned}
$$


3.6. The final result. From (3.15), (3.18), and (3.12) we obtain the following statement.

Theorem 3.1. Suppose condition (3.1) is fulfilled, $z \in \Gamma$, and $\gamma=2 \delta$. Then

$$
R_{z}(0)-R_{z}(t)=t \mathcal{I}_{1}^{0}+t^{2} \mathcal{I}_{2}^{+}+\Psi^{0}(t) .
$$

Here $\mathcal{I}_{2}^{+}=\mathcal{I}_{2}^{0}-\mathcal{I}_{2}^{00}$. The operators $\mathcal{I}_{1}^{0}, \mathcal{I}_{2}^{0}$, and $\mathcal{I}_{2}^{00}$ are defined by (3.16), (3.14), and (3.17), respectively. We have

$$
\left\|\Psi^{0}(t)\right\| \leq \beta^{\circ}|t|^{3} \delta^{-5 / 2}\left\|X_{1}\right\|^{3} .
$$

\section{$\S 4$. Threshold APproximations}

4.1. The difference of the spectral projections. We start with the representation

$$
F(t, \delta)=-\frac{1}{2 \pi i} \int_{\Gamma} R_{z}(t) d z .
$$

If $t=0$, the left-hand side of (4.1) is equal to $P$; therefore,

$$
F(t, \delta)-P=-\frac{1}{2 \pi i} \int_{\Gamma}\left(R_{z}(t)-R_{z}(0)\right) d z .
$$

Directly from (3.10) and (4.2), it follows that

$$
F(t, \delta)-P=\Phi(t), \quad\|\Phi(t)\| \leq \beta_{1}|t| \delta^{-1 / 2}\left\|X_{1}\right\|, \quad|t| \leq t^{0}(\delta) .
$$

Thus, we have proved the following theorem.

Theorem 4.1. Let $X(t)$ and $A(t)$ be the operator families introduced in Subsection 1.1. Suppose that $8 \delta<d^{0}$, where $d^{0}$ is the distance between the point $\lambda=0$ and the remaining part of the spectrum of $A_{0}=A(0)$. Finally, suppose that condition (3.1) is satisfied. If $F(t, \delta)$ is the spectral projection for $A(t)$ corresponding to the interval $[0, \delta]$, and $P$ is the orthogonal projection onto $\operatorname{Ker} A_{0}$, then estimate (4.3) is true.

Remark 4.2. 1) Along with (4.3), we have the trivial estimate $\|\Phi(t)\| \leq 2$.

2) The right-hand side of (4.3) can be represented as $\beta_{1}|t| / t^{0}(\delta)$.

4.2. Approximation for $A(t) F(t, \delta)$. For $t=0$, the left-hand side in the representation

$$
A(t) F(t, \delta)=-\frac{1}{2 \pi i} \int_{\Gamma} z R_{z}(t) d z
$$

is equal to $A_{0} P=0$; therefore,

$$
A(t) F(t, \delta)=\frac{1}{2 \pi i} \int_{\Gamma} z\left(R_{z}(0)-R_{z}(t)\right) d z .
$$

By (3.19), from (4.4) we deduce that

$$
A(t) F(t, \delta)=t I_{1}+t^{2} I_{2}+\Psi(t), \quad|t| \leq t^{0}(\delta),
$$

where $I_{1}$ and $I_{2}$ are bounded operators independent of $t$, and

$$
\|\Psi(t)\| \leq \beta_{2}|t|^{3} \delta^{-1 / 2}\left\|X_{1}\right\|^{3}, \quad|t| \leq t^{0}(\delta) .
$$

The coefficients $I_{1}$ and $I_{2}$ can be expressed via integrals of $z \mathcal{I}_{1}^{0}, z \mathcal{I}_{2}^{+}$over $\Gamma$. However, it is easier to find these coefficients by comparing (4.5) and (1.38). This yields $I_{1}=0$, $I_{2}=S P$, and the following theorem is true.

Theorem 4.3. Under the assumptions of Theorem 4.1, we have

$$
A(t) F(t, \delta)-t^{2} S P=\Psi(t), \quad|t| \leq t^{0}(\delta),
$$

where $\Psi(t)$ satisfies estimate (4.6). 
Remark 4.4. In Theorem 4.3, the germ $S$ is not assumed to be nondegenerate.

Now, let $\widetilde{A}(t)$ be yet another operator family in $\mathfrak{H}$ of the same type as $A(t)$. All the objects related to the family $\widetilde{A}$ will be marked by " $\sim$ ". Relations (4.6) and (4.7) imply the following theorem.

Theorem 4.5. Suppose that $8 \delta<\min \left(d^{0}, \widetilde{d}^{0}\right)$, and that the families $A$ and $\widetilde{A}$ are threshold equivalent, i.e., $\mathfrak{N}=\widetilde{\mathfrak{N}}$ and $S=\widetilde{S}$. Then

$$
\begin{array}{r}
\|A(t) F(t, \delta)-\widetilde{A}(t) \widetilde{F}(t, \delta)\| \leq \beta_{2}|t|^{3} \delta^{-1 / 2}\left(\left\|X_{1}\right\|^{3}+\left\|\widetilde{X}_{1}\right\|^{3}\right), \\
|t| \leq \delta^{1 / 2} \min \left(\left\|X_{1}\right\|^{-1},\left\|\widetilde{X}_{1}\right\|^{-1}\right) .
\end{array}
$$

4.3. Approximation for the imaginary exponential. For $\tau \in \mathbb{R}$, consider the group $\exp (-i \tau A(t))$. We put

$$
\begin{aligned}
& E(\tau):=(\exp (-i \tau A(t))) F(t, \delta)-\left(\exp \left(-i \tau t^{2} S P\right)\right) P, \\
& \Sigma(\tau):=\left(\exp \left(i t^{2} \tau S P\right)\right) E(\tau)=\left(\exp \left(i t^{2} \tau S P\right)\right) F(t, \delta)(\exp (-i \tau A(t)))-P .
\end{aligned}
$$

Note that $\Sigma(0)=F(t, \delta)-P=\Phi(t)$. Next, we have

$$
\frac{d \Sigma(\tau)}{d \tau}=i e^{i t^{2} \tau S P}\left(t^{2} S P F(t, \delta)-F(t, \delta) A(t)\right) e^{-i \tau A(t)},
$$

whence $\left\|\Sigma^{\prime}(\tau)\right\|=\|\Psi(t) F(t, \delta)\| \leq\|\Psi(t)\|$ and $\|E(\tau)\|=\|\Sigma(\tau)\| \leq\|\Phi(t)\|+|\tau|\|\Psi(t)\|$, where $\Phi(t)$ and $\Psi(t)$ satisfy estimates (4.3) and (4.6). This proves the following statement.

Theorem 4.6. Under the assumptions of Theorem 4.1, the operator-valued function (4.8) satisfies the estimate

$$
\|E(\tau)\| \leq \beta_{1}|t| \delta^{-1 / 2}\left\|X_{1}\right\|+\beta_{2}|\tau||t|^{3} \delta^{-1 / 2}\left\|X_{1}\right\|^{3}, \quad|t| \leq t^{0}(\delta) .
$$

Theorem 4.6 automatically implies a statement similar to Theorem 4.5 , but we do not dwell on this.

\section{$\S 5$. Approximation for the operator-Valued function $\left(A(t)+\varepsilon^{2} I\right)^{-1}$}

5.1. Statement of the problem. Our goal is to approximate the resolvent of $A(t)$ by the resolvent of the corresponding germ $S$. As compared to the assumptions of Theorems 4.1 and 4.3, we now need an additional condition. Namely, we assume that

$$
A(t) F(t, \delta) \geq c_{*} t^{2} F(t, \delta), \quad c_{*}>0, \quad|t| \leq t^{0}(\delta) .
$$

Condition (5.1) is equivalent to the following estimate for the eigenvalues $\lambda_{l}(t), l=$ $1, \ldots, n$, of the family $A(t)$ introduced in (1.27):

$$
\lambda_{l}(t) \geq c_{*} t^{2}, \quad l=1, \ldots, n, \quad c_{*}>0, \quad|t| \leq t^{0}(\delta) .
$$

Clearly, under this condition, the germ $S$ of the family $A(t)$ is nondegenerate, and inequalities (1.36) are valid with the same constant $c_{*}$ as in (5.2). In other words, $S \geq c_{*} I_{\mathfrak{N}}$, where $I_{\mathfrak{N}}=\left.P\right|_{\mathfrak{N}}$ is the identity operator in $\mathfrak{N}$.

Remark 5.1. In fact, by (1.28), inequalities (1.36) yield inequalities of the form (5.2), but with a smaller constant $c_{*}$ and for $t$ belonging to a smaller interval. In applications, it is often more convenient to check (5.2) directly.

We shall estimate the norm of the operator-valued function

$$
G(\varepsilon, t, \delta):=\left(A(t)+\varepsilon^{2} I\right)^{-1} F(t, \delta)-\left(t^{2} S P+\varepsilon^{2} I\right)^{-1} P, \quad \varepsilon>0,|t| \leq t^{0}(\delta) .
$$

First, we note that

$$
\left(t^{2} S P+\varepsilon^{2} I\right)^{-1} P=\left(t^{2} S+\varepsilon^{2} I_{\mathfrak{N}}\right)^{-1} P,
$$

where $t^{2} S+\varepsilon^{2} I_{\mathfrak{N}}$ is viewed as an operator in $\mathfrak{N}$. 
The following estimates are direct consequences of (5.1) and (1.36):

$$
\begin{array}{ll}
\left\|\left(A(t)+\varepsilon^{2} I\right)^{-1}\right\| \leq\left(c_{*} t^{2}+\varepsilon^{2}\right)^{-1}, & |t| \leq t^{0}(\delta), \\
\left\|\left(t^{2} S+\varepsilon^{2} I_{\mathfrak{N}}\right)^{-1}\right\| \leq\left(c_{*} t^{2}+\varepsilon^{2}\right)^{-1}, & |t| \leq t^{0}(\delta) .
\end{array}
$$

For a while, we shall use the abridged notation

$$
A(t)=A, \quad F(t, \delta)=F, \quad G(\varepsilon, t, \delta)=G, \quad|t| \leq t^{0}(\delta) .
$$

It is convenient to separate the factor depending on $|t|$ in estimates (4.3) and (4.6). We have

$$
\begin{aligned}
& \|\Phi(t)\| \leq \mathcal{C}_{1}|t|, \quad \mathcal{C}_{1}=\beta_{1} \delta^{-1 / 2}\left\|X_{1}\right\|=\beta_{1}\left(t^{0}(\delta)\right)^{-1}, \quad|t| \leq t^{0}(\delta), \\
& \|\Psi(t)\| \leq \mathcal{C}_{2}|t|^{3}, \quad \mathcal{C}_{2}=\beta_{2} \delta^{-1 / 2}\left\|X_{1}\right\|^{3}=\beta_{2} \delta\left(t^{0}(\delta)\right)^{-3}, \quad|t| \leq t^{0}(\delta) .
\end{aligned}
$$

Finally, we write the obvious identity

$$
\left(A+\varepsilon^{2} I\right)^{-1} F=\left(A F+\varepsilon^{2} I\right)^{-1} F .
$$

5.2. Approximation of the resolvent near the threshold. Our nearest goal is the proof of the following theorem.

Theorem 5.2. Under condition (5.2), the operator-valued function (5.3) satisfies the estimate

$$
\|G(\varepsilon, t, \delta)\| \leq \mathcal{C}_{0}|t|\left(c_{*} t^{2}+\varepsilon^{2}\right)^{-1}, \quad \varepsilon>0, \quad|t| \leq t^{0}(\delta), \quad \mathcal{C}_{0}=2 \mathcal{C}_{1}+c_{*}^{-1} \mathcal{C}_{2},
$$

and, consequently,

$$
2 \varepsilon\|G(\varepsilon, t, \delta)\| \leq \mathcal{C}, \quad \mathcal{C}=c_{*}^{-1 / 2} \mathcal{C}_{0}, \quad \varepsilon>0, \quad|t| \leq t^{0}(\delta) .
$$

Here $c_{*}$ is the same constant as in (5.2), and $\mathcal{C}_{1}, \mathcal{C}_{2}$ are defined by (5.6), (5.7).

Proof. We start with the identity

$$
\begin{gathered}
F\left(A F+\varepsilon^{2} I\right)^{-1}\left(A F+\varepsilon^{2} I-\left(t^{2} S P+\varepsilon^{2} I\right)\right)\left(t^{2} S P+\varepsilon^{2} I\right)^{-1} P \\
=F\left(t^{2} S P+\varepsilon^{2} I\right)^{-1} P-F\left(A F+\varepsilon^{2} I\right)^{-1} P .
\end{gathered}
$$

By (5.8), this implies that $G=G_{1}+G_{2}+G_{0}$, where

$$
\begin{aligned}
& G_{1}=\left(A+\varepsilon^{2} I\right)^{-1} F \Phi(t), \\
& G_{2}=\Phi(t)\left(t^{2} S P+\varepsilon^{2} I\right)^{-1} P, \\
& G_{0}=-F\left(A+\varepsilon^{2} I\right)^{-1} \Psi(t)\left(t^{2} S P+\varepsilon^{2} I\right)^{-1} P .
\end{aligned}
$$

Recall that the operators $\Phi(t)=F-P$ and $\Psi(t)=A F-t^{2} S P$ satisfy (5.6) and (5.7). From (5.4)-(5.7) we see that, for $\varepsilon>0$ and $|t| \leq t^{0}(\delta)$,

$$
\begin{aligned}
\left\|G_{1}\right\|+\left\|G_{2}\right\| & \leq 2 \mathcal{C}_{1}\left(c_{*} t^{2}+\varepsilon^{2}\right)^{-1}|t|, \\
\left\|G_{0}\right\| & \leq \mathcal{C}_{2}\left(c_{*} t^{2}+\varepsilon^{2}\right)^{-2}|t|^{3} \\
& \leq c_{*}^{-1} \mathcal{C}_{2}\left(c_{*} t^{2}+\varepsilon^{2}\right)^{-1}|t|,
\end{aligned}
$$

and estimate (5.9) follows.

Remark 5.3. Estimate (5.9) is two-parametric. For $t$ fixed, the operator-valued function $G(\varepsilon, t, \delta)$ is bounded as $\varepsilon \rightarrow 0$. Estimate (5.10) (which follows from (5.9)) is uniform in $t$ for $|t| \leq t^{0}(\delta)$.

Now, let $\widetilde{A}(t)$ be a new family of the form (1.2) satisfying the analog of condition (5.1). Estimate (5.10) implies the following statement. 
Theorem 5.4. Suppose the families $A(t)$ and $\widetilde{A}(t)$ are threshold equivalent and $8 \delta<$ $\min \left\{d^{0}, \widetilde{d}^{0}\right\}$. Then, for $\varepsilon>0$ and $|t| \leq \delta^{1 / 2} \min \left\{\left\|X_{1}\right\|^{-1},\left\|\widetilde{X}_{1}\right\|^{-1}\right\}=\min \left\{t^{0}(\delta), \widetilde{t}^{0}(\delta)\right\}$, the operator-valued function

$$
J=\left(A(t)+\varepsilon^{2} I\right)^{-1} F(t, \delta)-\left(\widetilde{A}(t)+\varepsilon^{2} I\right)^{-1} \widetilde{F}(t, \delta)
$$

satisfies the estimate

$$
2 \varepsilon\|J\| \leq \mathcal{C}+\widetilde{\mathcal{C}}=c_{*}^{-1 / 2}\left(2 \mathcal{C}_{1}+c_{*}^{-1} \mathcal{C}_{2}\right)+\widetilde{c}_{*}^{-1 / 2}\left(2 \widetilde{\mathcal{C}}_{1}+\widetilde{c}_{*}^{-1} \widetilde{\mathcal{C}}_{2}\right) .
$$

By Proposition 1.2, we have $\left\|\left(A(t)+\varepsilon^{2} I\right)^{-1} F(t, \delta)^{\perp}\right\| \leq(3 \delta)^{-1}$. Therefore, the following two statements are direct consequences of Theorems 5.2 and 5.4.

Theorem 5.5. Under the assumptions of Theorem 5.2, we have

$$
\varepsilon\left\|\left(A(t)+\varepsilon^{2} I\right)^{-1}-\left(t^{2} S P+\varepsilon^{2} I\right)^{-1} P\right\| \leq 2^{-1} \mathcal{C}+\varepsilon(3 \delta)^{-1}, \quad \varepsilon>0, \quad|t| \leq t^{0}(\delta) .
$$

Theorem 5.6. Under the assumptions of Theorem 5.4, we have

$$
\varepsilon\left\|\left(A(t)+\varepsilon^{2} I\right)^{-1}-\left(\widetilde{A}(t)+\varepsilon^{2} I\right)^{-1}\right\| \leq 2^{-1}(\mathcal{C}+\widetilde{\mathcal{C}})+2 \varepsilon(3 \delta)^{-1}, \quad \varepsilon>0, \quad|t| \leq t^{0}(\delta) .
$$

5.3. Approximation of the generalized resolvent near the threshold. 1 Theorem 5.5 can be extended to the case where the resolvent $\left(A(t)+\varepsilon^{2} I\right)^{-1}$ is replaced by the operator $\left(\widehat{A}(t)+\varepsilon^{2} Q\right)^{-1}$. Here $Q$ is a bounded positive definite operator in $\widehat{\mathfrak{H}}$. This useful generalization will be deduced from Theorem 5.5 on the basis of Subsection 1.5. We shall use the notation and material of Subsection 1.5 without repeating the explanations. We only note that, by (1.20),

$$
M\left(A(t)+\varepsilon^{2} I\right)^{-1} M^{*}=\left(\widehat{A}(t)+\varepsilon^{2} Q\right)^{-1},
$$

where

$$
Q=\left(M^{*}\right)^{-1} M^{-1}=\left(M^{*}\right)^{-1}: \widehat{\mathfrak{H}} \rightarrow \widehat{\mathfrak{H}}
$$

We start with preliminary remarks. Since $\widehat{P}^{\perp} M P=0$, we have $P M^{*} \widehat{P}^{\perp}=0$. Consequently,

$$
P M^{*} \widehat{P}=P M^{*} .
$$

Next, since $M P M^{-1} \widehat{P}=\widehat{P}$, we have

$$
\widehat{P}=\widehat{P}\left(M^{-1}\right)^{*} P M^{*} .
$$

Consider the block $Q_{\widehat{\mathfrak{N}}}$ of the operator $Q$ in the subspace $\widehat{\mathfrak{N}}$ :

$$
Q_{\widehat{\mathfrak{N}}}=\left.\widehat{P} Q\right|_{\widehat{\mathfrak{N}}}: \widehat{\mathfrak{N}} \rightarrow \widehat{\mathfrak{N}} .
$$

By (5.2), the germ $S$ of the family $A(t)$ is nondegenerate. Then so is the germ $\widehat{S}$ of $\widehat{A}(t)$. Therefore, the operator

$$
\left(t^{2} \widehat{S}+\varepsilon^{2} Q_{\widehat{\mathfrak{N}}}\right)^{-1}: \widehat{\mathfrak{N}} \rightarrow \widehat{\mathfrak{N}}
$$

exists.

Proposition 5.7. We have

$$
M\left(t^{2} S P+\varepsilon^{2} I\right)^{-1} P M^{*}=\left(t^{2} \widehat{S}+\varepsilon^{2} Q_{\widehat{\mathfrak{N}}}\right)^{-1} \widehat{P} .
$$

\footnotetext{
${ }^{1}$ The results of this subsection were obtained by the authors with the participation of R. G. Shterenberg.
} 
Proof. Both operators in (5.18) act in $\widehat{\mathfrak{H}}$ and take $\widehat{\mathfrak{N}}$ into itself. The operator on the left can be represented (cf. (5.15)) as

$$
\widehat{P} M\left(t^{2} S P+\varepsilon^{2} I\right)^{-1} P M^{*} \widehat{P} .
$$

We put $\widehat{\zeta}=M\left(t^{2} S P+\varepsilon^{2} I\right)^{-1} P M^{*} \widehat{\eta}$. Suppose $\widehat{\eta} \in \widehat{\mathfrak{N}}$; then $\widehat{\zeta} \in \widehat{\mathfrak{N}}$ and $P M^{*} \widehat{\eta}=$ $\left(t^{2} S P+\varepsilon^{2} I\right) M^{-1} \widehat{\zeta}$. By (5.14), (5.16), (5.17), and also (1.24), we have

$$
\widehat{\eta}=t^{2} \widehat{P}\left(M^{*}\right)^{-1} S M^{-1} \widehat{\zeta}+\varepsilon^{2} \widehat{P}\left(M^{*}\right)^{-1} M^{-1} \widehat{\zeta}=\left(t^{2} \widehat{S}+\varepsilon^{2} Q_{\widehat{\mathfrak{N}}}\right) \widehat{\zeta}
$$

Thus, $\widehat{\zeta}=\left(t^{2} \widehat{S}+\varepsilon^{2} Q_{\widehat{\mathfrak{N}}}\right)^{-1} \widehat{\eta}$, which is equivalent to (5.18).

For the operator-valued function

$$
\mathcal{Y}(\varepsilon, t)=\left(A(t)+\varepsilon^{2} I\right)^{-1}-\left(t^{2} S P+\varepsilon^{2} I\right)^{-1} P,
$$

we write (5.11) as

$$
\varepsilon\|\mathcal{Y}(\varepsilon, t)\| \leq C, \quad C=2^{-1} \mathcal{C}+(3 \delta)^{-1}, \quad 0<\varepsilon \leq 1, \quad|t| \leq t^{0}(\delta) .
$$

By (5.13), (5.18), and (5.19),

$$
M \mathcal{Y}(\varepsilon, t) M^{*}=\left(\widehat{A}(t)+\varepsilon^{2} Q\right)^{-1}-\left(t^{2} \widehat{S}+\varepsilon^{2} Q_{\widehat{\mathfrak{N}}}\right)^{-1} \widehat{P} .
$$

Combining this with (5.20), we obtain

$$
\varepsilon\left\|\left(\widehat{A}(t)+\varepsilon^{2} Q\right)^{-1}-\left(t^{2} \widehat{S}+\varepsilon^{2} Q_{\widehat{\mathfrak{N}}}\right)^{-1} \widehat{P}\right\| \leq C\|M\|^{2}=C\left\|Q^{-1}\right\| .
$$

All quantities in (5.21) are expressed in terms of the family $\widehat{A}(t)$ and the operator $Q$, which act in $\widehat{\mathfrak{H}}$. Thus, estimate $(5.21)$ can be treated as a generalization of (5.20) for the operator-valued function (5.19). However, the constant $C$ comes from the initial family. This constant can be recalculated (with making it rougher), but, for applications, we do not need this.

Now, let $\widehat{A}_{+}(t)$ be yet another family of the form $\widehat{A}(t)$ acting in $\widehat{\mathfrak{H}}$, and let $Q_{+}$be a bounded positive definite operator in $\widehat{\mathfrak{H}}$. Suppose that the families $\widehat{A}(t)$ and $\widehat{A}_{+}(t)$ are threshold equivalent and that the blocks of the operators $Q$ and $Q_{+}$in $\widehat{\mathfrak{N}}$ coincide. Next, let $M_{+}=\left(Q_{+}\right)^{-1 / 2}$ and $A_{+}(t)=M_{+} \widehat{A}_{+}(t) M_{+}$, and let $C_{+}$be the analog of the constant $C$ (see (5.20)) for the family $A_{+}(t)$.

Theorem 5.8. Under the above conditions, we have

$$
\varepsilon\left\|\left(\widehat{A}(t)+\varepsilon^{2} Q\right)^{-1}-\left(\widehat{A}_{+}(t)+\varepsilon^{2} Q_{+}\right)^{-1}\right\| \leq C\left\|Q^{-1}\right\|+C_{+}\left\|Q_{+}^{-1}\right\|,
$$

where $0<\varepsilon \leq 1,8 \delta<\min \left\{d^{0}, d_{+}^{0}\right\}$, and $|t| \leq \min \left\{t^{0}(\delta), t_{+}^{0}(\delta)\right\}$.

Finally, (5.13), (5.14), and (5.22) imply the following statement.

Theorem 5.9. Under the assumptions of Theorem 5.8, we have

$$
\varepsilon\left\|\left(A(t)+\varepsilon^{2} I\right)^{-1}-M^{-1}\left(\widehat{A}_{+}(t)+\varepsilon^{2} Q_{+}\right)^{-1}\left(M^{*}\right)^{-1}\right\| \leq\left\|M^{-1}\right\|^{2}\left(C\|M\|^{2}+C_{+}\left\|Q_{+}^{-1}\right\|\right) .
$$

Chapter 2. Periodic differential operators in $L_{2}\left(\mathbb{R}^{d} ; \mathbb{C}^{n}\right)$

\section{$\S 1$. Main definitions. Preliminaries}

1.1. Factorized second order operators. We consider selfadjoint matrix second order DO's represented as a product of two mutually adjoint first order DO's. Let $b(\mathbf{D})$ : $L_{2}\left(\mathbb{R}^{d} ; \mathbb{C}^{n}\right) \rightarrow L_{2}\left(\mathbb{R}^{d} ; \mathbb{C}^{m}\right)$ be a homogeneous first order DO with constant coefficients. We always assume that

$$
m \geq n
$$


We fix orthonormal bases $\widetilde{\mathbf{e}}_{1}, \ldots, \widetilde{\mathbf{e}}_{n}$ in $\mathbb{C}^{n}, \mathbf{e}_{1}, \ldots, \mathbf{e}_{m}$ in $\mathbb{C}^{m}$, and $\mathbf{e}_{1}^{\prime}, \ldots, \mathbf{e}_{d}^{\prime}$ in $\mathbb{R}^{d}$. Then the symbol $b(\boldsymbol{\xi})$ is associated with the operator $b(\mathbf{D})$. This symbol is an $(m \times n)$-matrixvalued linear homogeneous function of $\xi \in \mathbb{R}^{d}$. Suppose that

$$
\operatorname{rank} b(\xi)=n, \quad 0 \neq \xi \in \mathbb{R}^{d} ;
$$

then

$$
\alpha_{0} \mathbf{1}_{n} \leq b(\boldsymbol{\theta})^{*} b(\boldsymbol{\theta}) \leq \alpha_{1} \mathbf{1}_{n}, \quad|\boldsymbol{\theta}|=1, \quad 0<\alpha_{0} \leq \alpha_{1}<\infty
$$

Note that

$$
b(\boldsymbol{\xi})=\sum_{j=1}^{d} \xi^{j} b_{j}, \quad \xi=\sum_{j=1}^{d} \xi^{j} \mathbf{e}_{j}^{\prime},
$$

where the $b_{j}$ are constant $(m \times n)$-matrices with $\operatorname{rank} b_{j}=n$.

Suppose that an $(n \times n)$-matrix-valued function $f(\mathbf{x})$ and an $(m \times m)$-matrix-valued function $h(\mathbf{x}), \mathbf{x} \in \mathbb{R}^{d}$, are bounded, together with their inverses:

$$
f, f^{-1} \in L_{\infty}\left(\mathbb{R}^{d}\right) ; \quad h, h^{-1} \in L_{\infty}\left(\mathbb{R}^{d}\right) .
$$

We consider the DO

$$
\begin{gathered}
\mathcal{X}:=h b(\mathbf{D}) f: L_{2}\left(\mathbb{R}^{d} ; \mathbb{C}^{n}\right) \rightarrow L_{2}\left(\mathbb{R}^{d} ; \mathbb{C}^{m}\right), \\
\operatorname{Dom} \mathcal{X}:=\left\{\mathbf{u} \in L_{2}\left(\mathbb{R}^{d} ; \mathbb{C}^{n}\right): f \mathbf{u} \in H^{1}\left(\mathbb{R}^{d} ; \mathbb{C}^{n}\right)\right\} .
\end{gathered}
$$

The operator (1.6) is closed on the domain (1.7). The selfadjoint operator

$$
\mathcal{A}:=\mathcal{X}^{*} \mathcal{X}
$$

in $L_{2}\left(\mathbb{R}^{d} ; \mathbb{C}^{n}\right)$ is generated by the closed quadratic form

$$
a[\mathbf{u}, \mathbf{u}]:=\|\mathcal{X} \mathbf{u}\|_{L_{2}\left(\mathbb{R}^{d} ; \mathbb{C}^{m}\right)}^{2}, \quad \mathbf{u} \in \operatorname{Dom} \mathcal{X} .
$$

Formally,

$$
\mathcal{A}=f(\mathbf{x})^{*} b(\mathbf{D})^{*} g(\mathbf{x}) b(\mathbf{D}) f(\mathbf{x}), \quad g(\mathbf{x}):=h(\mathbf{x})^{*} h(\mathbf{x}) .
$$

By using the Fourier transformation and conditions (1.3) and (1.5), it is easy to show that

$$
\begin{gathered}
c_{0} \int_{\mathbb{R}^{d}}|\mathbf{D}(f \mathbf{u})|^{2} d \mathbf{x} \leq a[\mathbf{u}, \mathbf{u}] \leq c_{1} \int_{\mathbb{R}^{d}}|\mathbf{D}(f \mathbf{u})|^{2} d \mathbf{x}, \quad \mathbf{u} \in \operatorname{Dom} \mathcal{X}, \\
c_{0}=\alpha_{0}\left\|h^{-1}\right\|_{L_{\infty}}^{-2}, \quad c_{1}=\alpha_{1}\|h\|_{L_{\infty}}^{2} .
\end{gathered}
$$

1.2. The lattices $\Gamma$ and $\widetilde{\Gamma}$. In what follows, the functions $f$ and $h$ (and, with them, the operators $\mathcal{X}, \mathcal{A}$ ) are assumed to be periodic with respect to some lattice $\Gamma \subset \mathbb{R}^{d}$ :

$$
f(\mathbf{x}+\mathbf{a})=f(\mathbf{x}), \quad h(\mathbf{x}+\mathbf{a})=h(\mathbf{x}), \quad \mathbf{a} \in \Gamma .
$$

Let $\mathbf{a}_{1}, \ldots, \mathbf{a}_{d} \in \mathbb{R}^{d}$ be the basis in $\mathbb{R}^{d}$ that generates the lattice $\Gamma$, i.e.,

$$
\Gamma=\left\{\mathbf{a} \in \mathbb{R}^{d}: \mathbf{a}=\sum_{j=1}^{d} \nu^{j} \mathbf{a}_{j}, \nu^{j} \in \mathbb{Z}\right\},
$$

and let $\Omega$ be the (elementary) cell of $\Gamma$ :

$$
\Omega:=\left\{\mathbf{x} \in \mathbb{R}^{d}: \mathbf{x}=\sum_{j=1}^{d} \tau^{j} \mathbf{a}_{j}, 0<\tau^{j}<1\right\} .
$$


The basis $\mathbf{b}^{1}, \ldots, \mathbf{b}^{d}$ in $\mathbb{R}^{d}$ dual to $\mathbf{a}_{1}, \ldots, \mathbf{a}_{d}$ is defined by the relations $\left\langle\mathbf{b}^{i}, \mathbf{a}_{j}\right\rangle=2 \pi \delta_{j}^{i}$. This basis generates the lattice $\widetilde{\Gamma}$ dual to $\Gamma$ :

$$
\widetilde{\Gamma}=\left\{\mathbf{b} \in \mathbb{R}^{d}: \mathbf{b}=\sum_{i=1}^{d} \mu_{i} \mathbf{b}^{i}, \mu_{i} \in \mathbb{Z}\right\} .
$$

The cell of $\widetilde{\Gamma}$ can be defined by analogy with (1.13). However, it is more convenient to denote by $\widetilde{\Omega}$ the Brillouin zone

$$
\widetilde{\Omega}=\left\{\mathbf{k} \in \mathbb{R}^{d}:|\mathbf{k}|<|\mathbf{k}-\mathbf{b}|, 0 \neq \mathbf{b} \in \widetilde{\Gamma}\right\} .
$$

Like the cell, the domain $\widetilde{\Omega}$ is a fundamental domain for $\widetilde{\Gamma}$. This means that all $\widetilde{\Gamma}$-shifted copies of $\widetilde{\Omega}$ are mutually disjoint and the union of the $\widetilde{\Gamma}$-shifted copies of clos $\widetilde{\Omega}$ covers $\mathbb{R}^{d}$. The closure clos $\widetilde{\Omega}$ is a convex polyhedron. The points $\mathbf{k} \in \partial \widetilde{\Omega}$ are characterized by the relation $|\mathbf{k}|=\min |\mathbf{k}-\mathbf{b}|, 0 \neq \mathbf{b} \in \widetilde{\Gamma}$. We shall use the notation $|\Omega|=$ meas $\Omega$, $|\widetilde{\Omega}|=$ meas $\widetilde{\Omega}$. Note that $|\Omega||\widetilde{\Omega}|=(2 \pi)^{d}$. Let $r_{0}$ be the radius of the ball inscribed in $\operatorname{clos} \widetilde{\Omega}$. Then

$$
2 r_{0}=\min |\mathbf{b}|, \quad 0 \neq \mathbf{b} \in \widetilde{\Gamma}
$$

We denote

$$
\mathcal{K}(r)=\left\{\mathbf{k} \in \mathbb{R}^{d}:|\mathbf{k}| \leq r\right\}, \quad 0<r \leq r_{0} .
$$

Associated with the lattice $\Gamma$ is the discrete Fourier transformation

$$
v(\mathbf{x})=|\Omega|^{-1 / 2} \sum_{\mathbf{b} \in \widetilde{\Gamma}} v_{\mathbf{b}} \exp (i\langle\mathbf{b}, \mathbf{x}\rangle), \quad \mathbf{x} \in \Omega .
$$

This transformation maps $l_{2}(\widetilde{\Gamma})$ onto $L_{2}(\Omega)$ unitarily:

$$
\int_{\Omega}|v(\mathbf{x})|^{2} d \mathbf{x}=\sum_{\mathbf{b} \in \widetilde{\Gamma}}\left|v_{\mathbf{b}}\right|^{2} .
$$

Below, $\widetilde{H}^{1}(\Omega)$ stands for the subspace of all functions in $H^{1}(\Omega)$ such that the $\Gamma$-periodic extension of them to $\mathbb{R}^{d}$ belongs to $H_{\mathrm{loc}}^{1}\left(\mathbb{R}^{d}\right)$. We have

$$
\int_{\Omega}|((\mathbf{D}+\mathbf{k}) \mathbf{v})(\mathbf{x})|^{2} d \mathbf{x}=\sum_{\mathbf{b} \in \widetilde{\Gamma}}|\mathbf{b}+\mathbf{k}|^{2}\left|\mathbf{v}_{\mathbf{b}}\right|^{2}, \quad \mathbf{v} \in \widetilde{H}^{1}\left(\Omega ; \mathbb{C}^{n}\right), \mathbf{k} \in \mathbb{R}^{d} .
$$

The convergence of the series in (1.16) is equivalent to the fact that $\mathbf{v} \in \widetilde{H}^{1}\left(\Omega ; \mathbb{C}^{n}\right)$. We mention a more general relation. Let $b(\xi)$ be the symbol introduced in Subsection 1.1. Then

$$
\int_{\Omega}|b(\mathbf{D}+\mathbf{k}) \mathbf{v}|^{2} d \mathbf{x}=\sum_{\mathbf{b} \in \widetilde{\Gamma}}\left|b(\mathbf{b}+\mathbf{k}) \mathbf{v}_{\mathbf{b}}\right|^{2}, \quad \mathbf{v} \in \widetilde{H}^{1}\left(\Omega ; \mathbb{C}^{n}\right), \mathbf{k} \in \mathbb{R}^{d} .
$$

1.3. The Gelfand transformation. Initially, the Gelfand transformation $\mathcal{U}$ is defined on the functions of the Schwartz class $\mathcal{S}$ by the formula

$$
\begin{array}{r}
\widetilde{\mathbf{v}}(\mathbf{k}, \mathbf{x})=(\mathcal{U} \mathbf{v})(\mathbf{k}, \mathbf{x})=|\widetilde{\Omega}|^{-1 / 2} \sum_{\mathbf{a} \in \Gamma} \exp (-i\langle\mathbf{k}, \mathbf{x}+\mathbf{a}\rangle) \mathbf{v}(\mathbf{x}+\mathbf{a}), \\
\mathbf{v} \in \mathcal{S}\left(\mathbb{R}^{d} ; \mathbb{C}^{n}\right), \mathbf{x} \in \mathbb{R}^{d}, \mathbf{k} \in \mathbb{R}^{d} .
\end{array}
$$

Since

$$
\int_{\widetilde{\Omega}} \int_{\Omega}|\widetilde{\mathbf{v}}(\mathbf{k}, \mathbf{x})|^{2} d \mathbf{x} d \mathbf{k}=\int_{\mathbb{R}^{d}}|\mathbf{v}(\mathbf{x})|^{2} d \mathbf{x}, \quad \widetilde{\mathbf{v}}=\mathcal{U} \mathbf{v}
$$


$\mathcal{U}$ extends by continuity to a unitary mapping

$$
\mathcal{U}: L_{2}\left(\mathbb{R}^{d} ; \mathbb{C}^{n}\right) \rightarrow \int_{\widetilde{\Omega}} \oplus L_{2}\left(\Omega ; \mathbb{C}^{n}\right) d \mathbf{k}=: \mathcal{H}
$$

Next, the relation $\mathbf{v} \in H^{1}\left(\mathbb{R}^{d} ; \mathbb{C}^{n}\right)$ is equivalent to the fact that $\widetilde{\mathbf{v}}(\mathbf{k}, \cdot) \in \widetilde{H}^{1}(\Omega)$ for almost every $\mathbf{k} \in \widetilde{\Omega}$ and

$$
\int_{\widetilde{\Omega}} \int_{\Omega}\left(|(\mathbf{D}+\mathbf{k}) \widetilde{\mathbf{v}}(\mathbf{k}, \mathbf{x})|^{2}+|\widetilde{\mathbf{v}}(\mathbf{k}, \mathbf{x})|^{2}\right) d \mathbf{x} d \mathbf{k}<\infty
$$

Under the transformation $\mathcal{U}$, the operator of multiplication by a bounded periodic function in $L_{2}\left(\mathbb{R}^{d} ; \mathbb{C}^{n}\right)$ turns into multiplication by the same function on the fibers of the direct integral $\mathcal{H}$ (see (1.18)). On these fibers, the operator $b(\mathbf{D})$ applied to $\mathbf{v} \in H^{1}\left(\mathbb{R}^{d} ; \mathbb{C}^{n}\right)$ turns into the operator $b(\mathbf{D}+\mathbf{k})$ applied to $\widetilde{\mathbf{v}}(\mathbf{k}, \cdot) \in \widetilde{H}^{1}\left(\Omega ; \mathbb{C}^{n}\right)$.

\section{§2. Direct integral expansion for the operator $\mathcal{A}$}

2.1. The forms $a(\mathbf{k})$ and the operators $\mathcal{A}(\mathbf{k})$. Putting

$$
\mathfrak{H}=L_{2}\left(\Omega ; \mathbb{C}^{n}\right), \quad \mathfrak{H}_{*}=L_{2}\left(\Omega ; \mathbb{C}^{m}\right),
$$

we consider the closed operator

$$
\mathcal{X}(\mathbf{k}): \mathfrak{H} \rightarrow \mathfrak{H}_{*}, \quad \mathbf{k} \in \mathbb{R}^{d},
$$

defined on the domain

$$
\mathfrak{d}:=\operatorname{Dom} \mathcal{X}(\mathbf{k})=\left\{\mathbf{u} \in \mathfrak{H}: f \mathbf{u} \in \widetilde{H}^{1}\left(\Omega ; \mathbb{C}^{n}\right)\right\}
$$

by the formula

$$
\mathcal{X}(\mathbf{k})=h b(\mathbf{D}+\mathbf{k}) f, \quad \mathbf{k} \in \mathbb{R}^{d} .
$$

The selfadjoint operator

$$
\mathcal{A}(\mathbf{k}):=\mathcal{X}(\mathbf{k})^{*} \mathcal{X}(\mathbf{k}): \mathfrak{H} \rightarrow \mathfrak{H}, \quad \mathbf{k} \in \mathbb{R}^{d},
$$

is generated by the closed quadratic form

$$
a(\mathbf{k})[\mathbf{u}, \mathbf{u}]:=\|\mathcal{X}(\mathbf{k}) \mathbf{u}\|_{\mathfrak{H}_{*}}^{2}, \quad \mathbf{u} \in \mathfrak{d}, \quad \mathbf{k} \in \mathbb{R}^{d} .
$$

Using the Fourier series expansion (1.15), identity (1.17), and estimates (1.3), we see that

$$
\begin{aligned}
c_{0} \int_{\Omega}|(\mathbf{D}+\mathbf{k}) \mathbf{v}|^{2} d \mathbf{x} \leq a(\mathbf{k})[\mathbf{u}, \mathbf{u}] & \leq c_{1} \int_{\Omega}|(\mathbf{D}+\mathbf{k}) \mathbf{v}|^{2} d \mathbf{x}, \\
\mathbf{v} & =f \mathbf{u} \in \widetilde{H}^{1}\left(\Omega ; \mathbb{C}^{n}\right), \mathbf{k} \in \mathbb{R}^{d},
\end{aligned}
$$

where $c_{0}$ and $c_{1}$ are defined by (1.12). From (2.6) and the compactness of the embedding of $\widetilde{H}^{1}\left(\Omega ; \mathbb{C}^{n}\right)$ in $\mathfrak{H}$ it follows that the spectrum of $\mathcal{A}(\mathbf{k})$ is discrete. Observe also that the resolvent of the operator $\mathcal{A}(\mathbf{k})$ is compact and depends on $\mathbf{k} \in \mathbb{R}^{d}$ continuously (in the operator norm).

Let

$$
\mathfrak{N}:=\operatorname{Ker} \mathcal{A}(0)=\operatorname{Ker} \mathcal{X}(0) .
$$

Relations (2.6) with $\mathbf{k}=0$ show that

$$
\mathfrak{N}=\left\{\mathbf{u} \in L_{2}\left(\Omega ; \mathbb{C}^{n}\right): f \mathbf{u}=\mathbf{c} \in \mathbb{C}^{n}\right\}, \quad \operatorname{dim} \mathfrak{N}=n .
$$

We see that $\mathfrak{N}$ does not depend on the matrix $g$. 
2.2. The band functions. Let

$$
E_{1}(\mathbf{k}) \leq E_{2}(\mathbf{k}) \leq \cdots \leq E_{s}(\mathbf{k}) \leq \cdots, \quad \mathbf{k} \in \mathbb{R}^{d},
$$

be the consecutive eigenvalues of the operator $\mathcal{A}(\mathbf{k})$ (counted with multiplicities). The band functions $E_{s}(\mathbf{k})$ are continuous and $\widetilde{\Gamma}$-periodic.

In $\mathfrak{H}$, we consider the closed quadratic form

$$
a^{0}(\mathbf{k})[\mathbf{v}, \mathbf{v}]=\int_{\Omega}|(\mathbf{D}+\mathbf{k}) \mathbf{v}|^{2} d \mathbf{x}, \quad \mathbf{v} \in \widetilde{H}^{1}\left(\Omega ; \mathbb{C}^{n}\right)
$$

By (1.16), we have $a^{0}(\mathbf{k})[\mathbf{v}, \mathbf{v}]=\sum_{\mathbf{b} \in \widetilde{\Gamma}}|\mathbf{b}+\mathbf{k}|^{2}\left|\mathbf{v}_{\mathbf{b}}\right|^{2}$. This implies that the band functions $E_{j}^{0}(\mathbf{k})$ of the corresponding operator $\mathcal{A}^{0}(\mathbf{k})$ reduce to the numbers $|\mathbf{b}+\mathbf{k}|^{2}, \mathbf{b} \in \widetilde{\Gamma}$. Each of these eigenvalues is of multiplicity $n$. We list the properties of $E_{j}^{0}(\mathbf{k})$ necessary for what follows. They are quite obvious.

$1^{\circ} . E_{l}^{0}(\mathbf{k})=|\mathbf{k}|^{2}, l=1, \ldots, n, \mathbf{k} \in \operatorname{clos} \widetilde{\Omega}$.

$2^{\circ} . E_{1}^{0}(\mathbf{k}) \geq r^{2}, \mathbf{k} \in \operatorname{clos} \widetilde{\Omega} \backslash \mathcal{K}(r), 0<r \leq r_{0}$.

$3^{\circ} . E_{n+1}^{0}(\mathbf{k}) \geq r_{0}^{2}, \mathbf{k} \in \mathbb{R}^{d}$.

$4^{\circ} . E_{n+1}^{0}(0)=\min _{0 \neq \mathbf{b} \in \tilde{\Gamma}}|\mathbf{b}|^{2}=4 r_{0}^{2}$.

Now, we return to the functions (2.9). They coincide with the consecutive minima of the quotient $a(\mathbf{k})[\mathbf{u}, \mathbf{u}] /\|\mathbf{u}\|_{\mathfrak{H}}^{2}$. By (2.6), this quotient can be estimated from below as follows: for $\mathbf{u} \in \mathfrak{d}, \mathbf{v}=f \mathbf{u}$, we have

$$
\frac{a(\mathbf{k})[\mathbf{u}, \mathbf{u}]}{\|\mathbf{u}\|_{\mathfrak{H}}^{2}} \geq c_{0} \frac{a^{0}(\mathbf{k})[\mathbf{v}, \mathbf{v}]}{\|\mathbf{u}\|_{\mathfrak{H}}^{2}} \geq c_{0}\left\|f^{-1}\right\|_{L_{\infty}}^{-2} \frac{a^{0}(\mathbf{k})[\mathbf{v}, \mathbf{v}]}{\|\mathbf{v}\|_{\mathfrak{H}}^{2}} .
$$

Variational type arguments show that

$$
E_{j}(\mathbf{k}) \geq c_{*} E_{j}^{0}(\mathbf{k})
$$

where, in accordance with (1.12),

$$
c_{*}=\alpha_{0}\left\|f^{-1}\right\|_{L_{\infty}}^{-2}\left\|h^{-1}\right\|_{L_{\infty}}^{-2} .
$$

Combining estimates $(2.10)$ with the properties $1^{\circ}-4^{\circ}$ of the functions $E_{j}^{0}(\mathbf{k})$, we obtain the following:

$$
\begin{aligned}
E_{l}(\mathbf{k}) & \geq c_{*}|\mathbf{k}|^{2}, & & l=1, \ldots, n, \mathbf{k} \in \operatorname{clos} \widetilde{\Omega}, \\
E_{1}(\mathbf{k}) & \geq c_{*} r^{2}, & & \mathbf{k} \in \operatorname{clos} \widetilde{\Omega} \backslash \mathcal{K}(r), 0<r \leq r_{0}, \\
E_{n+1}(\mathbf{k}) & \geq c_{*} r_{0}^{2}, & & \mathbf{k} \in \mathbb{R}^{d}, \\
E_{n+1}(0) & \geq 4 c_{*} r_{0}^{2} . & &
\end{aligned}
$$

2.3. The direct integral for the operator $\mathcal{A}$. The operators $\mathcal{A}(\mathbf{k})$ allow us to partially diagonalize the operator $\mathcal{A}$ in the direct integral $\mathcal{H}$ (see (1.18)). Let us compare relations (1.6), (1.7), and (1.9), which define the form $a$ of the operator $\mathcal{A}$, with relations $(2.2),(2.3)$, and (2.5), which define the form $a(\mathbf{k})$ of the operator $\mathcal{A}(\mathbf{k})$. For $\mathbf{u} \in \operatorname{Dom} \mathcal{X}=$ $\operatorname{Dom} a$, we denote $\mathbf{v}=f \mathbf{u}$. From what was said in Subsection 1.3 about $\mathbf{v}$, we see directly that the following is true for $\mathbf{u}$. Let $\widetilde{\mathbf{u}}=\mathcal{U} \mathbf{u}, \mathbf{u} \in \operatorname{Dom} a$. Then

$$
\begin{aligned}
& \widetilde{\mathbf{u}}(\mathbf{k}, \cdot) \in \mathfrak{d} \quad \text { for a.e. } \mathbf{k} \in \mathbb{R}^{d}, \\
& a[\mathbf{u}, \mathbf{u}]=\int_{\widetilde{\Omega}} a(\mathbf{k})[\widetilde{\mathbf{u}}(\mathbf{k}, \cdot), \widetilde{\mathbf{u}}(\mathbf{k}, \cdot)] d \mathbf{k} .
\end{aligned}
$$

Conversely, if $\widetilde{\mathbf{u}} \in \mathcal{H}$ satisfies (2.15) and the integral in (2.16) is finite, then $\mathbf{u} \in \operatorname{Dom} a$ and (2.16) is valid. The above arguments show that, in the direct integral $\mathcal{H}$, the operator $\mathcal{A}$ 
(see (1.8)) turns into multiplication by the operator-valued function $\mathcal{A}(\mathbf{k}), \mathbf{k} \in \widetilde{\Omega}$, defined by (2.4). All this can be expressed briefly by the formula

$$
\mathcal{U} \mathcal{A} \mathcal{U}^{-1}=\int_{\widetilde{\Omega}} \oplus \mathcal{A}(\mathbf{k}) d \mathbf{k} .
$$

From (2.17) it follows that the spectrum $\sigma(\mathcal{A})$ is a union of segments (spectral bands) that are the ranges of the band functions (2.9). Relations (2.7) and (2.8) imply that

$$
\min _{\mathbf{k} \in \mathbb{R}^{d}} E_{j}(\mathbf{k})=E_{j}(0)=0, \quad j=1, \ldots, n .
$$

Consequently, the lower edge of the spectrum of $\mathcal{A}$ coincides with the point $\lambda=0$ : $\inf \sigma(\mathcal{A})=0$.

§3. Incorporation of the operators $\mathcal{A}(\mathbf{k})$ into the pattern of $\S 1$, Chapter 1

3.1. Concordance of notions. For $d>1$, the operators $\mathcal{A}(\mathbf{k})$ depend on the multidimensional parameter $\mathbf{k}$. In this case, the analytic perturbation theory gives satisfactory results only for simple eigenvalues. The role of the unperturbed operator $A_{0}$ is played by $\mathcal{A}(0)$. By (2.7) and (2.8), we now have $\operatorname{dim} \mathfrak{N}=n \geq 1$, i.e., for $n>1$, the eigenvalue $\lambda=0$ for $\mathcal{A}(0)$ is multiple. To avoid this difficulty, for $\mathbf{k} \in \mathbb{R}^{d}$ we put $\mathbf{k}=t \boldsymbol{\theta}, t=|\mathbf{k}|,|\boldsymbol{\theta}|=1$, and view $t$ as the perturbation parameter. At the same time, all the constructions will depend on the additional parameter $\boldsymbol{\theta}$. This dependence will often be reflected in the notation.

We shall apply the method described in $\S 1$ of Chapter 1 ; we put

$$
\mathfrak{H}=L_{2}\left(\Omega ; \mathbb{C}^{n}\right), \quad \mathfrak{H}_{*}=L_{2}\left(\Omega ; \mathbb{C}^{m}\right),
$$

which coincides with (2.1). Next,

$$
\begin{aligned}
X(t) & =X(t, \boldsymbol{\theta})=\mathcal{X}(t \boldsymbol{\theta})=h b(\mathbf{D}+t \boldsymbol{\theta}) f, \\
X_{0} & =\mathcal{X}(0)=h b(\mathbf{D}) f, \quad \operatorname{Dom} X_{0}=\mathfrak{d}, \\
X_{1} & =X_{1}(\boldsymbol{\theta})=h b(\boldsymbol{\theta}) f, \\
X(t) & =X_{0}+t X_{1},
\end{aligned}
$$

and, in (3.3), we may assume that $t \in \mathbb{R}$. Finally,

$$
A(t)=A(t, \boldsymbol{\theta})=\mathcal{A}(t \boldsymbol{\theta})
$$

and, in accordance with (2.7) and (2.8),

$$
\mathfrak{N}=\operatorname{Ker} X_{0}=\operatorname{Ker} \mathcal{X}(0), \quad \operatorname{dim} \mathfrak{N}=n .
$$

Relation (1.1) guarantees that $n \leq n_{*}$. Moreover, since

$$
\mathfrak{N}_{*}=\operatorname{Ker} X_{0}^{*}=\left\{\mathbf{q} \in L_{2}\left(\Omega ; \mathbb{C}^{m}\right): h^{*} \mathbf{q} \in \widetilde{H}^{1}\left(\Omega ; \mathbb{C}^{m}\right), b(\mathbf{D})^{*} h(\mathbf{x})^{*} \mathbf{q}=0\right\},
$$

we see that, under condition (1.1), the following alternative realizes for $n_{*}=\operatorname{dim} \mathfrak{N}_{*}$ : either $n_{*}=\infty$ (if $m>n$ ), or $n_{*}=n$ (if $m=n$ ). By (2.14), instead of the precise value $d^{0}=E_{n+1}(0)$ we take

$$
d^{0}=4 c_{*} r_{0}^{2}
$$

(possibly, a larger value could be taken). Here $c_{*}$ is the constant (2.11). In Subsection 1.1.1, it was required to choose $\delta<d^{0} / 8$. Recalling (3.6), we fix $\delta$ so that

$$
\delta<c_{*} r_{0}^{2} / 2=2^{-1} r_{0}^{2} \alpha_{0}\left\|f^{-1}\right\|_{L_{\infty}}^{-2}\left\|h^{-1}\right\|_{L_{\infty}}^{-2} .
$$


Next, the estimate $\left\|X_{1}(\boldsymbol{\theta})\right\| \leq \alpha_{1}^{1 / 2}\|f\|_{L_{\infty}}\|h\|_{L_{\infty}}$ allows us to choose $t^{0}(\delta)$ (see (1.1.3)) equal not to $\delta^{1 / 2}\left\|X_{1}(\theta)\right\|^{-1}$, but to a smaller number independent of $\theta$. Namely, we put

$$
t^{0}(\delta)=\delta^{1 / 2} \alpha_{1}^{-1 / 2}\|f\|_{L_{\infty}}^{-1}\|h\|_{L_{\infty}}^{-1} .
$$

Observe that $t^{0}(\delta)<r_{0} / \sqrt{2}$ by (3.7) and (3.8). Thus,

$$
\left\{\mathbf{k} \in \widetilde{\Omega}:|\mathbf{k}|=t \leq t^{0}(\delta)\right\} \subset \mathcal{K}\left(2^{-1 / 2} r_{0}\right) \subset \widetilde{\Omega} .
$$

In what follows, we always assume that $c_{*}$ and $t^{0}(\delta)$ are defined by (2.11) and (3.8).

3.2. Nondegeneracy of the germ of the family $A(t, \boldsymbol{\theta})$. The analytic (in $t$ ) branches of the eigenvalues $\lambda_{l}(t, \theta)$ (see (1.1.28) and (1.1.29)) and the branches of the eigenvectors $\varphi_{l}(t, \boldsymbol{\theta}), l=1, \ldots, n,|t| \leq t^{0}(\delta)$, depend on $\boldsymbol{\theta}$. The first band functions $E_{l}(t \boldsymbol{\theta}), l=$ $1, \ldots, n$ (see $(2.9)$ ), coincide with $\lambda_{l}(t, \boldsymbol{\theta})$ only partially, because the analytic branches $\lambda_{l}$ are not enumerated in nondecreasing order. At the same time, from (2.12) it follows that

$$
\lambda_{l}(t, \boldsymbol{\theta}) \geq c_{*} t^{2}, \quad l=1, \ldots, n, \quad t \in\left[0, t^{0}(\delta)\right] .
$$

It is essential that $c_{*}$ and $t^{0}(\delta)$ in (3.9) do not depend on $\theta$. Inequalities (3.9) show that the germ $S(\boldsymbol{\theta})$ of the family $A(t, \boldsymbol{\theta})$ (see (3.4)) is nondegenerate uniformly in $\boldsymbol{\theta}$.

\section{Chapter 3. Effective characteristics near the lower edge OF THE SPECTRUM}

In this chapter, we consider the spectral germ $S(\boldsymbol{\theta})$ of the operator family $A(t, \boldsymbol{\theta})$ (see (2.3.4)). We introduce a family that is threshold equivalent to the given one simultaneously for all $\boldsymbol{\theta}$ and is such that the corresponding matrix $g=h^{*} h$ for this family is constant. This constant matrix is called the effective matrix (notation: $g_{\mathrm{eff}}$ ) for the initial periodic matrix $g$. We discuss the question about the uniqueness of $g_{\text {eff }}$; herewith, we correct a wrong statement concerning this question that occurred in [BSu2]. We distinguish the main effective matrix $g^{0}$ and discuss its properties. We introduce the effective DO for the DO $\mathcal{A}$ (see (2.1.10)). The effective DO is of the same type as $\mathcal{A}$, but with $g$ replaced by $g_{\text {eff }}$. On the basis of $\S 5$ in Chapter 1 , we study the behavior of the resolvent $\left(\mathcal{A}+\varepsilon^{2} I\right)^{-1}$ as $\varepsilon \rightarrow 0$.

Below, we shall use the following notation. Let $\phi(\mathbf{x})$ be a $\Gamma$-periodic matrix-valued function such that $\phi \in L_{1, \text { loc }}\left(\mathbb{R}^{d}\right)$. We put

$$
\bar{\phi}=|\Omega|^{-1} \int_{\Omega} \phi(\mathbf{x}) d \mathbf{x} .
$$

If, moreover, the matrix $\phi$ is square, nonsingular, and such that $\phi^{-1} \in L_{1, \text { loc }}\left(\mathbb{R}^{d}\right)$, we put

$$
\underline{\phi}=\left(|\Omega|^{-1} \int_{\Omega} \phi(\mathbf{x})^{-1} d \mathbf{x}\right)^{-1}
$$

Observe that, if $\phi(\mathbf{x})>0$, then we always have $\underline{\phi} \leq \bar{\phi}$, and equality occurs only if $\phi$ is a constant matrix.

\section{§1. Effective matrices And the EFfective DO}

First, we need to construct the operators $R=R(\boldsymbol{\theta})$ and $S=S(\boldsymbol{\theta})$ for the family $A(t, \boldsymbol{\theta})$. It is convenient to start with the case where $f=\mathbf{1}_{n}$, and then proceed to the general case on the basis of Subsection 1.1.5. In the case where $f=\mathbf{1}_{n}$, we agree to mark all the corresponding objects by "へ". 
1.1. The operator $\widehat{R}(\boldsymbol{\theta})$. We shall use the notation (2.3.1). In accordance with (2.2.8),

$$
\widehat{\mathfrak{N}}=\left\{\mathbf{u} \in \mathfrak{H}: \mathbf{u}=\mathbf{c} \in \mathbb{C}^{n}\right\}, \quad \widehat{n}=n .
$$

Since $\mathfrak{N}_{*}$ does not depend on $f$ (see (2.3.5)), we have $\widehat{\mathfrak{N}}_{*}=\mathfrak{N}_{*}$. On the other hand, $\mathfrak{N}_{*}$ depends on $g$, and, moreover, on $h$.

Let

$$
\mathfrak{M}=\left\{\mathbf{q} \in \mathfrak{H}_{*}: \mathbf{q}=\mathbf{C} \in \mathbb{C}^{m}\right\}
$$

be the subspace of constant vector-valued functions. By Subsection 1.1.2, for every $\mathbf{C} \in \mathfrak{M}$ there exists a unique element $\mathbf{v} \in \widetilde{H}^{1}\left(\Omega ; \mathbb{C}^{n}\right)$ such that $\int_{\Omega} \mathbf{v} d \mathbf{x}=0$ and (see (1.1.7) with $z=-h \mathbf{C}$ )

$$
(g b(\mathbf{D}) \mathbf{v}, b(\mathbf{D}) \mathbf{w})_{\mathfrak{H}_{*}}=-(g \mathbf{C}, b(\mathbf{D}) \mathbf{w})_{\mathfrak{H}_{*}}, \quad \mathbf{w} \in \widetilde{H}^{1}\left(\Omega ; \mathbb{C}^{n}\right) .
$$

We put

$$
B \mathbf{C}=h(b(\mathbf{D}) \mathbf{v}+\mathbf{C}) \in \mathfrak{N}_{*} \subset \mathfrak{H}_{*} .
$$

Obviously, the operator $B: \mathfrak{M} \rightarrow \mathfrak{N}_{*}$ is independent of $\mathbf{k}=t \boldsymbol{\theta}$. Observe the identity $\int_{\Omega} h^{-1} B \mathbf{C} d \mathbf{x}=|\Omega| \mathbf{C}$. This implies that $\operatorname{Ker} B=\{0\}$, whence $\operatorname{rank} B=m$. Now, let $\mathbf{c} \in \widehat{\mathfrak{N}}$ and $\mathbf{C}=b(\boldsymbol{\theta}) \mathbf{c}$. Then (see (1.1.7)-(1.1.10)) $\mathbf{v}$ plays the role of $\widehat{\varphi}(\mathbf{c})$ and $\widehat{R}(\boldsymbol{\theta}) \mathbf{c}=B(b(\boldsymbol{\theta}) \mathbf{c})$. Thus,

$$
\widehat{R}(\boldsymbol{\theta}): \widehat{\mathfrak{N}} \rightarrow \mathfrak{N}_{*}, \quad \widehat{R}(\boldsymbol{\theta})=B b(\boldsymbol{\theta}), \quad \boldsymbol{\theta} \in S S^{d-1} .
$$

By condition (2.1.2), from (1.5) and the relation $\operatorname{Ker} B=\{0\}$ it follows that $\operatorname{Ker} \widehat{R}(\boldsymbol{\theta})=$ $\{0\}$, or, equivalently,

$$
\operatorname{rank} \widehat{R}(\boldsymbol{\theta})=n .
$$

We emphasize that, in the preceding calculations, the operators $b(\xi), B$, and $\widehat{R}(\boldsymbol{\theta})$ were represented as matrices written in some fixed bases (see Subsection 2.1.1). However, the final relations (e.g., (1.5)) have invariant meaning. The same concerns all that follows; in particular, we do not distinguish between the germ $\widehat{S}(\boldsymbol{\theta})$ and the matrix corresponding to this germ in a fixed basis.

1.2. The operator $\widehat{S}(\boldsymbol{\theta})$. Effective matrices. In the sequel, it is convenient to indicate the dependence of the objects on the matrix $g$ (for a fixed matrix $b(\xi)$ ) in the notation. For instance, we shall write $\widehat{A}(t, \boldsymbol{\theta} ; g), \widehat{R}(\boldsymbol{\theta} ; g), \widehat{S}(\boldsymbol{\theta} ; g)$, etc. By (1.5), it is clear that the operator

$$
\widehat{S}(\boldsymbol{\theta} ; g)=\widehat{R}(\boldsymbol{\theta} ; g)^{*} \widehat{R}(\boldsymbol{\theta} ; g): \widehat{\mathfrak{N}} \rightarrow \widehat{\mathfrak{N}}
$$

(the germ of the family $\widehat{A}(t, \boldsymbol{\theta} ; g)$ ) satisfies the relation $\widehat{S}(\boldsymbol{\theta} ; g)=b(\boldsymbol{\theta})^{*}\left(B^{*} B\right) b(\boldsymbol{\theta})$. From (1.6) and (1.7) (see Subsection 2.3.2) it follows that the germ $\widehat{S}(\boldsymbol{\theta} ; g)$ is nondegenerate. In a fixed basis $\mathbf{e}_{1}, \ldots, \mathbf{e}_{m}$, the operator $B^{*} B: \mathfrak{M} \rightarrow \mathfrak{M}$ is represented by a constant (and independent of $\boldsymbol{\theta}$ ) positive matrix $g^{0}$. Thus,

$$
\widehat{S}(\boldsymbol{\theta} ; g)=b(\boldsymbol{\theta})^{*} g^{0} b(\boldsymbol{\theta}), \quad g^{0}=B^{*} B .
$$

Now, along with $\widehat{A}(t, \theta ; g)$, we consider the family $\widehat{A}\left(t, \theta ; g^{0}\right)$, where the matrix $g^{0}$ is associated with the matrix-valued function $g$ as described above. We may assume that the matrix $g^{0}$ is factorized somehow: $g^{0}=\left(h^{0}\right)^{*} h^{0}$; the final results do not depend on the choice of this factorization. If $g=g^{0}$, relation (1.3) yields $\mathbf{v}=0$. Then $\widehat{R}\left(\boldsymbol{\theta} ; g^{0}\right)=h^{0} b(\boldsymbol{\theta})$ and $\widehat{S}\left(\boldsymbol{\theta} ; g^{0}\right)=b(\boldsymbol{\theta})^{*} g^{0} b(\boldsymbol{\theta})$, i.e., $\left(g^{0}\right)^{0}=g^{0}$. Thus,

$$
\widehat{S}(\boldsymbol{\theta} ; g)=\widehat{S}\left(\boldsymbol{\theta} ; g^{0}\right)=b(\boldsymbol{\theta})^{*} g^{0} b(\boldsymbol{\theta}), \quad \boldsymbol{\theta} \in S S^{d-1} .
$$


In particular, (1.9) shows that the families $\widehat{A}(t, \boldsymbol{\theta} ; g)$ and $\widehat{A}\left(t, \boldsymbol{\theta} ; g^{0}\right)$ are threshold equivalent. We put

$$
\widehat{S}\left(\mathbf{k} ; g^{0}\right):=t^{2} \widehat{S}\left(\boldsymbol{\theta} ; g^{0}\right)=b(\mathbf{k})^{*} g^{0} b(\mathbf{k}), \quad \mathbf{k}=t \boldsymbol{\theta} \in \mathbb{R}^{d} .
$$

Then $\widehat{S}\left(\mathbf{k} ; g^{0}\right)$ is the symbol of the DO

$$
\widehat{\mathcal{A}}^{0}:=b(\mathbf{D})^{*} g^{0} b(\mathbf{D})
$$

with constant coefficients.

Definition 1.1. A constant positive $(m \times m)$-matrix $g_{\text {eff }}$ is called an effective matrix for the operator family $\widehat{A}(t, \boldsymbol{\theta} ; g)$ if $\widehat{S}(\boldsymbol{\theta} ; g)=\widehat{S}\left(\boldsymbol{\theta} ; g_{\text {eff }}\right)$ for all $\boldsymbol{\theta} \in S S^{d-1}$.

If $g_{\text {eff }}$ is an effective matrix for $g$, then

$$
\widehat{S}(\boldsymbol{\theta} ; g)=\widehat{S}\left(\boldsymbol{\theta} ; g_{\mathrm{eff}}\right)=b(\boldsymbol{\theta})^{*} g_{\mathrm{eff}} b(\boldsymbol{\theta}), \quad \boldsymbol{\theta} \in S S^{d-1} .
$$

Relations (1.8) and (1.9) imply the following statement.

Proposition 1.2. For the operator family $\widehat{A}(t, \theta ; g)$, there exists at least one effective matrix. Namely, the matrix $g^{0}$ corresponding to the operator $B^{*} B$ is effective.

Definition 1.3. The matrix $g^{0}$ is called the main effective matrix for the family $\widehat{A}(t, \boldsymbol{\theta} ; g)$.

Definition 1.4. The operator (1.10) $\widehat{\mathcal{A}}^{0}$ is called the effective $D O$ for the operator $\widehat{\mathcal{A}}=b(\mathbf{D})^{*} g b(\mathbf{D})$ acting in $L_{2}\left(\mathbb{R}^{d} ; \mathbb{C}^{n}\right)$.

We mention at once that, by (1.11), $\widehat{S}\left(\boldsymbol{\theta} ; g_{\text {eff }}\right)$ coincides with $\widehat{S}\left(\boldsymbol{\theta} ; g^{0}\right)$, and then $\widehat{S}\left(\mathbf{k} ; g_{\text {eff }}\right)=t^{2} \widehat{S}\left(\boldsymbol{\theta} ; g_{\text {eff }}\right)=\widehat{S}\left(\mathbf{k} ; g^{0}\right)$. This implies that the effective operator $\widehat{\mathcal{A}}^{0}$ for $\widehat{\mathcal{A}}=b(\mathbf{D})^{*} g b(\mathbf{D})$ is defined uniquely. In other words, $\widehat{\mathcal{A}}^{0}$ will not change if in (1.10) we replace $g^{0}$ by any other matrix $g_{\text {eff }}$.

Let $\mathbf{v}_{j}$ be the solution of problem (1.3) with $\mathbf{C}=\mathbf{e}_{j}, j=1, \ldots, m$. We put $\mathbf{u}_{j}:=$ $B \mathbf{e}_{j}=h\left(b(\mathbf{D}) \mathbf{v}_{j}+\mathbf{e}_{j}\right)$. Then $|\Omega| g^{0}$ is a Gram matrix, i.e.,

$$
g^{0}=|\Omega|^{-1}\left\{\left(\mathbf{u}_{j}, \mathbf{u}_{l}\right)_{\mathfrak{H}_{*}}\right\}, \quad j, l=1, \ldots, m .
$$

If all the entries of the matrices $g(\mathbf{x})$ and $b(\xi)$ are real-valued, then the solutions $\mathbf{v}_{j}$ are purely imaginary, and the vector-valued functions $\mathbf{u}_{j}$ are real. In this case, from (1.12) it follows that the matrix $g^{0}$ also has real entries. Then it is natural (but not necessary) to require that all other effective matrices have real entries.

We supplement the representations $g^{0}=B^{*} B$ and (1.12) with yet another useful representation for the main effective matrix $g^{0}$. Usually, this representation is viewed as initial in homogenization theory.

We recall the notation (2.3.2), (2.3.3), and (1.2). For $\mathbf{C} \in \mathfrak{M}$, we rewrite (1.4) as

$$
h \mathbf{C}=B \mathbf{C}-h b(\mathbf{D}) \mathbf{v}, \quad \mathbf{C} \in \mathfrak{M} .
$$

Since $B \mathbf{C} \in \mathfrak{N}_{*}=\operatorname{Ker} X_{0}^{*}$ and $h b(\mathbf{D}) \mathbf{v} \in \operatorname{Ran} X_{0}$, the summands on the right are orthogonal to each other in $\mathfrak{H}_{*}$. Consequently,

$$
\|B \mathbf{C}\|_{\mathfrak{H}_{*}}^{2}=(B \mathbf{C}, h \mathbf{C})_{\mathfrak{H}_{*}}=\left(h^{*} B \mathbf{C}, \mathbf{C}\right)_{\mathfrak{H}_{*}}=(g(\mathbf{C}+b(\mathbf{D}) \mathbf{v}), \mathbf{C})_{\mathfrak{H}_{*}},
$$

whence

$$
|\Omega|\left\langle g^{0} \mathbf{C}, \mathbf{C}\right\rangle_{\mathbb{C}^{m}}=\left\langle\int_{\Omega} g(b(\mathbf{D}) \mathbf{v}+\mathbf{C}) d \mathbf{x}, \mathbf{C}\right\rangle_{\mathbb{C}^{m}} .
$$

Thus, for $g^{0}$ we have

$$
g^{0} \mathbf{C}=|\Omega|^{-1} \int_{\Omega} g(\mathbf{x})(b(\mathbf{D}) \mathbf{v}+\mathbf{C}) d \mathbf{x}, \quad \mathbf{C} \in \mathfrak{M},
$$

where $\mathbf{v}$ is defined by (1.3). 
1.3. On the uniqueness of an effective matrix. Comparing (1.9) and (1.11) and passing to quadratic forms, we obtain

$$
\left\langle g^{0} b(\boldsymbol{\theta}) \zeta, b(\boldsymbol{\theta}) \zeta\right\rangle=\left\langle g_{\mathrm{eff}} b(\boldsymbol{\theta}) \zeta, b(\boldsymbol{\theta}) \zeta\right\rangle, \quad \zeta \in \mathbb{C}^{n}, \boldsymbol{\theta} \in S S^{d-1} .
$$

It follows that the matrix $g_{\text {eff }}=g^{0}$ is unique if and only if

$$
\operatorname{clos} \bigcup_{\boldsymbol{\theta}} \operatorname{Ran} b(\boldsymbol{\theta})=\mathbb{C}^{m} \text {. }
$$

If the matrices $g(\mathbf{x})$ and $b(\boldsymbol{\theta})$ (and then also $g^{0}$ ) have all entries real, then the uniqueness of the real matrix $g_{\text {eff }}$ is ensured by the condition

$$
\operatorname{clos} \bigcup_{\boldsymbol{\theta}}\left\{b(\boldsymbol{\theta}) \mathbb{R}^{n}\right\}=\mathbb{R}^{m} .
$$

In [BSu2, a false algebraic criterion of the validity of (1.15) was formulated. In fact, the uniqueness of $g_{\text {eff }}$ is a comparatively rare property. This is related to the fact that one and the same operator $\widehat{\mathcal{A}}=b(\mathbf{D})^{*} g(\mathbf{x}) b(\mathbf{D})$ can be described by different matrices $g(\mathbf{x})$.

Note that, by (2.1.2), conditions (1.15) and (1.16) are valid a fortiori if $m=n$. So, if $m=n$, then an effective matrix is unique. Next, it is easily seen that, in the real-valued case, (1.16) is valid if $n=1$ and $m=d$. This case corresponds to a real scalar elliptic operator $\widehat{\mathcal{A}}$. Here the requirement that $g(\mathbf{x})$ have all entries real is essential. For instance, if $n=1, m=d=2$, and $b(\xi)=\mathbf{e}_{1} \xi^{1}+\mathbf{e}_{2} \xi^{2}$, then any matrix of the form $g^{0}+i \alpha \check{g}$ will be effective. Here $\check{g}=\left(\begin{array}{cc}0 & 1 \\ -1 & 0\end{array}\right)$ and $\alpha \in \mathbb{R}$ is sufficiently small. If $n>1$, the uniqueness of $g_{\text {eff }}$ may be violated even in the real-valued case.

Since the effective DO $\widehat{\mathcal{A}}^{0}$ does not depend on the choice of $g_{\text {eff }}$, the nonuniqueness of the latter matrix is not very essential. In what follows, the main attention will be paid to the main effective matrix $g^{0}$.

1.4. Estimates for the matrix $g^{0}$. The Voight-Reuss bracketing. In accordance with $(0.1)$ and $(0.2)$, for the matrix $g(\mathbf{x})$ we put

$$
\bar{g}=|\Omega|^{-1} \int_{\Omega} g(\mathbf{x}) d \mathbf{x}, \quad \underline{g}=\left(|\Omega|^{-1} \int_{\Omega} g(\mathbf{x})^{-1} d \mathbf{x}\right)^{-1} .
$$

Since the summands on the right-hand side of (1.13) are orthogonal to each other, we have

$$
\|B \mathbf{C}\|_{\mathfrak{H}_{*}}^{2} \leq\|h \mathbf{C}\|_{\mathfrak{H}_{*}}^{2} .
$$

It is easily seen that (1.18) is equivalent to the relation $\left\langle B^{*} B \mathbf{C}, \mathbf{C}\right\rangle_{\mathbb{C}^{m}} \leq\langle\bar{g} \mathbf{C}, \mathbf{C}\rangle_{\mathbb{C}^{m}}$, and since $g^{0}=B^{*} B$ (see (1.8)), we obtain the upper estimate $g^{0} \leq \bar{g}$.

In order to prove the lower estimate, we observe that, by $(2.3 .5), \mathfrak{P}:=\left(h^{*}\right)^{-1} \mathfrak{M} \subset$ $\mathfrak{N}_{*} \subset \mathfrak{H}_{*}$. We put

$$
\Pi \mathbf{w}=|\Omega|^{-1}\left(h^{*}\right)^{-1} \underline{g} \int_{\Omega} h^{-1} \mathbf{w} d \mathbf{x}, \quad \mathbf{w} \in \mathfrak{H}_{*} .
$$

An elementary argument shows that $\Pi \mathbf{w} \in \mathfrak{P},\left.\Pi\right|_{\mathfrak{P}}=I_{\mathfrak{P}}$, and

$$
(\Pi \mathbf{w}, \mathbf{w})_{\mathfrak{H}_{*}}=|\Omega|^{-1}\left\langle\underline{g} \mathbf{C}_{\mathbf{w}}, \mathbf{C}_{\mathbf{w}}\right\rangle_{\mathbb{C}^{m}}, \mathbf{C}_{\mathbf{w}}=\int_{\Omega} h^{-1} \mathbf{w} d \mathbf{x}, \quad \mathbf{w} \in \mathfrak{H}_{*} .
$$

This means that $\Pi$ is the orthogonal projection of $\mathfrak{H}_{*}$ onto the subspace $\mathfrak{P}$. Now, we apply the projection $\Pi$ to (1.13). Since $h b(\mathbf{D}) \mathbf{v} \in \mathfrak{N}_{*}^{\perp}$, we obtain $\Pi B \mathbf{C}=\Pi h \mathbf{C}$. Putting $\mathbf{w}=h \mathbf{C}$ in (1.19), we arrive at

$$
\|\Pi h \mathbf{C}\|_{\mathfrak{H}_{*}}^{2}=|\Omega|\langle\underline{g} \mathbf{C}, \mathbf{C}\rangle_{\mathbb{C}^{m}} .
$$


Consequently,

$$
|\Omega|\left\langle g^{0} \mathbf{C}, \mathbf{C}\right\rangle_{\mathbb{C}^{m}}=\|B \mathbf{C}\|_{\mathfrak{H}_{*}}^{2} \geq\|\Pi B \mathbf{C}\|_{\mathfrak{H}_{*}}^{2}=\|\Pi h \mathbf{C}\|_{\mathfrak{H}_{*}}^{2}=|\Omega|\langle\underline{g} \mathbf{C}, \mathbf{C}\rangle_{\mathbb{C}^{m}}
$$

Thus, we have proved the lower estimate: $g^{0} \geq \underline{g}$.

In the case where $m=n$, we can say more. Then $\operatorname{dim} \mathfrak{N}_{*}=n_{*}=n=m$. Since $\operatorname{dim} \mathfrak{P}=m$, we have $\mathfrak{N}_{*}=\mathfrak{P}$. Since $B \mathbf{C} \in \mathfrak{N}_{*}$, we have equality throughout in (1.20). As a result, we see that if $m=n$, then we always have $g^{0}=\underline{g}$.

Summarizing, we obtain the following statement.

Theorem 1.5. For the main effective matrix $g^{0}$ of the family $\widehat{A}(t, \boldsymbol{\theta} ; g)$, we have

$$
\underline{g} \leq g^{0} \leq \bar{g}
$$

where the constant matrices $\bar{g}$ and $\underline{g}$ are defined by (1.17). If $m=n$, then the (unique) effective matrix $g^{0}$ is equal to $\underline{g}$ :

$$
g^{0}=\underline{g}, \quad m=n .
$$

Now, we find conditions under which one of the inequalities in (1.21) becomes an identity. We start with the case where $g^{0}=\bar{g}$. This is equivalent to the fact that equality occurs in (1.18) for any $\mathbf{C}$. By (1.13), the latter means that $h b(\mathbf{D}) \mathbf{v}=0$, where $\mathbf{v}$ satisfies (1.3). But then (1.3) reduces to the relation $b(\mathbf{D})^{*} g(\mathbf{x}) \mathbf{C}=0, \mathbf{C} \in \mathbb{C}^{m}$. Let $\mathbf{g}_{k}(\mathbf{x}), k=1, \ldots, m$, be the columns of the matrix $g(\mathbf{x})$. As a result, we obtain the following statement.

Proposition 1.6. The identity $g^{0}=\bar{g}$ is equivalent to the relations

$$
b(\mathbf{D})^{*} \mathbf{g}_{k}(\mathbf{x})=0, \quad k=1, \ldots, m .
$$

We note that (1.23) contains $m n$ differential conditions imposed on the $m^{2}$ coefficients of the matrix $g$.

In the analysis of the case where $g^{0}=\underline{g}$ we may assume that $m>n$. By (1.20), the relation $g^{0}=g$ is equivalent to the fact that $B \mathbf{C} \in \mathfrak{P}, \mathbf{C} \in \mathfrak{M}$. In other words, for any $\mathbf{C} \in \mathbb{C}^{m}$ there exists $\mathbf{C}_{*} \in \mathbb{C}^{m}$ such that $B \mathbf{C}=\left(h^{*}\right)^{-1} \mathbf{C}_{*}$. By (1.4), this means that

$$
g^{-1} \mathbf{C}_{*}=\mathbf{C}+b(\mathbf{D}) \mathbf{v}
$$

Integrating (1.24), we obtain $\mathbf{C}=\underline{g}^{-1} \mathbf{C}_{*}$. Putting $\mathbf{C}_{*}=\mathbf{e}_{k}$, we can rewrite (1.24) in terms of the columns $\mathbf{l}_{k}(\mathbf{x})$ of the matrix $g(\mathbf{x})^{-1}$. We have proved the following statement.

Proposition 1.7. The identity $g^{0}=\underline{g}$ is equivalent to the relations

$$
\mathbf{l}_{k}(\mathbf{x})=\mathbf{l}_{k}^{0}+b(\mathbf{D}) \mathbf{v}_{k}, \quad \mathbf{v}_{k} \in \widetilde{H}^{1}\left(\Omega ; \mathbb{C}^{n}\right), \mathbf{l}_{k}^{0} \in \mathbb{C}^{m}, \quad k=1, \ldots, m,
$$

for the columns $\mathbf{l}_{k}(\mathbf{x})$ of the matrix $g(\mathbf{x})^{-1}$.

Moreover, (1.25) automatically implies that $\mathbf{l}_{k}^{0}=\underline{g}^{-1} \mathbf{e}_{k}$ and $\mathbf{v}_{k}$ satisfies (1.3) with $\mathbf{C}=\mathbf{l}_{k}^{0}$.

Let us comment on relations (1.21), (1.23), and (1.25). In specific cases (the acoustic operator, the operator of elasticity theory, etc.), estimates (1.21) are well known in homogenization theory; they are called the Voigt-Reuss bracketing (see, e.g., [ZhKO]). Our proof of (1.21) is parallel to the proof of the estimates for the germ $S$ given in Subsection 1.1.4 in abstract terms. By (1.9), estimates (1.21) imply the following inequalities for germs:

$$
\widehat{S}(\boldsymbol{\theta} ; \underline{g}) \leq \widehat{S}\left(\boldsymbol{\theta} ; g^{0}\right)=\widehat{S}(\boldsymbol{\theta} ; g) \leq \widehat{S}(\boldsymbol{\theta} ; \bar{g})
$$

We can return from (1.26) to (1.21), but only under the additional condition (1.15). Precisely under this condition estimates (1.21) were proved in [BSu2 for the operators $b(\mathbf{D})^{*} g(\mathbf{x}) b(\mathbf{D})$. Relation (1.22) with $n>1$ was distinguished for the first time in [BSu2]. 
For the acoustic operator, i.e., in the case where $n=1, m=d$, and $b(\mathbf{D})=\mathbf{D}$, the results of Propositions 1.6 and 1.7 have been known (see [ZhKO]). In this case relations (1.23) mean that the columns $\mathbf{g}_{k}(\mathbf{x})$ are solenoidal, and relations (1.25) mean that the columns $\mathbf{l}_{k}(\mathbf{x})$ are potential (up to a constant summand - this will be meant in what follows).

1.5. The case where $f \neq \mathbf{1}_{n}$. We return to the operators $\mathcal{A}$ of the general form (2.1.10) and to the corresponding families $A(t, \boldsymbol{\theta})$ of the form (2.3.4). As before, we shall indicate the dependence on the matrix $g$ (but not on the matrices $f, b$ ) in the notation. The upper mark " $\leadsto$ " is reserved for denoting the objects that correspond to the case of $f=\mathbf{1}_{n}$, with the same $b$ and $g$.

We use the pattern of Subsection 1.1.5. Now we have

$$
\widehat{\mathfrak{H}}=\mathfrak{H}=L_{2}\left(\Omega ; \mathbb{C}^{n}\right),
$$

and the role of the isomorphism $M$ is played by the operator of multiplication by the matrix-valued function $f$. The kernel $\mathfrak{N}=\operatorname{Ker} X_{0}$ is defined by (2.2.8):

$$
\mathfrak{N}=\left\{\mathbf{u} \in \mathfrak{H}: f \mathbf{u}=\mathbf{c} \in \mathbb{C}^{n}\right\}
$$

it does not depend on $b$ and $g$. By (1.1.21), we have $R(\boldsymbol{\theta} ; g)=\left.\widehat{R}(\boldsymbol{\theta} ; g) f\right|_{\mathfrak{N}}$. From (1.1.23) it follows that

$$
S(\boldsymbol{\theta} ; g)=\left.P f^{*} \widehat{S}(\boldsymbol{\theta} ; g) f\right|_{\mathfrak{N}},
$$

where $P$ is the orthogonal projection of $\mathfrak{H}$ onto $\mathfrak{N}$. Definition 1.1 is carried over to the general case of the family $A(t, \theta ; g)$.

Definition 1.8. A constant positive $(m \times m)$-matrix $g_{\text {eff }}$ is called an effective matrix for the operator family $A(t, \boldsymbol{\theta} ; g)$ if $S(\boldsymbol{\theta} ; g)=S\left(\boldsymbol{\theta} ; g_{\mathrm{eff}}\right)$ for all $\boldsymbol{\theta} \in S S^{d-1}$.

The following proposition is a direct consequence of (1.27).

Proposition 1.9. The matrix $g_{\mathrm{eff}}$ is an effective matrix for the families $A(t, \theta ; g)$ and $\widehat{A}(t, \boldsymbol{\theta} ; g)$ simultaneously.

In particular, the main effective matrix $g^{0}$ corresponding to the family $\widehat{A}(t, \theta ; g)$ is also an effective matrix for the family $A(t, \theta ; g)=f^{*} \widehat{A}(t, \theta ; g) f$. It is natural to associate the operator

$$
\mathcal{A}=f^{*} \widehat{\mathcal{A}} f
$$

with the operator

$$
\mathcal{A}^{0}=f^{*} \widehat{\mathcal{A}}^{0} f
$$

acting in $L_{2}\left(\mathbb{R}^{d} ; \mathbb{C}^{n}\right)$. Here $\widehat{\mathcal{A}}^{0}$ is defined by (1.10). Recall that $\widehat{\mathcal{A}}^{0}$ will not change if in (1.10) we replace $g^{0}$ by any other effective matrix $g_{\text {eff }}$. Consequently, the operator $\mathcal{A}^{0}$ also does not depend on the choice of the matrix $g_{\text {eff }}$. Thus, the operator (1.29) is defined via the operator (1.28) uniquely. We agree to call the operator $\mathcal{A}^{0}$ the effective DO for the $D O \mathcal{A}$.

We distinguish the case where $n=1$. Then $\widehat{S}(\theta ; g)$ (see (1.9)) is the operator of multiplication by the number $\widehat{\gamma}(\boldsymbol{\theta})=b(\boldsymbol{\theta})^{*} g^{0} b(\boldsymbol{\theta})$. By (1.1.26) and (1.27), the operator $S(\theta ; g)$ is reduced to multiplication by the number

$$
\gamma(\boldsymbol{\theta})=\widehat{\gamma}(\boldsymbol{\theta})|\Omega|\left\|f^{-1}\right\|_{L_{2}(\Omega)}^{-2}, \quad \widehat{\gamma}(\boldsymbol{\theta})=b(\boldsymbol{\theta})^{*} g^{0} b(\boldsymbol{\theta}) .
$$

\section{$\S 2$. Behavior of the Resolvent $\left(\mathcal{A}+\varepsilon^{2} I\right)^{-1}$ AS $\varepsilon \rightarrow 0$}

As in $\S 1$, we start with the case where $f=\mathbf{1}_{n}$, and then pass to the general case. 
2.1. The resolvent of the operator $\widehat{\mathcal{A}}$. We consider the resolvent $\left(\widehat{\mathcal{A}}+\varepsilon^{2} I\right)^{-1}$ of the operator $\widehat{\mathcal{A}}=b(\mathbf{D})^{*} g(\mathbf{x}) b(\mathbf{D})$ acting in $L_{2}\left(\mathbb{R}^{d} ; \mathbb{C}^{n}\right)$. We rely on Theorem 1.5.6, which is applied to the families $\widehat{\mathcal{A}}(t, \boldsymbol{\theta} ; g)$ and $\widehat{\mathcal{A}}\left(t, \boldsymbol{\theta} ; g^{0}\right)$ of the form (2.3.4). Here $g^{0}$ is the main effective matrix for $g(\mathbf{x})$.

Recall that $\alpha_{0}$ and $\alpha_{1}$ are the constants occurring in (2.1.3), and $r_{0}$ is the radius of the ball inscribed into the Brillouin zone $\left(r_{0}\right.$ is defined by (2.1.14)). In the notation of the constants we use the mark " $\wedge$ " to distinguish the case of $f=\mathbf{1}_{n}$. In accordance with $(2.2 .11)$,

$$
\widehat{c}_{*}=\alpha_{0}\left\|g^{-1}\right\|_{L_{\infty}}^{-1} .
$$

Recalling (2.3.7), we fix

$$
\widehat{\delta}=\left(r_{0} / 2\right)^{2} \alpha_{0}\left\|g^{-1}\right\|_{L_{\infty}}^{-1} .
$$

Next, in accordance with (2.3.8) and (2.2),

$$
\widehat{t}^{0}=\widehat{t}^{0}(\widehat{\delta})=\left(r_{0} / 2\right)\left(\alpha_{0} \alpha_{1}^{-1}\right)^{1 / 2}\left(\left\|g^{-1}\right\|_{L_{\infty}}\|g\|_{L_{\infty}}\right)^{-1 / 2} .
$$

Since $\widehat{\delta}$ is fixed, we do not need to indicate the dependence of $\widehat{t}^{0}$ on $\widehat{\delta}$ anymore.

Now, we must specify the constant $\mathcal{C}$ in (1.5.12). Using (1.5.10), (1.5.9), (1.5.6), (1.5.7), and (2.1) subsequently, we find

$$
\widehat{\mathcal{C}}=\alpha_{0}^{-1 / 2}\left\|g^{-1}\right\|_{L_{\infty}}^{1 / 2}\left(2 \beta_{1}\left(\widehat{t}^{0}\right)^{-1}+\beta_{2} \alpha_{0}^{-1}\left\|g^{-1}\right\|_{L_{\infty}} \widehat{\delta}\left(\widehat{t}^{0}\right)^{-3}\right) .
$$

Observe that, by (1.21), $\left\|g^{0}\right\|_{L_{\infty}} \leq\|g\|_{L_{\infty}}$ and $\left\|\left(g^{0}\right)^{-1}\right\|_{L_{\infty}} \leq\left\|g^{-1}\right\|_{L_{\infty}}$. It follows that if $g$ is replaced by $g^{0}$, then the quantities (2.1)-(2.3) may only become larger, and the constant (2.4) may only become smaller. Therefore, Theorem 1.5.6 leads directly to the following result. In its statement, we use the notation $(2.3 .1), \mathbf{k}=t \boldsymbol{\theta}$, and $\widehat{\mathcal{A}}(\mathbf{k} ; g)=\widehat{A}(t, \boldsymbol{\theta} ; g), \widehat{\mathcal{A}}\left(\mathbf{k} ; g^{0}\right)=\widehat{A}\left(t, \boldsymbol{\theta} ; g^{0}\right)$.

Proposition 2.1. For the operator

$$
\widehat{\mathcal{G}}(\varepsilon, \mathbf{k})=\left(\widehat{\mathcal{A}}(\mathbf{k} ; g)+\varepsilon^{2} I\right)^{-1}-\left(\widehat{\mathcal{A}}\left(\mathbf{k} ; g^{0}\right)+\varepsilon^{2} I\right)^{-1},
$$

we have

$$
\varepsilon\|\widehat{\mathcal{G}}(\varepsilon, \mathbf{k})\|_{\mathfrak{H} \rightarrow \mathfrak{H}} \leq \widehat{\mathcal{C}}+2 \varepsilon(3 \widehat{\delta})^{-1}, \quad \varepsilon>0, \quad|\mathbf{k}| \leq \widehat{t}^{0},
$$

where the constants $\widehat{\delta}, \widehat{t}^{0}$, and $\widehat{\mathcal{C}}$ are defined by (2.2)-(2.4).

Together with (2.6), we need estimates of resolvents for $\mathbf{k} \in \operatorname{clos} \widetilde{\Omega} \cap\left\{\mathbf{k}:|\mathbf{k}|>\widehat{t}^{0}\right\}$. By (2.2.13),

$$
\left\|\left(\widehat{\mathcal{A}}(\mathbf{k} ; g)+\varepsilon^{2} I\right)^{-1}\right\|_{\mathfrak{H} \rightarrow \mathfrak{H}} \leq \widehat{c}_{*}-1\left(\widehat{t}^{0}\right)^{-2}, \quad \mathbf{k} \in \operatorname{clos} \widetilde{\Omega} \cap\left\{\mathbf{k}:|\mathbf{k}|>\widehat{t}^{0}\right\},
$$

and the same estimate remains true with $g$ replaced by $g^{0}$. Therefore, we have the following statement.

Proposition 2.2. The following estimates are valid, where the notation (2.1)-(2.5) is used:

$$
\begin{aligned}
\varepsilon\|\widehat{\mathcal{G}}(\varepsilon, \mathbf{k})\|_{\mathfrak{H} \rightarrow \mathfrak{H}} & \leq \widehat{\mathcal{C}}_{\times}, \quad 0<\varepsilon \leq 1, \quad \mathbf{k} \in \operatorname{clos} \widetilde{\Omega}, \\
\widehat{\mathcal{C}}_{\times} & =\max \left\{\widehat{\mathcal{C}}+2(3 \widehat{\delta})^{-1}, 2 \widehat{\mathcal{C}}_{*}-1\left(\widehat{t}^{0}\right)^{-2}\right\} ; \\
& \limsup _{\varepsilon \rightarrow 0} \varepsilon\|\widehat{\mathcal{G}}(\varepsilon, \mathbf{k})\|_{\mathfrak{H} \rightarrow \mathfrak{H}} \leq \widehat{\mathcal{C}} .
\end{aligned}
$$


Now, it is easy to obtain one of the main results of the paper. We estimate the difference between the resolvents of the operator $\widehat{\mathcal{A}}=\widehat{\mathcal{A}}(g)$ and of the effective DO $\widehat{\mathcal{A}}^{0}=\widehat{\mathcal{A}}\left(g^{0}\right)$. We use the representation $(2.2 .17)$ for $\widehat{\mathcal{A}}(g)$ viewed as an operator acting in the direct integral $\mathcal{H}$ (see $(2.1 .18)$ ). Now, the required relations can be written as

$$
\mathcal{H}=\int_{\widetilde{\Omega}} \oplus \mathfrak{H} d \mathbf{k}, \quad \widehat{\mathcal{A}}(g)=\mathcal{U}^{-1}\left(\int_{\widetilde{\Omega}} \oplus \widehat{\mathcal{A}}(\mathbf{k} ; g) d \mathbf{k}\right) \mathcal{U},
$$

where $\mathcal{U}$ is a unitary mapping of the space $\mathfrak{G}:=L_{2}\left(\mathbb{R}^{d} ; \mathbb{C}^{n}\right)$ onto $\mathcal{H}$. From $(2.10)$ it follows that

$$
\left(\widehat{\mathcal{A}}(g)+\varepsilon^{2} I\right)^{-1}=\mathcal{U}^{-1}\left(\int_{\widetilde{\Omega}} \oplus\left(\widehat{\mathcal{A}}(\mathbf{k} ; g)+\varepsilon^{2} I\right)^{-1} d \mathbf{k}\right) \mathcal{U}
$$

A similar representation is valid with $g$ replaced by $g^{0}$, and also for the difference of the resolvents. Combining this with estimates (2.8) and (2.9), we arrive at the following theorem.

Theorem 2.3. Let $\widehat{\mathcal{A}}(g)=b(\mathbf{D})^{*} g(\mathbf{x}) b(\mathbf{D})$, and let $\widehat{\mathcal{A}}^{0}=\widehat{\mathcal{A}}\left(g^{0}\right)=b(\mathbf{D})^{*} g^{0} b(\mathbf{D})$ be the effective $D O$ for the $D O \widehat{\mathcal{A}}(g)$. Then

$$
\begin{aligned}
\varepsilon\left\|\left(\widehat{\mathcal{A}}(g)+\varepsilon^{2} I\right)^{-1}-\left(\widehat{\mathcal{A}}\left(g^{0}\right)+\varepsilon^{2} I\right)^{-1}\right\|_{\mathfrak{G} \rightarrow \mathfrak{G}} \leq \widehat{\mathcal{C}} \times, \quad 0<\varepsilon \leq 1, \\
\limsup _{\varepsilon \rightarrow 0} \varepsilon\left\|\left(\widehat{\mathcal{A}}(g)+\varepsilon^{2} I\right)^{-1}-\left(\widehat{\mathcal{A}}\left(g^{0}\right)+\varepsilon^{2} I\right)^{-1}\right\|_{\mathfrak{G} \rightarrow \mathfrak{G}} \leq \widehat{\mathcal{C}},
\end{aligned}
$$

where the constants $\widehat{\mathcal{C}}_{\times}$and $\widehat{\mathcal{C}}$ are the same as in (2.8) and (2.9).

Estimates (2.11) and (2.12) show that the norm of the difference of the resolvents is of order $O\left(\varepsilon^{-1}\right)$. At the same time, the norm of each resolvent is $O\left(\varepsilon^{-2}\right)$. The reason for this compensation in the difference is that the germs of the families $\widehat{\mathcal{A}}(\mathbf{k} ; g)$ and $\widehat{\mathcal{A}}\left(\mathbf{k} ; g^{0}\right)$ coincide for $\mathbf{k}=0$. Thus, in Theorem 2.3, the threshold effect near the edge of the spectrum is taken into account.

Remark 2.4. We emphasize once again that, in fact, the effective operator $\widehat{\mathcal{A}}^{0}=\widehat{\mathcal{A}}\left(g^{0}\right)$ does not depend on the choice of the effective matrix, but is determined by the initial operator $\widehat{\mathcal{A}}=\widehat{\mathcal{A}}(g)$. In general, the latter operator may also be described by a nonunique $\Gamma$-periodic matrix $g(\mathbf{x})$.

2.2. The resolvent of the operator $\mathcal{A}$. Now, we lift the assumption $f=\mathbf{1}_{n}$ and consider the operator

$$
\mathcal{A}=f^{*} \widehat{\mathcal{A}} f=f^{*} b(\mathbf{D})^{*} g b(\mathbf{D}) f .
$$

The constants (2.1)-(2.4) should be changed in order to take the dependence on $f$ into account. In accordance with (2.2.11), (2.3.7), and (2.3.8), we put

$$
\begin{gathered}
c_{*}=\widehat{c}_{*}\left\|f^{-1}\right\|_{L_{\infty}}^{-2}=\alpha_{0}\left\|f^{-1}\right\|_{L_{\infty}}^{-2}\left\|g^{-1}\right\|_{L_{\infty}}^{-1}, \\
\delta=c_{*}\left(r_{0} / 2\right)^{2}=\widehat{\delta}\left\|f^{-1}\right\|_{L_{\infty}}^{-2}, \\
t^{0}=t^{0}(\delta)=\widehat{t}^{0}(\widehat{\delta})\left(\|f\|_{L_{\infty}}\left\|f^{-1}\right\|_{L_{\infty}}\right)^{-1} .
\end{gathered}
$$

Now, the role of the constant (2.4) is played by the constant

$$
\mathcal{C}=c_{*}^{-1 / 2}\left(2 \beta_{1}\left(t^{0}(\delta)\right)^{-1}+c_{*}^{-1} \beta_{2} \delta\left(t^{0}(\delta)\right)^{-3}\right) .
$$

Using the notation of Proposition 2.1, we put

$$
\mathcal{A}(\mathbf{k} ; g, f)=f^{*} \widehat{\mathcal{A}}(\mathbf{k} ; g) f, \quad \mathcal{A}\left(\mathbf{k} ; g^{0}, f\right)=f^{*} \widehat{\mathcal{A}}\left(\mathbf{k} ; g^{0}\right) f .
$$

Theorem 1.5.6 implies the following generalization of Proposition 2.1. 
Proposition 2.5. For the operator

$$
\mathcal{G}(\varepsilon, \mathbf{k})=\left(\mathcal{A}(\mathbf{k} ; g, f)+\varepsilon^{2} I\right)^{-1}-\left(\mathcal{A}\left(\mathbf{k} ; g^{0}, f\right)+\varepsilon^{2} I\right)^{-1},
$$

we have

$$
\varepsilon\|\mathcal{G}(\varepsilon, \mathbf{k})\|_{\mathfrak{H} \rightarrow \mathfrak{H}} \leq \mathcal{C}+2 \varepsilon(3 \delta)^{-1}, \quad \varepsilon>0, \quad|\mathbf{k}| \leq t^{0},
$$

where the constants $\delta, t^{0}$, and $\mathcal{C}$ are defined by $(2.15)-(2.17)$.

For $\mathcal{A}(\mathbf{k} ; g, f)$ and $\mathcal{A}\left(\mathbf{k} ; g^{0}, f\right)$, estimate $(2.7)$ remains true with $\widehat{c}_{*}$ replaced by $c_{*}$ and $\widehat{t}^{0}$ replaced by $t^{0}$. Combining this with (2.18), we obtain a generalization of Proposition 2.2 .

Proposition 2.6. The following estimates are valid, where we use the notation (2.14)(2.17):

$$
\begin{aligned}
\varepsilon\|\mathcal{G}(\varepsilon, \mathbf{k})\|_{\mathfrak{H} \rightarrow \mathfrak{H}} \leq \mathcal{C}_{\times}, \quad 0<\varepsilon \leq 1, \quad \mathbf{k} \in \operatorname{clos} \widetilde{\Omega}, \\
\mathcal{C}_{\times}=\max \left\{\mathcal{C}+2(3 \delta)^{-1}, 2 c_{*}^{-1}\left(t^{0}\right)^{-2}\right\} ; \\
\limsup _{\varepsilon \rightarrow 0} \varepsilon\|\mathcal{G}(\varepsilon, \mathbf{k})\|_{\mathfrak{H} \rightarrow \mathfrak{H}} \leq \mathcal{C} .
\end{aligned}
$$

Finally, we obtain the following theorem.

Theorem 2.7. Let $\mathcal{A}=\mathcal{A}(g, f)$ be the operator (2.13), and let $\mathcal{A}^{0}=\mathcal{A}\left(g^{0}, f\right)$ be the corresponding effective $D O$. Then

$$
\begin{aligned}
& \varepsilon\left\|\left(\mathcal{A}(g, f)+\varepsilon^{2} I\right)^{-1}-\left(\mathcal{A}\left(g^{0}, f\right)+\varepsilon^{2} I\right)^{-1}\right\|_{\mathfrak{G} \rightarrow \mathfrak{G}} \leq \mathcal{C}_{\times}, \quad 0<\varepsilon \leq 1, \\
& \limsup _{\varepsilon \rightarrow 0} \varepsilon\left\|\left(\mathcal{A}(g, f)+\varepsilon^{2} I\right)^{-1}-\left(\mathcal{A}\left(g^{0}, f\right)+\varepsilon^{2} I\right)^{-1}\right\|_{\mathfrak{G} \rightarrow \mathfrak{G}} \leq \mathcal{C},
\end{aligned}
$$

where the constants $\mathcal{C}_{\times}$and $\mathcal{C}$ are the same as in (2.19), (2.20).

Proof. This theorem can be deduced from Proposition 2.6 in the same way as Theorem 2.3 was deduced from Proposition 2.2.

In (2.21) and (2.22), the difference of the resolvents corresponding to $g$ and $g^{0}$ is estimated. The matrix $f$ is fixed. It is impossible to find a good approximation for the resolvent of $\mathcal{A}(g, f)$ with variable $f$ by the resolvent of some operator with constant coefficients. 2 A more convenient approximation than that in (2.21) and (2.22) can be found, but the approximating operator will not be a resolvent anymore. This will be done in the next section; see Theorem 3.4.

\section{§3. Behavior of the Generalized Resolvent AS $\varepsilon \rightarrow 0$}

3.1. We are going to establish an analog of Theorem 2.3 for the generalized resolvent $\left(\widehat{\mathcal{A}}(g)+\varepsilon^{2} Q\right)^{-1}$. Here $Q=Q(\mathbf{x})>0$ is a $\Gamma$-periodic $(n \times n)$-matrix-valued function such that $Q+Q^{-1} \in L_{\infty}$. We use the abstract results of Subsections 1.1.5 and 1.5.3, assuming that $\widehat{\mathfrak{H}}=\mathfrak{H}=L_{2}\left(\Omega ; \mathbb{C}^{n}\right)$. Also, we recall (see (1.1)) that

$$
\widehat{\mathfrak{N}}=\operatorname{Ker} \widehat{\mathcal{A}}(0 ; g)=\left\{\mathbf{u} \in \mathfrak{H}: \mathbf{u}=\mathbf{c} \in \mathbb{C}^{n}\right\} .
$$

Keeping in mind Theorem 1.5.8, we represent $Q$ as

$$
Q(\mathbf{x})=\left(f(\mathbf{x}) f(\mathbf{x})^{*}\right)^{-1} .
$$

By (1.5.14), now multiplication by the $\Gamma$-periodic matrix-valued function $f$ plays the role of the isomorphism $M$. From (3.1) we see directly that the block of the operator of multiplication by $Q$ in the subspace $\widehat{\mathfrak{N}}$ is multiplication by the constant matrix $\bar{Q}$, i.e., by the mean value of $Q(\mathbf{x})$ over $\Omega$. If we view the operator of multiplication by $\bar{Q}$ as an

\footnotetext{
${ }^{2}$ In this case, the name "effective DO" for $\mathcal{A}\left(g^{0}, f\right)$ with variable $f$ does not seem quite adequate.
} 
operator in the entire space $\mathfrak{H}$, then its block in $\widehat{\mathfrak{N}}$ also coincides with multiplication by $\bar{Q}$. We apply Theorem 1.5.8 in order to estimate the difference

$$
\mathcal{F}(\varepsilon, \mathbf{k})=\left(\widehat{\mathcal{A}}(\mathbf{k} ; g)+\varepsilon^{2} Q\right)^{-1}-\left(\widehat{\mathcal{A}}\left(\mathbf{k} ; g^{0}\right)+\varepsilon^{2} \bar{Q}\right)^{-1} .
$$

In accordance with Subsection 1.5.3, the constant $C=2^{-1} \mathcal{C}+(3 \delta)^{-1}$ in $(1.5 .20)-(1.5 .22)$ is expressed in terms of constants $(2.14)-(2.17)$ corresponding to the operator $\mathcal{A}(g, f)$, rather than to the operator $\widehat{\mathcal{A}}(g)$. Here $f$ is as in (3.2). We note that $\|\bar{Q}\| \leq\|Q\|_{L_{\infty}}$, $\left\|(\bar{Q})^{-1}\right\| \leq\left\|Q^{-1}\right\|_{L_{\infty}}$, and we put $f_{+}=(\bar{Q})^{-1 / 2}$. Then it is easily seen that the same constants (2.14)-(2.17) are also suitable for the operator $\mathcal{A}\left(g^{0}, f_{+}\right)$. Consequently, now in (1.5.22) we can take $C_{+}=C$ and replace $\left\|(\bar{Q})^{-1}\right\|$ by $\left\|Q^{-1}\right\|_{L_{\infty}}$. As a result, we obtain an analog of estimate (2.6). Namely, the following statement is true.

Proposition 3.1. For the operator $\mathcal{F}(\varepsilon, \mathbf{k})$ defined by $(3.3)$, we have

$$
\varepsilon\|\mathcal{F}(\varepsilon, \mathbf{k})\|_{\mathfrak{H} \rightarrow \mathfrak{H}} \leq\left(\mathcal{C}+2(3 \delta)^{-1}\right)\left\|Q^{-1}\right\|_{L_{\infty}}, \quad 0<\varepsilon \leq 1, \quad|\mathbf{k}| \leq t^{0}
$$

where the constants $\delta, t^{0}$, and $\mathcal{C}$ are defined by $(2.15)-(2.17)$.

As has already been mentioned, the operator $\mathcal{A}(\mathbf{k} ; g, f)$ satisfies $(2.7)$ with $\widehat{c}_{*}$ replaced by $c_{*}$ and $\widehat{t}^{0}$ replaced by $t^{0}$. Therefore, using (1.5.13), we obtain the estimate

$$
\left\|\left(\widehat{\mathcal{A}}(\mathbf{k} ; g)+\varepsilon^{2} Q\right)^{-1}\right\|_{\mathfrak{H} \rightarrow \mathfrak{H}} \leq\left\|Q^{-1}\right\|_{L_{\infty}} c_{*}^{-1}\left(t^{0}\right)^{-2}, \quad \mathbf{k} \in \operatorname{clos} \widetilde{\Omega} \cap\left\{\mathbf{k}:|\mathbf{k}|>t^{0}\right\} .
$$

The same estimate is valid for $\left(\widehat{\mathcal{A}}\left(\mathbf{k} ; g^{0}\right)+\varepsilon^{2} \bar{Q}\right)^{-1}$. Combining these estimates with Proposition 3.1, we arrive at the following.

Proposition 3.2. For the operator $\mathcal{F}(\varepsilon, \mathbf{k})$ defined by $(3.3)$, we have

$$
\varepsilon\|\mathcal{F}(\varepsilon, \mathbf{k})\|_{\mathfrak{H} \rightarrow \mathfrak{H}} \leq \mathcal{C}_{\times}\left\|Q^{-1}\right\|_{L_{\infty}}, \quad \mathbf{k} \in \operatorname{clos} \widetilde{\Omega}, \quad 0<\varepsilon \leq 1
$$

where $\mathcal{C}_{\times}$is the constant occurring in (2.19).

Finally, (3.4) implies the following theorem (cf. the deduction of Theorem 2.3 from Proposition 2.2).

Theorem 3.3. We have

$$
\varepsilon\left\|\left(\widehat{\mathcal{A}}(g)+\varepsilon^{2} Q\right)^{-1}-\left(\widehat{\mathcal{A}}\left(g^{0}\right)+\varepsilon^{2} \bar{Q}\right)^{-1}\right\|_{\mathfrak{G} \rightarrow \mathfrak{G}} \leq \mathcal{C}_{\times}\left\|Q^{-1}\right\|_{L_{\infty}}, \quad 0<\varepsilon \leq 1 .
$$

If $Q=I$, estimate (3.5) turns into (2.11).

3.2. Another approximation for the operator

$$
\left(\mathcal{A}(g, f)+\varepsilon^{2} I\right)^{-1},
$$

more practical than (2.21), easily follows from Theorem 3.3. Now we assume that the matrix-valued function $f$ is given, while $Q$ is defined by (3.2). Clearly,

$$
\left(\mathcal{A}(g, f)+\varepsilon^{2} I\right)^{-1}=f^{-1}\left(\widehat{\mathcal{A}}(g)+\varepsilon^{2} Q\right)^{-1}\left(f^{*}\right)^{-1} .
$$

We put

$$
\mathcal{F}(\varepsilon):=\left(\mathcal{A}(g, f)+\varepsilon^{2} I\right)^{-1}-f^{-1}\left(\widehat{\mathcal{A}}\left(g^{0}\right)+\varepsilon^{2} \bar{Q}\right)^{-1}\left(f^{*}\right)^{-1} .
$$

Then (3.7) implies that

$$
\mathcal{F}(\varepsilon)=f^{-1}\left(\left(\widehat{\mathcal{A}}(g)+\varepsilon^{2} Q\right)^{-1}-\left(\widehat{\mathcal{A}}\left(g^{0}\right)+\varepsilon^{2} \bar{Q}\right)^{-1}\right)\left(f^{*}\right)^{-1} .
$$

The latter expression is estimated with the help of (3.5). As a result, we obtain the following statement. 
Theorem 3.4. For the operator $\mathcal{F}(\varepsilon)$ defined by (3.8), we have

$$
\varepsilon\|\mathcal{F}(\varepsilon)\|_{\mathfrak{G} \rightarrow \mathfrak{G}} \leq \mathcal{C}_{\times}\left\|Q^{-1}\right\|_{L_{\infty}}\|Q\|_{L_{\infty}}, \quad 0<\varepsilon \leq 1,
$$

where the matrix-valued function $Q$ is defined by (3.2).

Remark 3.5. In (3.8) and (3.9), the operator (3.6) is approximated by the operator $f(\mathbf{x})^{-1}\left(\widehat{\mathcal{A}}\left(g^{0}\right)+\varepsilon^{2} \bar{Q}\right)^{-1}\left(f(\mathbf{x})^{*}\right)^{-1}$. Although this operator involves $\mathbf{x}$-dependent factors "from both sides", the generalized resolvent must be calculated for a DO with constant coefficients. That is why estimate (3.9) has a big advantage as compared to (2.21).

3.3. A generalization of Theorem 3.4. A result that is more self-contained can be obtained for the operator $\mathcal{A}(g, f)$ if we consider the generalized resolvent

$$
\left(\mathcal{A}(g, f)+\varepsilon^{2} \mathfrak{Q}\right)^{-1}
$$

instead of the resolvent (3.6). Here $\mathfrak{Q}(\mathbf{x})>0$ is a $\Gamma$-periodic $(n \times n)$-matrix-valued function such that $\mathfrak{Q}+\mathfrak{Q}^{-1} \in L_{\infty}$. We represent $\mathfrak{Q}(\mathbf{x})$ in the form (3.2),

$$
\mathfrak{Q}=\left(\varphi \varphi^{*}\right)^{-1},
$$

and put

$$
\mathfrak{f}=f \varphi, \quad Q=\left(\mathfrak{f f}^{*}\right)^{-1} .
$$

The following theorem can be deduced from Theorem 3.3 by the method used to derive Theorem 3.4.

Theorem 3.6. We have

$$
\begin{gathered}
\varepsilon\left\|\left(\mathcal{A}(g, f)+\varepsilon^{2} \mathfrak{Q}\right)^{-1}-f^{-1}\left(\widehat{\mathcal{A}}\left(g^{0}\right)+\varepsilon^{2} \bar{Q}\right)^{-1}\left(f^{*}\right)^{-1}\right\|_{\mathfrak{G} \rightarrow \mathfrak{G}} \\
\leq \mathcal{C}_{\times}(\mathfrak{f})\left\|f^{-1}\right\|_{L_{\infty}}^{2}\|f\|_{L_{\infty}}^{2}\left\|\mathfrak{Q}^{-1}\right\|_{L_{\infty}}, \quad 0<\varepsilon \leq 1,
\end{gathered}
$$

where $\mathcal{C}_{\times}(\mathfrak{f})$ is the constant $(2.19)$ recalculated after the replacement of $f$ by $\mathfrak{f}$ in (2.14)(2.17).

\section{Chapter 4. Homogenization Problems For PeRiodic DO'S}

We proceed to the study of homogenization problems, treating them as threshold effects near the lower edge of the spectrum. The present chapter contains general homogenization results for periodic operators

$$
\mathcal{A}(g, f)=f(\mathbf{x})^{*} b(\mathbf{D})^{*} g(\mathbf{x}) b(\mathbf{D}) f(\mathbf{x})=f(\mathbf{x})^{*} \widehat{\mathcal{A}}(g) f(\mathbf{x})
$$

acting in $\mathfrak{G}=L_{2}\left(\mathbb{R}^{d} ; \mathbb{C}^{n}\right)$. Further, in Chapters $5-7$, we discuss applications of these results to specific periodic DO's of mathematical physics.

We use the following notation for Hilbert spaces:

$$
\begin{array}{r}
\mathfrak{H}=L_{2}\left(\Omega ; \mathbb{C}^{n}\right), \quad \mathfrak{H}_{*}=L_{2}\left(\Omega ; \mathbb{C}^{m}\right), \quad \mathfrak{G}=L_{2}\left(\mathbb{R}^{d} ; \mathbb{C}^{n}\right), \\
\mathfrak{G}_{*}=L_{2}\left(\mathbb{R}^{d} ; \mathbb{C}^{m}\right), \quad \mathfrak{G}^{s}=H^{s}\left(\mathbb{R}^{d} ; \mathbb{C}^{n}\right), \quad s \in \mathbb{R} .
\end{array}
$$

Also, we use the obvious notation like $\mathfrak{G}_{\text {loc }}, \mathfrak{G}_{* \text {,loc }}$, etc. The symbol $(\mathbf{F}, \mathbf{u})_{\mathfrak{G}}$ with $\mathbf{u} \in \mathfrak{G}^{1}$ and $\mathbf{F} \in \mathfrak{G}^{-1}$ stands for the value of the functional $\mathbf{F}$ on the element $\mathbf{u}$. The symbol $w$ indicates passage to the weak limit. If $\phi$ is a measurable $\Gamma$-periodic function, we denote $\phi^{\varepsilon}(\mathbf{x})=\phi\left(\varepsilon^{-1} \mathbf{x}\right)$. We also recall the notation (3.0.1), (3.0.2).

The following elementary proposition will often be used in weak limit procedures.

Proposition 0.1. $1^{\circ}$. Let $\Psi$ be a $\Gamma$-periodic function of class $L_{2, \mathrm{loc}}\left(\mathbb{R}^{d}\right)$. Then

$$
\Psi^{\varepsilon} \underset{\varepsilon \rightarrow 0}{\stackrel{w}{\longrightarrow}} \bar{\Psi} \quad \text { in } L_{2, \mathrm{loc}}\left(\mathbb{R}^{d}\right)
$$


$2^{\circ}$. Let $G \in L_{\infty}\left(\mathbb{R}^{d}\right)$ be a $\Gamma$-periodic function and let $\Phi \in L_{1}\left(\mathbb{R}^{d}\right)$. Then

$$
\lim _{\varepsilon \rightarrow 0} \int_{\mathbb{R}^{d}} G^{\varepsilon}(\mathbf{x}) \Phi(\mathbf{x}) d \mathbf{x}=\bar{G} \int_{\mathbb{R}^{d}} \Phi(\mathbf{x}) d \mathbf{x} .
$$

Relation (0.1) is the well-known mean value property (see, e.g., ZhKO]), which easily follows from the Riemann-Lebesgue lemma. Relation (0.2) is a direct consequence of $(0.1)$.

\section{§1. Statement of the PRoblem}

1.1. Concept of homogenization. For the operators $\mathcal{A}(g, f)$, we consider the family of $(\varepsilon \Gamma)$-periodic operators $\mathcal{A}_{\varepsilon}(g, f)=\mathcal{A}\left(g^{\varepsilon}, f^{\varepsilon}\right), \varepsilon>0$. The coefficients of the operator $\mathcal{A}_{\varepsilon}$ are rapidly oscillating as $\varepsilon \rightarrow 0$. Homogenization theory studies the behavior of the solutions $\mathbf{u}_{\varepsilon}$ of the equation

$$
\mathcal{A}_{\varepsilon}(g, f) \mathbf{u}_{\varepsilon}+\mathbf{u}_{\varepsilon}=\mathbf{F}
$$

as $\varepsilon \rightarrow 0$. In other words, we deal with the behavior of the resolvent $\left(\mathcal{A}_{\varepsilon}(g, f)+I\right)^{-1}$ as $\varepsilon \rightarrow 0$. The simplest case is that of $f=\mathbf{1}_{n}$, i.e., the case of the equation

$$
\widehat{\mathcal{A}}_{\varepsilon}(g) \mathbf{u}_{\varepsilon}+\mathbf{u}_{\varepsilon}=\mathbf{F} .
$$

It turns out that, as $\varepsilon \rightarrow 0$, the solution $\mathbf{u}_{\varepsilon}$ of (1.1) converges (in an appropriate sense) to the solution $\mathbf{u}_{0}$ of the equation

$$
\widehat{\mathcal{A}}\left(g^{0}\right) \mathbf{u}_{0}+\mathbf{u}_{0}=\mathbf{F} .
$$

Here $g^{0}$ is the (main) effective matrix for the operator $\widehat{\mathcal{A}}(g)$, and $\widehat{\mathcal{A}}\left(g^{0}\right)$ is the (effective) DO with constant coefficients.

Along with convergence of solutions, we are interested in convergence of what is called flows: $\mathbf{p}_{\varepsilon} \rightarrow \mathbf{p}_{0}$. The flows are defined by the formulas

$$
\mathbf{p}_{\varepsilon}=g^{\varepsilon} b(\mathbf{D}) \mathbf{u}_{\varepsilon}, \quad \mathbf{p}_{0}=g^{0} b(\mathbf{D}) \mathbf{u}_{0} .
$$

Note that the operator $\widehat{\mathcal{A}}\left(g^{0}\right)$ and, with it, the solution $\mathbf{u}_{0}$ of (1.2) do not depend on the choice of the effective matrix. At the same time, the flow $\mathbf{p}_{0}$ is defined in terms of the main effective matrix $g^{0}$.

The convergence of solutions and flows for equation (1.1) with rapidly oscillating coefficients to the solution and the flow for equation (1.2) with constant coefficients is commonly interpreted as homogenization of the medium. Usually, the medium described by the matrix $g^{0}$ is called the homogenized medium relative to the medium described by the periodic matrix $g^{\varepsilon}$. Herewith, it is convenient to fix the operator $b(\mathbf{D})$, because, as a rule, this operator is responsible for the type of the physical process, rather than for its parameters. Often, the effective DO $\widehat{\mathcal{A}}\left(g^{0}\right)$ is also called the homogenized DO.

For more general operators $\mathcal{A}(g, f)$ with variable $f$, we have not succeeded in finding an operator of the same class with constant $f$ and $g$ and such that its resolvent is the limit of the resolvents of the operators $\mathcal{A}_{\varepsilon}(g, f)$. We must approximate the family of resolvents by another (as simple as possible) operator family depending on $\varepsilon$. Uniqueness of approximation is lost, and homogenization of the medium can be talked about only with some reservations (cf. the footnote after Theorem 2.7 in Chapter 3). However, the weaker the convergence of solutions, the wider the choice of a "candidate" for approximation. All these features are well illustrated by the results of the present chapter. 
1.2. About the nature of results. In $\S 2$, we present approximation theorems for the family of resolvents

$$
\left(\mathcal{A}_{\varepsilon}(g, f)+I\right)^{-1}
$$

with respect to the operator norm in $\mathfrak{G}=L_{2}\left(\mathbb{R}^{d} ; \mathbb{C}^{n}\right)$. We also consider approximations for generalized resolvents. (After this extension of the question, the results on the existence of the (at least, weak) limit become self-contained (see Subsection 2.4).) For the norm of the difference, not only do we obtain an estimate of the right order $O(\varepsilon)$, but we also control the constants in estimates efficiently. Our considerations lean upon Theorems $3.2 .3,3.2 .7,3.3 .3,3.3 .4$, and 3.3.6, which automatically imply the results about homogenization. This allows us to view homogenization as a threshold phenomenon of spectral nature. The theorems in $\S 2$ are the most original results of this paper, as applied to homogenization theory. Part of these results were published by the authors in [BSu2].

In particular, the estimates with respect to the operator norm in $\mathfrak{G}$ are suitable for interpolation and allow us to estimate $\mathbf{u}_{\varepsilon}-\mathbf{u}_{0}$ in the Sobolev classes $H^{s}, 0<s<1$ (see Subsection 2.3). However, in $\S 2$ we do not discuss convergence of flows. The reason is that, in principle, convergence of flows cannot be "too good". Therefore, in $\S \S 3$ and 4 we discuss other versions of limit procedures, namely, weak ones, which is more traditional for homogenization theory. The proofs are also close to traditional in spirit, though there are some differences related to the fact that we consider a rather general class of operators. However, the material of $\S \S 3$ and 4 contains some new observations. In particular, we distinguish conditions under which the convergence of solutions or flows turns out to be strong.

It is convenient to postpone the further discussion till the comments at the end of this chapter.

\section{§2. Approximation of the Resolvent in the operator NORM}

\subsection{The case of the family $\widehat{\mathcal{A}}_{\varepsilon}(g)$. Here}

$$
\widehat{\mathcal{A}}_{\varepsilon}(g)=b(\mathbf{D})^{*} g^{\varepsilon}(\mathbf{x}) b(\mathbf{D}), \quad \varepsilon>0 .
$$

As before, $g(\mathbf{x})=h(\mathbf{x})^{*} h(\mathbf{x})$, and the $(m \times m)$-matrix $h(\mathbf{x})$ is invertible, $\Gamma$-periodic, and such that $h, h^{-1} \in L_{\infty}\left(\mathbb{R}^{d}\right)$. The selfadjoint operator (2.1) in $\mathfrak{G}$ is generated by the quadratic form

$$
\widehat{a}_{\varepsilon}(g)[\mathbf{u}, \mathbf{u}]=\left(g^{\varepsilon}(\mathbf{x}) b(\mathbf{D}) \mathbf{u}, b(\mathbf{D}) \mathbf{u}\right)_{\mathfrak{G}_{*}}, \quad \mathbf{u} \in \mathfrak{G}^{1} .
$$

Clearly, $\widehat{\mathcal{A}}_{1}(g)$ coincides with the operator $\widehat{\mathcal{A}}(g)$ that was discussed in Theorem 3.2.3.

We denote by $T_{\varepsilon}$ the following unitary scale transformation in $\mathfrak{G}:\left(T_{\varepsilon} \mathbf{u}\right)(\cdot)=\varepsilon^{d / 2} \mathbf{u}(\varepsilon \cdot)$. From (2.2) it follows directly that $\widehat{\mathcal{A}}_{\varepsilon}(g)=\varepsilon^{-2} T_{\varepsilon}^{*} \widehat{\mathcal{A}}(g) T_{\varepsilon}$, whence

$$
\left(\widehat{\mathcal{A}}_{\varepsilon}(g)+I\right)^{-1}=\varepsilon^{2} T_{\varepsilon}^{*}\left(\widehat{\mathcal{A}}(g)+\varepsilon^{2} I\right)^{-1} T_{\varepsilon} .
$$

If the matrix is constant, then (2.3) turns into an even simpler relation:

$$
\left(\widehat{\mathcal{A}}\left(g^{0}\right)+I\right)^{-1}=\varepsilon^{2} T_{\varepsilon}^{*}\left(\widehat{\mathcal{A}}\left(g^{0}\right)+\varepsilon^{2} I\right)^{-1} T_{\varepsilon} .
$$

Here $\widehat{\mathcal{A}}\left(g^{0}\right)=\widehat{\mathcal{A}}^{0}$ is the effective DO for the DO $\widehat{\mathcal{A}}(g)$. Subtracting (2.4) from (2.3) and applying Theorem 3.2.3, we obtain the following statement.

Theorem 2.1. Let $\widehat{\mathcal{A}}^{0}=\widehat{\mathcal{A}}\left(g^{0}\right)$ be the effective $D O$ for the $D O \widehat{\mathcal{A}}(g)=b(\mathbf{D})^{*} g(\mathbf{x}) b(\mathbf{D})$. Then

$$
\begin{gathered}
\left\|\left(\widehat{\mathcal{A}}_{\varepsilon}(g)+I\right)^{-1}-\left(\widehat{\mathcal{A}}^{0}+I\right)^{-1}\right\|_{\mathfrak{G} \rightarrow \mathfrak{G}} \leq \widehat{\mathcal{C}}_{\times} \varepsilon, \quad 0<\varepsilon \leq 1, \\
\limsup _{\varepsilon \rightarrow 0} \varepsilon^{-1}\left\|\left(\widehat{\mathcal{A}}_{\varepsilon}(g)+I\right)^{-1}-\left(\widehat{\mathcal{A}}^{0}+I\right)^{-1}\right\|_{\mathfrak{G} \rightarrow \mathfrak{G}} \leq \widehat{\mathcal{C}},
\end{gathered}
$$

where the constants $\widehat{\mathcal{C}}$ and $\widehat{\mathcal{C}}_{\times}$are defined by (3.2.4) and (3.2.8). 
Remark 2.2. The above scale transformation turns out to be efficient only because estimates in the operator norm have been obtained before. A direct way like this is unlikely to suit the study of other types of convergence.

2.2. Four more theorems follow equally easily from the results of Chapter 3 with the help of the scale transformation. We give the statements.

Theorem 2.3. Under the assumptions of Theorem 3.2.7, let $\mathcal{A}_{\varepsilon}(g, f)=\mathcal{A}\left(g^{\varepsilon}, f^{\varepsilon}\right)$ and $\mathcal{A}_{\varepsilon}\left(g^{0}, f\right)=\mathcal{A}\left(g^{0}, f^{\varepsilon}\right)$. Then

$$
\begin{gathered}
\left\|\left(\mathcal{A}_{\varepsilon}(g, f)+I\right)^{-1}-\left(\mathcal{A}_{\varepsilon}\left(g^{0}, f\right)+I\right)^{-1}\right\|_{\mathfrak{G} \rightarrow \mathfrak{G}} \leq \mathcal{C}_{\times} \varepsilon, \quad 0<\varepsilon \leq 1, \\
\limsup _{\varepsilon \rightarrow 0} \varepsilon^{-1}\left\|\left(\mathcal{A}_{\varepsilon}(g, f)+I\right)^{-1}-\left(\mathcal{A}_{\varepsilon}\left(g^{0}, f\right)+I\right)^{-1}\right\|_{\mathfrak{G} \rightarrow \mathfrak{G}} \leq \mathcal{C},
\end{gathered}
$$

where the constants $\mathcal{C}, \mathcal{C}_{\times}$are defined by (3.2.17) and (3.2.19).

In the following theorems we omit estimates for limsup. The first of these theorems carries estimate (2.5) over to the case of a generalized resolvent. Here the matrix-valued function $Q(\mathbf{x})$ is the same as in Subsection 3.3.1, and $f$ is defined in accordance with $(3.3 .2)$.

Theorem 2.4. We have

$$
\left\|\left(\widehat{\mathcal{A}}_{\varepsilon}(g)+Q^{\varepsilon}\right)^{-1}-\left(\widehat{\mathcal{A}}\left(g^{0}\right)+\bar{Q}\right)^{-1}\right\|_{\mathfrak{G} \rightarrow \mathfrak{G}} \leq \varepsilon \mathcal{C}_{\times}\left\|Q^{-1}\right\|_{L_{\infty}}, \quad 0<\varepsilon \leq 1,
$$

where $\mathcal{C}_{\times}$is the constant occurring in (3.2.19).

If $Q=I$, estimate (2.6) coincides with (2.5). In the following theorem, we give a more convenient approximation for the resolvent (1.4). Here $f$ and $Q$ are still related by $(3.3 .2)$.

Theorem 2.5. Under the above assumptions, we have

$$
\begin{aligned}
& \left\|\left(\mathcal{A}_{\varepsilon}(g, f)+I\right)^{-1}-\left(f^{\varepsilon}\right)^{-1}\left(\widehat{\mathcal{A}}\left(g^{0}\right)+\bar{Q}\right)^{-1}\left(\left(f^{\varepsilon}\right)^{*}\right)^{-1}\right\|_{\mathfrak{G} \rightarrow \mathfrak{G}} \\
& \quad \leq \varepsilon \mathcal{C}_{\times}\left\|Q^{-1}\right\|_{L_{\infty}}\|Q\|_{L_{\infty}}, \quad 0<\varepsilon \leq 1,
\end{aligned}
$$

where $\mathcal{C}_{\times}$is the constant occurring in (3.2.19).

Finally, we proceed to a statement that generalizes Theorem 2.5. Here we use the notation of Subsection 3.3.3.

Theorem 2.6. Under the assumptions and in the notation of Theorem 3.3.6, we have

$$
\begin{aligned}
& \left\|\left(\mathcal{A}_{\varepsilon}(g, f)+\mathfrak{Q}^{\varepsilon}\right)^{-1}-\left(f^{\varepsilon}\right)^{-1}\left(\widehat{\mathcal{A}}\left(g^{0}\right)+\bar{Q}\right)^{-1}\left(\left(f^{\varepsilon}\right)^{*}\right)^{-1}\right\|_{\mathfrak{G} \rightarrow \mathfrak{G}} \\
& \quad \leq \varepsilon \mathcal{C}_{\times}(\mathfrak{f})\left\|f^{-1}\right\|_{L_{\infty}}^{2}\|f\|_{L_{\infty}}^{2}\left\|\mathfrak{Q}^{-1}\right\|_{L_{\infty}}=: \varepsilon \mathcal{C}_{\nabla}, \quad 0<\varepsilon \leq 1 .
\end{aligned}
$$

These four theorems follow from Theorems 3.2.7, 3.3.3, 3.3.4, and 3.3.6 respectively, by the scale transformation. We omit the obvious arguments.

2.3. Interpolation. Along with (2.6), we have the following simple estimates for both generalized resolvents viewed as operators from $\mathfrak{G}^{-1}$ to $\mathfrak{G}^{1}$ :

$$
\left\|\left(\widehat{\mathcal{A}}_{\varepsilon}(g)+Q^{\varepsilon}\right)^{-1}\right\|_{\mathfrak{G}^{-1} \rightarrow \mathfrak{G}^{1}}+\left\|\left(\widehat{\mathcal{A}}\left(g^{0}\right)+\bar{Q}\right)^{-1}\right\|_{\mathfrak{G}^{-1} \rightarrow \mathfrak{G}^{1}} \leq 2 c_{+},
$$

where

$$
c_{+}^{-1}=\min \left\{\alpha_{0}\left\|g^{-1}\right\|_{L_{\infty}}^{-1},\left\|Q^{-1}\right\|_{L_{\infty}}^{-1}\right\} .
$$

The standard interpolation between (2.6) and (2.9) implies the following result. 
Theorem 2.7. Under the assumptions of Theorem 2.4, we have

$$
\begin{array}{r}
\left\|\left(\widehat{\mathcal{A}}_{\varepsilon}(g)+Q^{\varepsilon}\right)^{-1}-\left(\widehat{\mathcal{A}}\left(g^{0}\right)+\bar{Q}\right)^{-1}\right\|_{\mathfrak{G}^{-s} \rightarrow \mathfrak{G}^{s}} \leq \varepsilon^{1-s}\left(2 c_{+}\right)^{s}\left(\mathcal{C}_{\times}\left\|Q^{-1}\right\|_{L_{\infty}}\right)^{1-s} \\
0 \leq s<1, \quad 0<\varepsilon \leq 1 .
\end{array}
$$

If $Q=I$, the expressions for the constants in (2.10) and (2.11) simplify.

Under the assumptions of Theorems 2.3, 2.5, and 2.6, the interpolation is less natural, because we only have $f^{\varepsilon} \mathbf{u}_{\varepsilon} \in \mathfrak{G}^{1}$. However, it is still easy to interpolate the properties of the product $f^{\varepsilon} \mathbf{u}_{\varepsilon}$. We formulate the corresponding result, based on Theorem 2.5. We put

$$
\mathcal{W}(\varepsilon)=f^{\varepsilon}\left(\mathcal{A}_{\varepsilon}(g, f)+I\right)^{-1}-\left(\widehat{\mathcal{A}}\left(g^{0}\right)+\bar{Q}\right)^{-1}\left(\left(f^{\varepsilon}\right)^{*}\right)^{-1} .
$$

By (2.7), for the operator (2.12) we have

$$
\|\mathcal{W}(\varepsilon)\|_{\mathfrak{G} \rightarrow \mathfrak{G}} \leq \varepsilon \mathcal{C}_{\times}\left\|Q^{-1}\right\|_{L_{\infty}}^{3 / 2}\|Q\|_{L_{\infty}}=: \varepsilon \mathcal{C}_{\triangle}, \quad 0<\varepsilon \leq 1 .
$$

Moreover, it is easily seen that

$$
\left\|f^{\varepsilon}\left(\mathcal{A}_{\varepsilon}(g, f)+I\right)^{-1}\right\|_{\mathfrak{G} \rightarrow \mathfrak{G}^{1}}+\left\|\left(\widehat{\mathcal{A}}\left(g^{0}\right)+\bar{Q}\right)^{-1}\left(\left(f^{\varepsilon}\right)^{*}\right)^{-1}\right\|_{\mathfrak{G}^{\prime} \rightarrow \mathfrak{G}^{1}} \leq 2 c_{+}\left\|f^{-1}\right\|_{L_{\infty}}=: c_{\triangle} .
$$

Here $c_{+}$is defined by (2.10). Interpolating between (2.13) and (2.14), we arrive at the following statement.

Theorem 2.8. Under the assumptions of Theorem 2.5, we have

$$
\|\mathcal{W}(\varepsilon)\|_{\mathfrak{G} \rightarrow \mathfrak{G}^{s}} \leq \varepsilon^{1-s} c_{\triangle}^{s} \mathcal{C}_{\triangle}^{1-s}, \quad 0 \leq s<1, \quad 0<\varepsilon \leq 1 .
$$

2.4. On the weak limit for the generalized resolvent. Under the assumptions of Theorem 2.6, the weak operator limit in $\mathfrak{G}$ can be found for the generalized resolvent

$$
\left(\mathcal{A}_{\varepsilon}(g, f)+\mathfrak{Q}^{\varepsilon}\right)^{-1} .
$$

Indeed, by (2.8), it suffices to find the $(w)$-limit for the operator

$$
\mathcal{R}_{\varepsilon}(f)=\left(f^{\varepsilon}\right)^{-1}\left(\widehat{\mathcal{A}}\left(g^{0}\right)+\bar{Q}\right)^{-1}\left(\left(f^{\varepsilon}\right)^{*}\right)^{-1} .
$$

Since the $(\mathfrak{G} \rightarrow \mathfrak{G})$-norm of $\mathcal{R}_{\varepsilon}(f)$ is bounded with respect to $\varepsilon$, it suffices to find the limit

$$
\lim _{\varepsilon \rightarrow 0}\left(\mathcal{R}_{\varepsilon}(f) \mathbf{F}, \mathbf{G}\right)_{\mathfrak{G}}, \quad \mathbf{F}, \mathbf{G} \in C_{0}^{\infty}\left(\mathbb{R}^{d} ; \mathbb{C}^{n}\right)
$$

By the mean value property (see Proposition 0.1), we have

$$
(w, \mathfrak{G})-\lim _{\varepsilon \rightarrow 0}\left(\left(f^{\varepsilon}\right)^{*}\right)^{-1} \mathbf{F}=\left(\underline{f}^{*}\right)^{-1} \mathbf{F}, \quad(w, \mathfrak{G})-\lim _{\varepsilon \rightarrow 0}\left(\left(f^{\varepsilon}\right)^{*}\right)^{-1} \mathbf{G}=\left(\underline{f}^{*}\right)^{-1} \mathbf{G} .
$$

Let $\eta \in C_{0}^{\infty}\left(\mathbb{R}^{d}\right)$ be such that $\eta(\mathbf{x})=1$ for $\mathbf{x} \in \operatorname{supp} \mathbf{F}$. Then, in (2.16), the operator $\left(\widehat{\mathcal{A}}\left(g^{0}\right)+\bar{Q}\right)^{-1}$ can be replaced by the compact operator $\left(\widehat{\mathcal{A}}\left(g^{0}\right)+\bar{Q}\right)^{-1} \eta$. Now, it is clear that the limit in (2.16) is equal to

$$
\left(\left(\widehat{\mathcal{A}}\left(g^{0}\right)+\bar{Q}\right)^{-1}\left(\underline{f}^{*}\right)^{-1} \mathbf{F},\left(\underline{f}^{*}\right)^{-1} \mathbf{G}\right)_{\mathfrak{G}} .
$$

As a result, we obtain the following theorem.

Theorem 2.9. Under the assumptions of Theorem 2.6, the weak limit for the resolvent (2.15) exists:

$$
\begin{aligned}
(w, \mathfrak{G} & \rightarrow \mathfrak{G})-\lim _{\varepsilon \rightarrow 0}\left(\mathcal{A}_{\varepsilon}(g, f)+\mathfrak{Q}^{\varepsilon}\right)^{-1}=(\underline{f})^{-1}\left(\widehat{\mathcal{A}}\left(g^{0}\right)+\bar{Q}\right)^{-1}\left((\underline{f})^{-1}\right)^{*} \\
& =\left(\mathcal{A}\left(g^{0}, \underline{f}\right)+Q_{0}\right)^{-1},
\end{aligned}
$$

where the constant matrix $Q_{0}$ is given by the formula $Q_{0}=\underline{f}^{*} \bar{Q} \underline{f}=\underline{f}^{*}(\underline{\mathfrak{f f}})^{-1} \underline{f}$. 
The operator on the right-hand side of (2.17) is a generalized resolvent for $\mathcal{A}\left(g^{0}, f\right)$. However, the expression for $Q_{0}$ in terms of $f$ and $\mathfrak{Q}$ is fairly involved. On the other hand, the "homogenized" matrix $Q_{0}$ does not depend on $b(\mathbf{D})$ and $g$. Observe also that if $\mathfrak{Q}=\mathbf{1}_{n}$, then $Q_{0}=f^{*}\left(f f^{*}\right)^{-1} f \neq \mathbf{1}_{n}$, provided $f \neq$ const. The existence of the limit for the family (2.15) is related to the essential weakening of the "quality" of the limit procedure.

\section{§3. WeAK CONVERGENCE OF SOlutions AND FLOWS}

3.1. Under the assumptions of Theorem 2.7, the solutions $\mathbf{u}_{\varepsilon}$ of the equations

$$
\left(\widehat{\mathcal{A}}_{\varepsilon}(g)+Q^{\varepsilon}(\mathbf{x})\right) \mathbf{u}_{\varepsilon}=\mathbf{F}
$$

converge (with good control) in $\mathfrak{G}^{s}, s<1$, to the solution $\mathbf{u}_{0}$ of the homogenized equation

$$
\left(\widehat{\mathcal{A}}\left(g^{0}\right)+\bar{Q}\right) \mathbf{u}_{0}=\mathbf{F} .
$$

For $s=1$, a result of such a kind definitely fails, although the weak $\mathfrak{G}^{1}$-limit of the solutions exists, even for $\mathbf{F} \in \mathfrak{G}^{-1}$. Moreover, the weak $\mathfrak{G}$-limit of the flows exists. These results about convergence are traditional for homogenization theory. In this section, we prove a theorem of this kind for general equations of the form (3.1). Moreover, we assume that $\mathbf{F}$ depends on $\varepsilon$. This assumption will allow us to deduce a useful consequence (see Subsection 4.2).

Suppose $\mathbf{F}_{\varepsilon}, \mathbf{F}_{0} \in \mathfrak{G}^{-1}$, where the family $\left\{\mathbf{F}_{\varepsilon}\right\}$ is bounded in $\mathfrak{G}^{-1}$ :

$$
\left\|\mathbf{F}_{\varepsilon}\right\|_{\mathfrak{G}^{-1}} \leq C^{*} \text {. }
$$

Moreover, we assume that

$$
\left(\mathfrak{G}_{\text {loc }}^{-1}\right)-\lim _{\varepsilon \rightarrow 0} \mathbf{F}_{\varepsilon}=\mathbf{F}_{0} .
$$

Suppose that $Q(\mathbf{x})$ is a measurable $\Gamma$-periodic matrix-valued function such that $Q(\mathbf{x})>0$ and

$$
Q+Q^{-1} \in L_{\infty}
$$

Consider equations (3.1) and (3.2) with $\mathbf{F}=\mathbf{F}_{\varepsilon}$ and $\mathbf{F}=\mathbf{F}_{0}$, respectively. These equations are equivalent to the relations

$$
\begin{array}{rlrl}
\left(g^{\varepsilon} b(\mathbf{D}) \mathbf{u}_{\varepsilon}, b(\mathbf{D}) \mathbf{z}\right)_{\mathfrak{G}_{*}}+\left(Q^{\varepsilon} \mathbf{u}_{\varepsilon}, \mathbf{z}\right)_{\mathfrak{G}} & =\left(\mathbf{F}_{\varepsilon}, \mathbf{z}\right)_{\mathfrak{G}}, & & \mathbf{z} \in \mathfrak{G}^{1}, \\
\left(g^{0} b(\mathbf{D}) \mathbf{u}_{0}, b(\mathbf{D}) \mathbf{z}\right)_{\mathfrak{G}_{*}}+\left(\bar{Q} \mathbf{u}_{0}, \mathbf{z}\right)_{\mathfrak{G}}=\left(\mathbf{F}_{0}, \mathbf{z}\right)_{\mathfrak{G}}, & \mathbf{z} \in \mathfrak{G}^{1} .
\end{array}
$$

Let $c_{+}$be the constant occurring in (2.10). Combining (2.1.11) (for $f=\mathbf{1}_{n}$ ) with (3.3) and (3.6), we see that

$$
\left\|\mathbf{u}_{\varepsilon}\right\|_{\mathfrak{G}^{1}} \leq c_{+} C^{*}, \quad\left\|b(\mathbf{D}) u_{\varepsilon}\right\|_{\mathfrak{G}_{*}} \leq \alpha_{1}^{1 / 2} c_{+} C^{*},
$$

where $\alpha_{1}$ is the constant occurring in (2.1.3). Next, (3.8) implies that the flows (1.3) are bounded:

$$
\left\|\mathbf{p}_{\varepsilon}\right\|_{\mathfrak{G}_{*}} \leq \alpha_{1}^{1 / 2} c_{+} C^{*}\|g\|_{L_{\infty}} .
$$

Theorem 3.1. Suppose $\mathbf{u}_{\varepsilon}, \mathbf{u}_{0} \in \mathfrak{G}^{1}$ satisfy identities (3.6) and (3.7), respectively. Under the above assumptions about $Q, \mathbf{F}_{\varepsilon}$, and $\mathbf{F}_{0}$, the following statements are true:

$1^{\circ}$. As $\varepsilon \rightarrow 0$, the solutions $\mathbf{u}_{\varepsilon}$ converge to $\mathbf{u}_{0}$ weakly in $\mathfrak{G}^{1}$.

$2^{\circ}$. As $\varepsilon \rightarrow 0$, the flows $\mathbf{p}_{\varepsilon}$ converge to the flow $\mathbf{p}_{0}=g^{0} b(\mathbf{D}) \mathbf{u}_{0}$ weakly in $\mathfrak{G}_{*}$.

The remaining part of $\S 3$ is devoted to the proof of this theorem. 
3.2. Proof of Theorem 3.1. By (3.8) and (3.9), the limits

$$
\begin{gathered}
\left(w, \mathfrak{G}^{1}\right)-\lim _{\varepsilon_{j} \rightarrow 0} \mathbf{u}_{\varepsilon_{j}}=\mathbf{u} \in \mathfrak{G}^{1}, \\
\left(w, \mathfrak{G}_{*}\right)-\lim _{\varepsilon_{j} \rightarrow 0} \mathbf{p}_{\varepsilon_{j}}=\mathbf{p} \in \mathfrak{G}_{*}
\end{gathered}
$$

exist for some sequence $\varepsilon_{j} \rightarrow 0$. Our goal is to show that $\mathbf{u}=\mathbf{u}_{0}, \mathbf{p}=\mathbf{p}_{0}$. Then (3.10) and (3.11) will imply both conclusions of the theorem.

We repeat the representation (3.1.14) for $g^{0}$ :

$$
g^{0} \mathbf{C}=|\Omega|^{-1} \int_{\Omega} g(\mathbf{x})(b(\mathbf{D}) \mathbf{v}+\mathbf{C}) d \mathbf{x}, \quad \mathbf{C} \in \mathbb{C}^{m},
$$

where $\mathbf{v} \in \widetilde{H}^{1}\left(\Omega ; \mathbb{C}^{n}\right)$ is determined by (3.1.3) and by the condition $\overline{\mathbf{v}}=0$. We put $\boldsymbol{\psi}(\mathbf{x})=(b(\mathbf{D}) \mathbf{v})(\mathbf{x})+\mathbf{C}$ and extend $\boldsymbol{\psi}$ to a $\Gamma$-periodic function $\boldsymbol{\psi} \in \mathfrak{G}_{* \text {,loc. By Proposition }}$ $0.1\left(1^{\circ}\right)$, we have

$$
\left(w, \mathfrak{G}_{*, \operatorname{loc}}\right)-\lim _{\varepsilon \rightarrow 0} \boldsymbol{\psi}^{\varepsilon}=\mathbf{C},
$$

and, by (3.12),

$$
\left(w, \mathfrak{G}_{*, \text { loc }}\right)-\lim _{\varepsilon \rightarrow 0} g^{\varepsilon} \boldsymbol{\Psi}^{\varepsilon}=g^{0} \mathbf{C} .
$$

Now we need the following lemma.

Lemma 3.2. Let $\eta \in C_{0}^{\infty}\left(\mathbb{R}^{d}\right)$. Then

$$
\lim _{\varepsilon_{j} \rightarrow 0}\left(\eta \boldsymbol{\Psi}^{\varepsilon}, \mathbf{p}_{\varepsilon}\right)_{\mathfrak{G}_{*}}=(\eta \mathbf{C}, \mathbf{p})_{\mathfrak{G}_{*}} .
$$

Proof of Lemma 3.2. The expression under the limit sign splits into the sum

$$
\left(\eta \mathbf{C}, \mathbf{p}_{\varepsilon}\right)_{\mathfrak{G}_{*}}+\left(\eta(b(\mathbf{D}) \mathbf{v})^{\varepsilon}, \mathbf{p}_{\varepsilon}\right)_{\mathfrak{G}_{*}} .
$$

By (3.11), the limit of the first summand (as $\varepsilon_{j} \rightarrow 0$ ) coincides with the right-hand side of (3.14). Thus, the problem is reduced to checking that the second summand in (3.15) (we denote it by $\Theta(\varepsilon)$ ) tends to zero. We have

$$
\begin{aligned}
\Theta(\varepsilon) & =\varepsilon\left(\eta b(\mathbf{D}) \mathbf{v}^{\varepsilon}, \mathbf{p}_{\varepsilon}\right)_{\mathfrak{G}_{*}}=\varepsilon\left(b(\mathbf{D})\left(\eta \mathbf{v}^{\varepsilon}\right), g^{\varepsilon} b(\mathbf{D}) \mathbf{u}_{\varepsilon}\right)_{\mathfrak{G}_{*}}-\varepsilon\left(b(\mathbf{D} \eta) \mathbf{v}^{\varepsilon}, \mathbf{p}_{\varepsilon}\right)_{\mathfrak{G}_{*}} \\
& =: \varepsilon\left(\Theta_{1}(\varepsilon)-\Theta_{2}(\varepsilon)\right) .
\end{aligned}
$$

The matrix-valued function $b(\mathbf{D} \eta)$ has compact support (see the representation (2.1.4)). By (0.1) and the condition $\overline{\mathbf{v}}=0$, we have

$$
\left(w, \mathfrak{G}_{\text {loc }}\right)-\lim _{\varepsilon \rightarrow 0} \mathbf{v}^{\varepsilon}=0 .
$$

Combining this with (3.9), we see that $\lim _{\varepsilon \rightarrow 0} \varepsilon \Theta_{2}(\varepsilon)=0$.

It remains to show that

$$
\lim _{\varepsilon \rightarrow 0} \varepsilon \Theta_{1}(\varepsilon)=0
$$

Let $\mathcal{K} \subset \mathbb{R}^{d}$ be a ball satisfying $\mathcal{K} \supset \operatorname{supp} \eta$. It is easily seen that

$$
\left\|\eta \mathbf{v}^{\varepsilon}\right\|_{H^{1}\left(\mathcal{K} ; \mathbb{C}^{n}\right)} \leq C(\eta, \mathcal{K}) \varepsilon^{-1}\|\mathbf{v}\|_{H^{1}\left(\Omega ; \mathbb{C}^{n}\right)} .
$$

By (3.6) (with $\mathbf{z}=\eta \mathbf{v}^{\varepsilon}$ ),

$$
\Theta_{1}(\varepsilon)=\left(\eta \mathbf{v}^{\varepsilon}, \mathbf{F}_{\varepsilon}\right)_{\mathfrak{G}}-\left(\eta \mathbf{v}^{\varepsilon}, Q^{\varepsilon} \mathbf{u}_{\varepsilon}\right)_{\mathfrak{G}}=: \Theta_{11}(\varepsilon)-\Theta_{12}(\varepsilon) .
$$

By (3.4) and (3.18), we have $\lim _{\varepsilon \rightarrow 0} \varepsilon\left(\eta \mathbf{v}^{\varepsilon}, \mathbf{F}_{\varepsilon}-\mathbf{F}_{0}\right)_{\mathfrak{G}}=0$. Next, $\lim _{\varepsilon \rightarrow 0} \varepsilon\left(\eta \mathbf{v}^{\varepsilon}, \mathbf{F}_{0}\right)_{\mathfrak{G}}=0$. Indeed, by (3.18), we may assume that $\mathbf{F}_{0} \in \mathfrak{G}$, and then the required fact follows from (3.16). Thus, $\lim _{\varepsilon \rightarrow 0} \varepsilon \Theta_{11}(\varepsilon)=0$. Finally, relations (3.5), (3.8), and (3.16) show that $\lim _{\varepsilon \rightarrow 0} \varepsilon \Theta_{12}(\varepsilon)=0$. This yields (3.17). 
3.3. Proof of Theorem 3.1 (continuation). On the basis of the obvious identity

$$
\left\langle\boldsymbol{\psi}^{\varepsilon}, \mathbf{p}_{\varepsilon}\right\rangle_{\mathbb{C}^{m}}=\left\langle g^{\varepsilon} \boldsymbol{\psi}^{\varepsilon}, b(\mathbf{D}) \mathbf{u}_{\varepsilon}\right\rangle_{\mathbb{C}^{m}},
$$

one can calculate the limit in (3.14) by another method. Namely,

$$
\begin{aligned}
\left(\eta \boldsymbol{\psi}^{\varepsilon}, \mathbf{p}_{\varepsilon}\right)_{\mathfrak{G}_{*}} & =\left(\eta g^{\varepsilon} \boldsymbol{\psi}^{\varepsilon}, b(\mathbf{D}) \mathbf{u}_{\varepsilon}\right)_{\mathfrak{G}_{*}} \\
& =\left(\eta g^{\varepsilon} \boldsymbol{\psi}^{\varepsilon}, b(\mathbf{D}) \mathbf{u}\right)_{\mathfrak{G}_{*}}+\left(\eta g^{\varepsilon} \boldsymbol{\psi}^{\varepsilon}, b(\mathbf{D})\left(\mathbf{u}_{\varepsilon}-\mathbf{u}\right)\right)_{\mathfrak{G}_{*}} \\
& =: \Theta_{3}(\varepsilon)+\Theta_{4}(\varepsilon) .
\end{aligned}
$$

From (3.13) it follows that

$$
\lim _{\varepsilon \rightarrow 0} \Theta_{3}(\varepsilon)=\left(g^{0} \mathbf{C}, \eta^{+} b(\mathbf{D}) \mathbf{u}\right)_{\mathfrak{G}_{*}}=\left(\eta \mathbf{C}, g^{0} b(\mathbf{D}) \mathbf{u}\right)_{\mathfrak{G}_{*}} .
$$

Here $\eta^{+}$is the complex conjugate of $\eta$. We shall show that

$$
\lim _{\varepsilon_{j} \rightarrow 0} \Theta_{4}(\varepsilon)=0 .
$$

Relations (3.19)-(3.21) imply that

$$
\lim _{\varepsilon_{j} \rightarrow 0}\left(\eta \boldsymbol{\Psi}^{\varepsilon}, \mathbf{p}_{\varepsilon}\right)_{\mathfrak{G}_{*}}=\left(\eta \mathbf{C}, g^{0} b(\mathbf{D}) \mathbf{u}\right)_{\mathfrak{G}_{*}} .
$$

Comparing the right-hand sides of (3.14) and (3.22) for all $\eta \in C_{0}^{\infty}\left(\mathbb{R}^{d}\right), \mathbf{C} \in \mathbb{C}^{m}$, we obtain

$$
\mathbf{p}=g^{0} b(\mathbf{D}) \mathbf{u} .
$$

In order to prove that $\mathbf{u}=\mathbf{u}_{0}$ (and then $\mathbf{p}=\mathbf{p}_{0}$ ), we pass to the limit in (3.6) as $\varepsilon_{j} \rightarrow 0$. We may assume that $\mathbf{z} \in C_{0}^{\infty}\left(\mathbb{R}^{d} ; \mathbb{C}^{n}\right)$. Using (3.11) and (3.23), we obtain

$$
\left(g^{\varepsilon} b(\mathbf{D}) \mathbf{u}_{\varepsilon}, b(\mathbf{D}) \mathbf{z}\right)_{\mathfrak{G}_{*}}=\left(\mathbf{p}_{\varepsilon}, b(\mathbf{D}) \mathbf{z}\right)_{\mathfrak{G}_{*}} \underset{\varepsilon_{j} \rightarrow 0}{\longrightarrow}(\mathbf{p}, b(\mathbf{D}) \mathbf{z})_{\mathfrak{G}_{*}}=\left(g^{0} b(\mathbf{D}) \mathbf{u}, b(\mathbf{D}) \mathbf{z}\right)_{\mathfrak{G}_{*}} .
$$

Condition (3.4) implies that

$$
\left(\mathbf{F}_{\varepsilon}, \mathbf{z}\right)_{\mathfrak{G}} \underset{\varepsilon \rightarrow 0}{\longrightarrow}\left(\mathbf{F}_{0}, \mathbf{z}\right)_{\mathfrak{G}}, \quad \mathbf{z} \in C_{0}^{\infty}\left(\mathbb{R}^{d} ; \mathbb{C}^{n}\right) .
$$

Let $\mathcal{K}_{0}$ be a ball such that $\mathcal{K}_{0} \supset \operatorname{supp} \mathbf{z}$. From (3.10) we see that, as $\varepsilon_{j} \rightarrow 0$, the $\mathbf{u}_{\varepsilon_{j}}$ converge to $\mathbf{u}$ in $L_{2}\left(\mathcal{K}_{0} ; \mathbb{C}^{n}\right)$. Combining this with (0.1), we arrive at

$$
\left(Q^{\varepsilon} \mathbf{u}_{\varepsilon}, \mathbf{z}\right)_{\mathfrak{G}} \underset{\varepsilon_{j} \rightarrow 0}{\longrightarrow}(\bar{Q} \mathbf{u}, \mathbf{z})_{\mathfrak{G}}, \quad \mathbf{z} \in C_{0}^{\infty}\left(\mathbb{R}^{d} ; \mathbb{C}^{n}\right) .
$$

As a result, by (3.24)-(3.26), passage to the limit in (3.6) yields (3.7) with $\mathbf{u}_{0}$ replaced by $\mathbf{u}$. Thus, $\mathbf{u}=\mathbf{u}_{0}$ and $\mathbf{p}=\mathbf{p}_{0}$, which is equivalent to the conclusions of the theorem.

3.4. Proof of Theorem 3.1 (end). It remains to check (3.21). We represent $\Theta_{4}(\varepsilon)$ as

$$
\begin{aligned}
\Theta_{4}(\varepsilon) & =-\left(g^{\varepsilon} \boldsymbol{\psi}^{\varepsilon}, b\left(\mathbf{D} \eta^{+}\right)\left(\mathbf{u}_{\varepsilon}-\mathbf{u}\right)\right)_{\mathfrak{G}_{*}}+\left(g^{\varepsilon} \boldsymbol{\psi}^{\varepsilon}, b(\mathbf{D}) \eta^{+}\left(\mathbf{u}_{\varepsilon}-\mathbf{u}\right)\right)_{\mathfrak{G}_{*}} \\
& =: \Theta_{5}(\varepsilon)+\Theta_{6}(\varepsilon) .
\end{aligned}
$$

The matrix-valued function $b\left(\mathbf{D} \eta^{+}\right)$has compact support. From (3.13) and the convergence of $\mathbf{u}_{\varepsilon_{j}}$ to $\mathbf{u}$ in $L_{2}\left(\mathcal{K} ; \mathbb{C}^{n}\right)$ it follows that $\Theta_{5}\left(\varepsilon_{j}\right) \rightarrow 0$ as $\varepsilon_{j} \rightarrow 0$. Now we show that

$$
\Theta_{6}(\varepsilon)=0, \quad \varepsilon>0
$$

which implies (3.21). 
The scale transformation allows us to rewrite (3.27) as

$$
\int_{\widetilde{\mathcal{K}}}\langle g \boldsymbol{\Psi}, b(\mathbf{D}) \mathbf{z}\rangle_{\mathbb{C}^{m}} d \mathbf{x}=0,
$$

where $\widetilde{\mathcal{K}}$ is a ball, and $\mathbf{z}$ is a function such that $\mathbf{z} \in \mathfrak{G}^{1}$ and $\operatorname{supp} \mathbf{z} \subset \widetilde{\mathcal{K}}$. (Since $\varepsilon$ is fixed now, we do not reflect the dependence of $\widetilde{\mathcal{K}}$ and $\mathbf{z}$ on $\varepsilon$ in the notation.) We choose an appropriate "partition of unity" $\left\{\zeta_{l}\right\}_{1}^{L}$. Namely, let $\zeta_{l} \in C_{0}^{\infty}\left(\mathbb{R}^{d}\right)$ be such that $\sum_{1}^{L} \zeta_{l}(\mathbf{x})=1$ for $\mathbf{x} \in \widetilde{\mathcal{K}}$ and $\operatorname{supp} \zeta_{l} \subset \Omega+\mathbf{x}_{l}^{0}$ for each $l$ and some $\mathbf{x}_{l}^{0} \in \mathbb{R}^{d}$. Then

$$
\int_{\tilde{\mathcal{K}}}\left\langle g \boldsymbol{\psi}, b(\mathbf{D}) \zeta_{l} \mathbf{z}\right\rangle_{\mathbb{C}^{m}} d \mathbf{x}=\int_{\Omega+\mathbf{x}_{l}^{0}}\left\langle g \boldsymbol{\psi}, b(\mathbf{D}) \mathbf{w}_{l}\right\rangle_{\mathbb{C}^{m}} d \mathbf{x}
$$

where $\mathbf{w}_{l}=\zeta_{l} \mathbf{z}$. We extend the restriction of the function $\mathbf{w}_{l}$ to $\Omega+\mathbf{x}_{l}^{0}$ up to a $\Gamma$-periodic function on $\mathbb{R}^{d}$. Then

$$
\int_{\tilde{\mathcal{K}}}\left\langle g \boldsymbol{\psi}, b(\mathbf{D}) \zeta_{l} \mathbf{z}\right\rangle_{\mathbb{C}^{m}} d \mathbf{x}=\int_{\Omega}\left\langle g \boldsymbol{\psi}, b(\mathbf{D}) \mathbf{w}_{l}\right\rangle_{\mathbb{C}^{m}} d \mathbf{x}=0 .
$$

The second identity in (3.29) follows from (3.1.3), because $\left.\mathbf{w}_{l}\right|_{\Omega} \in \widetilde{H}^{1}\left(\Omega ; \mathbb{C}^{n}\right)$. Summing identities (3.29), we arrive at (3.28).

\section{§4. Convergence of solutions and flows (part 2)}

Here we deduce some corollaries to Theorem 3.1, and also analyze the question about conditions under which the convergence of solutions or flows is strong.

4.1. The following theorem is a direct consequence of Theorem 3.1.

Theorem 4.1. Let $\mathbf{u}_{\varepsilon}$ be the solution of equation (3.1), and let $\mathbf{u}_{0}$ be the solution of equation (3.2) with $\mathbf{F} \in \mathfrak{G}^{-1}$. Then:

$1^{\circ}$. As $\varepsilon \rightarrow 0$, the solutions $\mathbf{u}_{\varepsilon}$ converge to $\mathbf{u}_{0}$ weakly in $\mathfrak{G}^{1}$.

$2^{\circ}$. As $\varepsilon \rightarrow 0$, the flows $\mathbf{p}_{\varepsilon}$ converge to the flow $\mathbf{p}_{0}$ weakly in $\mathfrak{G}_{*}$.

4.2. The case where $f \neq \mathbf{1}_{n}$. Consider now the equation

$$
\mathcal{A}_{\varepsilon}(g, f) \widetilde{\mathbf{u}}_{\varepsilon}+\mathfrak{Q}^{\varepsilon} \widetilde{\mathbf{u}}_{\varepsilon}=\mathbf{F}, \quad \mathbf{F} \in \mathfrak{G},
$$

which is more general than (3.1). We call the reader's attention to the fact that the restriction $\mathbf{F} \in \mathfrak{G}$ is imposed in order to lighten the statements. We put $\mathbf{u}_{\varepsilon}=f^{\varepsilon} \widetilde{\mathbf{u}}_{\varepsilon}$, and denote

$$
\mathbf{F}_{\varepsilon}=\left(\left(f^{\varepsilon}\right)^{*}\right)^{-1} \mathbf{F}=\left(\left(f^{*}\right)^{-1}\right)^{\varepsilon} \mathbf{F}, \quad Q=\left(f^{*}\right)^{-1} \mathfrak{Q} f^{-1} .
$$

Then (4.1) turns into the following equation for $\mathbf{u}_{\varepsilon}$ :

$$
\widehat{\mathcal{A}}_{\varepsilon}(g) \mathbf{u}_{\varepsilon}+Q^{\varepsilon} \mathbf{u}_{\varepsilon}=\mathbf{F}_{\varepsilon} .
$$

We apply Theorem 3.1 to this equation. Namely, we put

$$
\mathbf{F}_{0}=\overline{\left(f^{*}\right)^{-1}} \mathbf{F}=\left(\underline{f}^{*}\right)^{-1} \mathbf{F},
$$

and consider the equation

$$
\widehat{\mathcal{A}}\left(g^{0}\right) \mathbf{u}_{0}+\bar{Q} \mathbf{u}_{0}=\mathbf{F}_{0} .
$$

Equations (4.3) and (4.5) satisfy the conditions of Theorem 3.1. Indeed, by (0.2) we have

$$
(w, \mathfrak{G})-\lim _{\varepsilon \rightarrow 0} \mathbf{F}_{\varepsilon}=\mathbf{F}_{0} .
$$

Let $\mathcal{K} \subset \mathbb{R}^{d}$ be a ball. Since the natural embedding $\mathfrak{G} \subseteq H^{-1}\left(\mathcal{K} ; \mathbb{C}^{n}\right)$ is compact, (4.6) implies that

$$
\left(H^{-1}\left(\mathcal{K} ; \mathbb{C}^{n}\right)\right)-\lim _{\varepsilon \rightarrow 0} \mathbf{F}_{\varepsilon}=\mathbf{F}_{0}
$$


So, (3.4) is satisfied. Obviously, the other conditions of Theorem 3.1 are also fulfilled. Thus, we arrive at the following theorem.

Theorem 4.2. Let $\widetilde{\mathbf{u}}_{\varepsilon}$ be the solution of equation (4.1), and let $Q$ and $\mathbf{F}_{0}$ be as in (4.2) and (4.4). Let $\mathbf{u}_{0}$ be the solution of equation (4.5). We put $\widetilde{\mathbf{p}}_{\varepsilon}=g^{\varepsilon} b(\mathbf{D}) f^{\varepsilon} \widetilde{\mathbf{u}}_{\varepsilon}$ and $\mathbf{p}_{0}=g^{0} b(\mathbf{D}) \mathbf{u}_{0}$. Then

$$
\begin{aligned}
\left(w, \mathfrak{G}^{1}\right)-\lim _{\varepsilon \rightarrow 0} f^{\varepsilon} \widetilde{\mathbf{u}}_{\varepsilon} & =\mathbf{u}_{0}, \\
\left(w, \mathfrak{G}_{*}\right)-\lim _{\varepsilon \rightarrow 0} \widetilde{\mathbf{p}}_{\varepsilon} & =\mathbf{p}_{0} .
\end{aligned}
$$

Remark 4.3. Theorem 2.9 can be proved on the basis of Theorem 4.2. We shall not dwell on this.

4.3. About strong convergence. It is of interest to distinguish the cases where the weak limit becomes strong in one of the conclusions of Theorem 4.1.

Theorem 4.4. Under the assumptions of Theorem 4.1, let $\mathbf{F} \in \mathfrak{G}$. Then:

$1^{\circ}$. The $\left(\mathfrak{G}^{1}\right)$-convergence of the solutions $\mathbf{u}_{\varepsilon}$ of $(3.1)$ to the solution $\mathbf{u}_{0}$ of $(3.2)$ is equivalent to the condition

$$
\left(\bar{g}-g^{0}\right) b(\mathbf{D}) \mathbf{u}_{0}=0 .
$$

$2^{\circ}$. The $\left(\mathfrak{G}_{*}\right)$-convergence of the flows $\mathbf{p}_{\varepsilon}=g^{\varepsilon} b(\mathbf{D}) \mathbf{u}_{\varepsilon}$ to the flow $\mathbf{p}_{0}=g^{0} b(\mathbf{D}) \mathbf{u}_{0}$ is equivalent to the condition

$$
\left(g^{0}-\underline{g}\right) b(\mathbf{D}) \mathbf{u}_{0}=0 .
$$

Proof. Both statements are proved similarly. For definiteness, we prove statement $2^{\circ}$. We write

$$
\begin{aligned}
J_{\varepsilon}:= & \left(\left(g^{\varepsilon}\right)^{-1}\left(\mathbf{p}_{\varepsilon}-\mathbf{p}_{0}\right),\left(\mathbf{p}_{\varepsilon}-\mathbf{p}_{0}\right)\right)_{\mathfrak{G}_{*}} \\
= & \left(g^{\varepsilon} b(\mathbf{D}) \mathbf{u}_{\varepsilon}, b(\mathbf{D}) \mathbf{u}_{\varepsilon}\right)_{\mathfrak{G}_{*}}-\left(g^{0} b(\mathbf{D}) \mathbf{u}_{0}, b(\mathbf{D}) \mathbf{u}_{\varepsilon}\right)_{\mathfrak{G}_{*}} \\
& +\left(\left(g^{\varepsilon}\right)^{-1} g^{0} b(\mathbf{D}) \mathbf{u}_{0}, g^{0} b(\mathbf{D}) \mathbf{u}_{0}\right)_{\mathfrak{G}_{*}}-\left(b(\mathbf{D}) \mathbf{u}_{\varepsilon}, g^{0} b(\mathbf{D}) \mathbf{u}_{0}\right)_{\mathfrak{G}_{*}} \\
= & : J_{\varepsilon}^{(1)}-J_{\varepsilon}^{(2)}+J_{\varepsilon}^{(3)}-J_{\varepsilon}^{(4)} .
\end{aligned}
$$

From (3.1) and (3.2) it follows that

$$
\begin{aligned}
J_{\varepsilon}^{(1)} & -J_{\varepsilon}^{(2)}=\left(\bar{Q} \mathbf{u}_{0}-Q^{\varepsilon} \mathbf{u}_{\varepsilon}, \mathbf{u}_{\varepsilon}\right)_{\mathfrak{G}} \\
& =\left(\left(\bar{Q}-Q^{\varepsilon}\right) \mathbf{u}_{0}, \mathbf{u}_{0}\right)_{\mathfrak{G}}+\left(Q^{\varepsilon}\left(\mathbf{u}_{0}-\mathbf{u}_{\varepsilon}\right), \mathbf{u}_{\varepsilon}\right)_{\mathfrak{G}}+\left(\left(\bar{Q}-Q^{\varepsilon}\right) \mathbf{u}_{0}, \mathbf{u}_{\varepsilon}-\mathbf{u}_{0}\right)_{\mathfrak{G}} .
\end{aligned}
$$

By Theorem 2.4, we have $(\mathfrak{G})-\lim _{\varepsilon \rightarrow 0} \mathbf{u}_{\varepsilon}=\mathbf{u}_{0}$. Combining this with (0.2), we obtain

$$
\lim _{\varepsilon \rightarrow 0}\left(J_{\varepsilon}^{(1)}-J_{\varepsilon}^{(2)}\right)=0 \text {. }
$$

Next, by (0.2),

$$
\lim _{\varepsilon \rightarrow 0} J_{\varepsilon}^{(3)}=\left((\underline{g})^{-1} g^{0} b(\mathbf{D}) \mathbf{u}_{0}, g^{0} b(\mathbf{D}) \mathbf{u}_{0}\right)_{\mathfrak{G}_{*}} .
$$

Finally, by statement $1^{\circ}$ of Theorem 4.1 ,

$$
\left(w, \mathfrak{G}_{*}\right)-\lim _{\varepsilon \rightarrow 0} b(\mathbf{D}) \mathbf{u}_{\varepsilon}=b(\mathbf{D}) \mathbf{u}_{0}
$$

whence

$$
\lim _{\varepsilon \rightarrow 0} J_{\varepsilon}^{(4)}=\left(b(\mathbf{D}) \mathbf{u}_{0}, g^{0} b(\mathbf{D}) \mathbf{u}_{0}\right)_{\mathfrak{G}_{*}} .
$$

Relations (4.9)-(4.11) and (4.13) imply

$$
\lim _{\varepsilon \rightarrow 0} J_{\varepsilon}=\left(\left(g^{0}-\underline{g}\right) b(\mathbf{D}) \mathbf{u}_{0},(\underline{g})^{-1} g^{0} b(\mathbf{D}) \mathbf{u}_{0}\right)_{\mathfrak{G}_{*}} .
$$


Consequently, if (4.8) is satisfied, then $J_{\varepsilon} \rightarrow 0$ as $\varepsilon \rightarrow 0$. We conclude that

$$
\left(\mathfrak{G}_{*}\right)-\lim _{\varepsilon \rightarrow 0} \mathbf{p}_{\varepsilon}=\mathbf{p}_{0}
$$

Conversely, suppose that (4.14) is fulfilled for a given $\mathbf{F}$. Then the first summand in the identity $b(\mathbf{D}) \mathbf{u}_{\varepsilon}=\left(g^{\varepsilon}\right)^{-1}\left(\mathbf{p}_{\varepsilon}-\mathbf{p}_{0}\right)+\left(g^{\varepsilon}\right)^{-1} \mathbf{p}_{0}$ tends to zero in $\mathfrak{G}_{*}$. By (0.2), the second summand $\mathfrak{G}_{*}$-weakly converges to $(\underline{g})^{-1} \mathbf{p}_{0}$. Thus,

$$
\left(w, \mathfrak{G}_{*}\right)-\lim _{\varepsilon \rightarrow 0} b(\mathbf{D}) \mathbf{u}_{\varepsilon}=(\underline{g})^{-1} g^{0} b(\mathbf{D}) \mathbf{u}_{0}
$$

Combining this with (4.12), we arrive at (4.8).

Relations (4.7) or (4.8) are valid for any $\mathbf{F} \in \mathfrak{G}$ whenever $g^{0}=\bar{g}$ or $g^{0}=\underline{g}$, respectively. Moreover (see (3.8) and (3.9)), the estimates

$$
\left\|\mathbf{u}_{\varepsilon}\right\|_{\mathfrak{G}^{1}} \leq c_{+}\|\mathbf{F}\|_{\mathfrak{G}^{-1}}, \quad\left\|\mathbf{p}_{\varepsilon}\right\|_{\mathfrak{G}_{*}} \leq \alpha_{1}^{1 / 2} c_{+}\|g\|_{L_{\infty}}\|\mathbf{F}\|_{\mathfrak{G}^{-1}}
$$

allow us to extend the statements about strong convergence to any $\mathbf{F} \in \mathfrak{G}^{-1}$. Thus, we have the following theorem.

Theorem 4.5. Under the assumptions of Theorem 4.1, the following is true.

$1^{\circ}$. If $g^{0}=\bar{g}$, then

$$
\left(\mathfrak{G}^{1}\right)-\lim _{\varepsilon \rightarrow 0} \mathbf{u}_{\varepsilon}=\mathbf{u}_{0}
$$

for any $\mathbf{F} \in \mathfrak{G}^{-1}$.

$2^{\circ}$. If $g^{0}=\underline{g}$, then

$$
\left(\mathfrak{G}_{*}\right)-\lim _{\varepsilon \rightarrow 0} \mathbf{p}_{\varepsilon}=\mathbf{p}_{0}
$$

for any $\mathbf{F} \in \mathfrak{G}^{-1}$. In particular, (4.15) is valid for $m=n$.

In contrast to Theorem 4.4, these sufficient conditions for strong convergence fail to be necessary.

4.4. Theorem 4.5 does not cover all needs of applications. Let $\mathcal{R}$ be yet another $\Gamma$ periodic matrix-valued function such that $\mathcal{R}(\mathbf{x})>0$ and $\mathcal{R}+\mathcal{R}^{-1} \in L_{\infty}$. Consider the equations

$$
\begin{aligned}
\left(\widehat{\mathcal{A}}_{\varepsilon}(g)+Q^{\varepsilon}\right) \mathbf{u}_{\varepsilon} & =\mathcal{R}^{\varepsilon} \mathbf{F}, & & \mathbf{F} \in \mathfrak{G}, \\
\left(\widehat{\mathcal{A}}\left(g^{0}\right)+\bar{Q}\right) \mathbf{u}_{0} & =\overline{\mathcal{R}} \mathbf{F}, & & \mathbf{F} \in \mathfrak{G} .
\end{aligned}
$$

First, we distinguish the following proposition.

Proposition 4.6. We have

$$
(\mathfrak{G})-\lim _{\varepsilon \rightarrow 0} \mathbf{u}_{\varepsilon}=\mathbf{u}_{0} \text {. }
$$

Proof. It suffices to prove (4.18) for $\mathbf{F} \in C_{0}^{\infty}\left(\mathbb{R}^{d} ; \mathbb{C}^{n}\right)$. Let $\mathbf{u}_{\varepsilon}^{\vee}$ be the solution of equation (4.17) with the right-hand side replaced by $\mathcal{R}^{\varepsilon} \mathbf{F}$. By Theorem 2.4, we have $\left\|\mathbf{u}_{\varepsilon}-\mathbf{u}_{\varepsilon}^{\vee}\right\|_{\mathfrak{G}} \rightarrow$ 0 as $\varepsilon \rightarrow 0$. From (0.1) it follows that

$$
(w, \mathfrak{G})-\lim _{\varepsilon \rightarrow 0}\left(\mathcal{R}^{\varepsilon}-\overline{\mathcal{R}}\right) \mathbf{F}=0 .
$$

Let $\eta \in C_{0}^{\infty}\left(\mathbb{R}^{d}\right)$ be such that $\eta \mathbf{F}=\mathbf{F}$. Then

$$
\mathbf{u}_{\varepsilon}^{\vee}-\mathbf{u}_{0}=\left(\left(\widehat{\mathcal{A}}\left(g^{0}\right)+\bar{Q}\right)^{-1} \eta\right)\left(\mathcal{R}^{\varepsilon}-\overline{\mathcal{R}}\right) \mathbf{F} \underset{\varepsilon \rightarrow 0}{\stackrel{\mathfrak{G}}{\longrightarrow}} 0 .
$$

This follows from (4.19) and from the presence of a compact operator (in $\mathfrak{G}$ ) applied to $\left(\mathcal{R}^{\varepsilon}-\overline{\mathcal{R}}\right) \mathbf{F}$. 
Theorem 4.7. Let $\mathbf{u}_{\varepsilon}$ and $\mathbf{u}_{0}$ be the solutions of equations (4.16) and (4.17), respectively. Then:

$1^{\circ}$. If $g^{0}=\bar{g}$, then

$$
\left(\mathfrak{G}^{1}\right)-\lim _{\varepsilon \rightarrow 0} \mathbf{u}_{\varepsilon}=\mathbf{u}_{0}
$$

$2^{\circ}$. If $g^{0}=\underline{g}$, then

$$
\left(\mathfrak{G}_{*}\right)-\lim _{\varepsilon \rightarrow 0} \mathbf{p}_{\varepsilon}=\mathbf{p}_{0}
$$

for the flows (1.3).

We omit the proof, which differs only slightly from that of Theorem 4.4. Note only that again we can assume that $\mathbf{F} \in C_{0}^{\infty}\left(\mathbb{R}^{d} ; \mathbb{C}^{n}\right)$, and that we take (4.18) and (4.19) into account in our estimates.

Now we apply Theorem 4.7 to the case where $f \neq \mathbf{1}_{n}$. Consider equation (4.1):

$$
\mathcal{A}_{\varepsilon}(g, f) \widetilde{\mathbf{u}}_{\varepsilon}+\mathfrak{Q}^{\varepsilon} \widetilde{\mathbf{u}}_{\varepsilon}=\mathbf{F}, \quad \mathbf{F} \in \mathfrak{G} .
$$

We put $\mathbf{u}_{\varepsilon}=f^{\varepsilon} \widetilde{\mathbf{u}}_{\varepsilon}$ and denote

$$
Q=\left(f^{*}\right)^{-1} \mathfrak{Q} f^{-1}, \quad \mathcal{R}=\left(f^{*}\right)^{-1} .
$$

Then (4.20) turns into (4.16). Applying Theorem 4.7 to the latter equation, we arrive at the following theorem.

Theorem 4.8. Let $\widetilde{\mathbf{u}}_{\varepsilon}$ be the solution of equation (4.20), and let $\mathbf{u}_{0}$ be the solution of equation (4.17), where $Q$ and $\mathcal{R}$ are defined by (4.21). Then:

$1^{\circ}$. If $g^{0}=\bar{g}$, then

$$
\left(\mathfrak{G}^{1}\right)-\lim _{\varepsilon \rightarrow 0} f^{\varepsilon} \widetilde{\mathbf{u}}_{\varepsilon}=\mathbf{u}_{0}
$$

$2^{\circ}$. If $g^{0}=\underline{g}$, then, for the flows $\widetilde{\mathbf{p}}_{\varepsilon}=g^{\varepsilon} b(\mathbf{D}) f^{\varepsilon} \widetilde{\mathbf{u}}_{\varepsilon}$, we have

$$
\left(\mathfrak{G}_{*}\right)-\lim _{\varepsilon \rightarrow 0} \widetilde{\mathbf{p}}_{\varepsilon}=\mathbf{p}_{0}=g^{0} b(\mathbf{D}) \mathbf{u}_{0} .
$$

\section{COMMENTS ON Chapter 4}

Here we collect various remarks and comments concerning theorems on homogenization. This material supplements what was said in $\S 1$.

1. The general method of Chapter 1 concerns operators that admit an appropriate factorization. The linear dependence of the factors on the parameter corresponds to the study of second order DO's. Both assumptions, on the one hand, allow us to advance far in analysis, and on the other hand, restrict somewhat the range of applications. However, as can be seen from Chapters 5-7, many important periodic operators of mathematical physics belong to the classes of DO's of the form $\widehat{\mathcal{A}}(g)$ and $\mathcal{A}(g, f)$, which were distinguished in Chapters 2 and 3.

2. In the case of operators of the form $\widehat{\mathcal{A}}_{\varepsilon}(g)$, we deal with homogenization indeed: among the operators that are threshold equivalent to a given one, there exists an operator with constant coefficients. This is directly related to the fact that, for the operator family $\widehat{\mathcal{A}}(\mathbf{k} ; g), \mathbf{k}=t \boldsymbol{\theta}$, the corresponding kernel $\widehat{\mathfrak{N}}$ consists of constants, and the calculation of projections onto $\widehat{\mathfrak{N}}$ is reduced to usual averaging. The class of operators $\widehat{\mathcal{A}}(g)$ (with fixed $b(\mathbf{D})$ ) is closed with respect to homogenization under the resolvent convergence in the operator norm. This is well illustrated by Theorems 2.1 and 2.4.

A different situation arises if we consider a wider class of DO's, namely, $\mathcal{A}(g, f)$. A good approximation for $\left(\mathcal{A}_{\varepsilon}(g, f)+I\right)^{-1}$ is given by another operator family, also having oscillating factors. The choice of such a family is not unique. In this direction, a good result is given by Theorem 2.5, while the result of Theorem 2.3 is less convenient. 
The class of generalized resolvents (2.15) is already closed with respect to homogenization viewed as a weak limit procedure (see Theorem 2.9). This class contains three functional parameters: $g, f$, and $\mathfrak{Q}$. Each of them is "homogenized" by its own rule.

3. Usually, the matrix $b(\mathbf{D})$ is responsible for the type of the physical process in question, while $g$ and $f$ are responsible for its parameters. However, the choice of $b(\mathbf{D})$ is not always unique. For instance (see Subsection 5.2.3 below), the isotropic operator of elasticity theory with constant shear modulus (the Hill body) can be described not only in the universal form, but also via a matrix $b(\mathbf{D})$ of smaller size. This fact leads to useful conclusions.

4. Theorem 2.7 was deduced from Theorem 2.4 with the help of interpolation. Similarly, interpolation could be applied on the basis of Theorem 3.2.3. More specificaly, (3.2.11) could be supplemented by an estimate of the $\left(\mathfrak{G}^{-s} \rightarrow \mathfrak{G}^{s}\right)$-norm of the difference of resolvents for $0 \leq s<1$.

5. Apparently, in homogenization theory, the results like those in $\S 2$ have not been distinguished before. These theorems are the most essential results of the paper both in content and in the method of proof. The corresponding estimates are of precise order, and the constants are well controlled. Theorems 2.1 and 2.3 were published by the authors before in the paper [BSu2]. The other theorems of $\S 2$ are published for the first time. For other possible approaches to such results, see the comments on Chapter 5.

6. The material of $\S \S 3$ and 4 is closer to the usual formulations and techniques of homogenization theory. For instance, the conclusion of Theorem 4.1 looks quite traditional, and in many special cases it leads to known results (see BeLP Sa ZhKO). Here, the representation (3.1.14) for the (main) effective matrix $g^{0}$ is viewed as initial. In the proof of the (main) Theorem 3.1, we, as well as our predecessors, use the periodic solution of the equation on the cell, but only for $\mathbf{k}=0$. Thus, we do not use much of the Floquet decomposition.

Observe that we do not use (at least, explicitly) the method of proof that is usually called a compensated compactness argument (see [BeLP, ZhKO]).

New useful observations are related to Theorems 4.5, 4.7, and 4.8. The corresponding results will be applied in Subsections 5.2.3, 6.2.3, and 6.3.3; see also Remarks 5.1.1 and 6.1.9 and Propositions 7.2.4 and 7.3.8.

Chapter 5. Applications of the general method. The case where $f=\mathbf{1}_{n}$

Here we consider applications of the results of Chapters 3 and 4 to specific periodic operators of mathematical physics in the case where $f=\mathbf{1}_{n}$. We discuss homogenization problems and specific properties of threshold characteristics. The main examples are the acoustic operator, the Schrödinger operator, and the operator of elasticity theory.

\section{§1. The Periodic acoustic and Schrödinger operators}

1.1. The acoustic operator. In $L_{2}\left(\mathbb{R}^{d}\right), d \geq 1$, we consider the operator

$$
\widehat{\mathcal{A}}(g)=\mathbf{D}^{*} g(\mathbf{x}) \mathbf{D}=\nabla^{*} g(\mathbf{x}) \nabla,
$$

which describes a periodic acoustic medium. Here $g(\mathbf{x})$ is a $\Gamma$-periodic $(d \times d)$-matrixvalued function with real entries and such that

$$
g(\mathbf{x})>0, \quad g+g^{-1} \in L_{\infty} .
$$


Now $n=1, m=d \geq 1, b(\xi)=\xi, \mathfrak{H}=L_{2}(\Omega), \mathfrak{H}_{*}=L_{2}\left(\Omega ; \mathbb{C}^{d}\right)$. Obviously, condition (2.1.2) is satisfied. The representation

$$
g(\mathbf{x})=h(\mathbf{x})^{*} h(\mathbf{x})
$$

can be realized, e.g., by putting $h=g^{1 / 2}$. However, the specific choice of a representation as in (1.2) is immaterial. The kernels (3.1.1) and (2.3.5) are given by the relations

$$
\begin{aligned}
\widehat{\mathfrak{N}} & =\{u \in \mathfrak{H}: u=\mathrm{const}\}, \quad d \geq 1, \\
\widehat{\mathfrak{N}}_{*} & =\left\{\mathbf{q} \in \mathfrak{H}_{*}: h^{*} \mathbf{q} \in \widetilde{H}^{1}\left(\Omega ; \mathbb{C}^{d}\right), \operatorname{div} h^{*} \mathbf{q}=0\right\}, \quad n_{*}=\infty, \quad d \geq 2, \\
\widehat{\mathfrak{N}}_{*} & =\left\{q \in \mathfrak{H}_{*}: h^{*} q=\mathrm{const}\right\}, \quad \mathfrak{H}_{*}=\mathfrak{H}, \quad n_{*}=1, \quad d=1 .
\end{aligned}
$$

In accordance with (3.1.5) and (3.1.8), we describe the operators $\widehat{R}(\boldsymbol{\theta}): \widehat{\mathfrak{N}} \rightarrow \widehat{\mathfrak{N}}_{*}$ and $\widehat{S}(\boldsymbol{\theta}): \widehat{\mathfrak{N}} \rightarrow \widehat{\mathfrak{N}}$. Let $v=v(\boldsymbol{\theta}) \in \widetilde{H}^{1}(\Omega)$ be a (weak) solution of the equation $\mathbf{D}^{*} g(\mathbf{D} v+\boldsymbol{\theta})$ $=0$. We put $\mathbf{u}(\boldsymbol{\theta})=h(\mathbf{D} v(\boldsymbol{\theta})+\boldsymbol{\theta}) \in \widehat{\mathfrak{N}}_{*}$. Then $\widehat{R}(\boldsymbol{\theta}) c=c \mathbf{u}(\boldsymbol{\theta}), c \in \widehat{\mathfrak{N}}$, and $\widehat{S}(\boldsymbol{\theta})$ is reduced to multiplication by the number

$$
\widehat{\gamma}(\boldsymbol{\theta})=|\Omega|^{-1}\|\mathbf{u}(\boldsymbol{\theta})\|_{\mathfrak{H}_{*}}^{2}, \quad \boldsymbol{\theta} \in S S^{d-1} .
$$

Now, we construct the effective matrix $g^{0}$ for the operator (1.1). We introduce the functions $v_{j} \in \widetilde{H}^{1}(\Omega), v_{j}=v\left(\mathbf{e}_{j}\right)$, where $\left\{\mathbf{e}_{j}\right\}$ is a fixed basis in $\mathbb{R}^{d}$. Then (see (3.1.12))

$$
g^{0}=|\Omega|^{-1}\left\{\left(\mathbf{u}_{j}, \mathbf{u}_{l}\right)_{\mathfrak{H}_{*}}\right\}, \quad j, l=1, \ldots, d, \quad \mathbf{u}_{j}=h\left(\mathbf{D} v_{j}+\mathbf{e}_{j}\right) .
$$

By Subsection 3.1.3, now the effective matrix $g^{0}$ with real entries is unique. Therefore, it is fair to call $g^{0}$ the matrix of effective medium.

Formula (3.1.8) shows that

$$
\widehat{\gamma}(\boldsymbol{\theta})=\boldsymbol{\theta}^{*} g^{0} \boldsymbol{\theta}=\left\langle g^{0} \boldsymbol{\theta}, \boldsymbol{\theta}\right\rangle_{\mathbb{R}^{d}} .
$$

It is easily seen that the expressions in (1.6)-(1.8) do not depend on the factorization (1.2).

We put

$$
\widehat{\gamma}(\mathbf{k})=t^{2} \widehat{\gamma}(\boldsymbol{\theta})=\left\langle g^{0} \mathbf{k}, \mathbf{k}\right\rangle_{\mathbb{R}^{d}}, \quad \mathbf{k}=t \boldsymbol{\theta} \in \mathbb{R}^{d}, \quad t=|\mathbf{k}| .
$$

Then the effective DO $\widehat{\mathcal{A}}^{0}=\widehat{\mathcal{A}}\left(g^{0}\right)$ can be written as

$$
\widehat{\mathcal{A}}\left(g^{0}\right)=\mathbf{D}^{*} g^{0} \mathbf{D}=\widehat{\gamma}(\mathbf{D}) .
$$

Note that, for $d=1$, we have $m=n(=1)$, whence (see Theorem 3.1.5)

$$
g^{0}=\underline{g} \quad \text { for } d=1 .
$$

By (1.8), if $d=1$, the number $\widehat{\gamma}(\boldsymbol{\theta})$ does not depend on $\boldsymbol{\theta}$, and now only two values $\boldsymbol{\theta}= \pm 1$ are possible.

Theorem 3.2.3 can be applied directly to the operator (1.1). This gives an estimate for the $(\mathfrak{G} \rightarrow \mathfrak{G})$-norm of the difference of resolvents $\left(\widehat{\mathcal{A}}(g)+\varepsilon^{2} I\right)^{-1}-\left(\widehat{\mathcal{A}}\left(g^{0}\right)+\varepsilon^{2} I\right)^{-1}$ for small $\varepsilon>0$; here $\mathfrak{G}=L_{2}\left(\mathbb{R}^{d}\right)$. One can also apply interpolation, as was done in the proof of Theorem 4.2.7. Then we obtain estimates for the $\left(\mathfrak{G}^{-s} \rightarrow \mathfrak{G}^{s}\right)$-norm of the difference of resolvents with $s \in(0,1)$. Such estimates may be of interest for the study of the negative discrete spectrum for the periodic operator $\widehat{\mathcal{A}}(g)$ perturbed by a potential decaying as $|\mathbf{x}| \rightarrow \infty$ ( "admixture" type potential).

To the resolvent of the operator $\widehat{\mathcal{A}}_{\varepsilon}(g)=\widehat{\mathcal{A}}\left(g^{\varepsilon}\right)$, we can apply results about homogenization. Namely, Theorems 4.2.1 and 4.2.7 (the latter theorem with $Q=1$ ) are applicable. Theorems 4.4.1, 4.4.4, and 4.4.5 (again with $Q=1$ ) are also applicable. 
Remark 1.1. Let us dwell on application of Theorem 4.4.5 in more detail. In Subsection 3.1.4 the following known fact (see [ZhKO]) was mentioned. The relation $g^{0}=\bar{g}$ for the acoustic operator is fulfilled if and only if the columns of the matrix $g$ are solenoidal; the relation $g^{0}=\underline{g}$ is fulfilled if and only if the columns of the matrix $g^{-1}$ are potential. Respectively, in the first case the $\left(\mathfrak{G}^{1}\right)$-convergence of the solutions $u_{\varepsilon}$ to $u_{0}$ is strong, and in the second case the $\left(\mathfrak{G}_{*}\right)$-convergence of the flows $\mathbf{p}_{\varepsilon}$ to $\mathbf{p}_{0}$ is strong.

1.2. The Schrödinger operator. Now, we consider the operator

$$
\mathcal{H}(g)=\widehat{\mathcal{A}}(g)+Q(\mathbf{x}),
$$

where $Q(\mathbf{x})>0$ is a $\Gamma$-periodic function such that $Q+Q^{-1} \in L_{\infty}$. The operator $\mathcal{H}(g)$ is a periodic Schrödinger operator (with metric $g$ ). If $g=\mathbf{1}_{d}$, the operator (1.11) is a usual Schrödinger operator for the quantum particle in the external electric field corresponding to the periodic potential $Q$. To the operator

$$
\mathcal{H}_{\varepsilon}(g)=\widehat{\mathcal{A}}_{\varepsilon}(g)+Q^{\varepsilon}(\mathbf{x}),
$$

we can apply Theorems 4.2.4 and 4.2.7 on homogenization. Now, it is convenient to interpret them as estimates for the norm of the difference of the inverses for the operator (1.12) and for the "effective Schrödinger operator"

$$
\mathcal{H}^{0}=\widehat{\mathcal{A}}\left(g^{0}\right)+\bar{Q} .
$$

Theorems 4.4.1, 4.4.4, and 4.4.5 are also applicable, and the analog of Remark 1.1 is true. The operator (1.13) can be viewed as the operator of the free particle in the space with homogeneous metric $g^{0}$. The constant $\bar{Q}$ is responsible for the shift in the energy scale ("renormalization"). Thus, for a rapidly oscillating periodic metric and for electric field, the quantum particle behaves like a free one. The theorems mentioned above give qualitative and quantitative characteristics of the passage of a particle into a free particle, as $\varepsilon \rightarrow 0$. In Subsection 6.1 below, we shall consider another statement of the homogenization problem for the Schrödinger operator.

1.3. Other examples, to which we proceed, will be useful in Subsection 6.2 for the study of the two-dimensional Pauli operator. These examples are also interesting in themselves.

Suppose $d=2, n=m=1$, and let $\omega(\mathbf{x})$ be a $\Gamma$-periodic function such that $\omega(\mathbf{x})>0$ and $\omega+\omega^{-1} \in L_{\infty}$. We consider the pair of operators

$$
\widehat{\mathcal{B}}_{-}\left(\omega^{2}\right)=\partial_{-} \omega^{2}(\mathbf{x}) \partial_{+}, \widehat{\mathcal{B}}_{+}\left(\omega^{2}\right)=\partial_{+} \omega^{2}(\mathbf{x}) \partial_{-}, \quad \partial_{ \pm}:=D_{1} \pm i D_{2} .
$$

The operator $\widehat{\mathcal{B}}_{\mp}\left(\omega^{2}\right)$ is of the form $\widehat{\mathcal{A}}(g)$ with $g=\omega^{2}$ and $b(\boldsymbol{\xi})=\xi^{1} \pm i \xi^{2}$. Since $m=n=1$, the number $g_{\mp}^{0}$ is defined by the formula

$$
g_{-}^{0}=g_{+}^{0}=g^{0}=\underline{\left(\omega^{2}\right)}=\left(|\Omega|^{-1} \int_{\Omega}(\omega(\mathbf{x}))^{-2} d \mathbf{x}\right)^{-1} .
$$

We calculate the germ $\widehat{S}_{\mp}\left(\boldsymbol{\theta} ; \omega^{2}\right)=\widehat{S}_{\mp}\left(\boldsymbol{\theta} ; g^{0}\right)$ of the operator $\widehat{\mathcal{B}}_{\mp}\left(\omega^{2}\right)$. By (3.1.9), we have

$$
\widehat{S}_{\mp}\left(\boldsymbol{\theta} ; \omega^{2}\right)=\left(\theta^{1} \mp i \theta^{2}\right) g^{0}\left(\theta^{1} \pm i \theta^{2}\right)=g^{0} .
$$

Thus, the germs $\widehat{S}_{\mp}$ do not depend on $\theta$, coincide with each other $\left(\widehat{S}_{-}=\widehat{S}_{+}=: \widehat{S}\right)$, and are reduced to multiplication by the constant $\widehat{\gamma}=g^{0}$ defined by (1.15). By (3.1.10), the effective DO $\widehat{\mathcal{B}}_{\mp}^{0}$ for $\widehat{\mathcal{B}}_{\mp}\left(\omega^{2}\right)$ is given by the formula

$$
\widehat{\mathcal{B}}_{\mp}^{0}=\partial_{\mp} g^{0} \partial_{ \pm}=-g^{0} \Delta .
$$

Thus, the effective operators coincide with each other:

$$
\widehat{\mathcal{B}}_{-}^{0}=\widehat{\mathcal{B}}_{+}^{0}=: \widehat{\mathcal{B}}^{0}=-g^{0} \Delta .
$$


The expression (1.16) provides a reason for calling the constant $\left(g^{0}\right)^{-1}$ the effective mass for the operators $\widehat{\mathcal{B}}_{\mp}\left(\omega^{2}\right)$ at the lower edge of the spectrum (i.e., for $\lambda=0$ ).

The operators (1.14) can be treated as the two-dimensional (complex) analogs of the one-dimensional acoustic operator. The fact that $\widehat{S}\left(\omega^{2}\right)$ does not depend on $\boldsymbol{\theta}$ for any $\omega(\mathbf{x})$, bears witness to some hidden symmetry.

Note that the operators (1.14) can also be written more traditionally:

$$
\widehat{\mathcal{B}}_{\mp}\left(\omega^{2}\right)=\mathbf{D}^{*} \omega^{2} \mathfrak{g}_{\mp} \mathbf{D}, \quad \mathfrak{g}_{\mp}=\left(\begin{array}{cc}
1 & \pm i \\
\mp i & 1
\end{array}\right),
$$

but the matrices $\mathfrak{g}_{\mp}$ have one-dimensional kernels.

Consider now the "vector" version of the preceding example, where $d=n=m=2$. Let $\omega_{ \pm}(\mathbf{x})$ be two (real-valued) $\Gamma$-periodic functions such that $\omega_{ \pm}(\mathbf{x})>0$ and $\omega_{ \pm}+\omega_{ \pm}^{-1} \in$ $L_{\infty}$. We put

$$
f_{0}=\operatorname{diag}\left\{\omega_{+}, \omega_{-}\right\} .
$$

In $\mathfrak{G}=L_{2}\left(\mathbb{R}^{2} ; \mathbb{C}^{2}\right)$, we consider the operators

$$
\mathcal{E}=\left(\begin{array}{cc}
0 & \partial_{-} \\
\partial_{+} & 0
\end{array}\right), \quad \widehat{\mathcal{B}}_{\times}=\mathcal{E} f_{0}^{2} \mathcal{E} .
$$

Then

$$
\widehat{\mathcal{B}}_{\times}=\operatorname{diag}\left(\partial_{-} \omega_{-}^{2} \partial_{+}, \partial_{+} \omega_{+}^{2} \partial_{-}\right)=: \operatorname{diag}\left\{\widehat{\mathcal{B}}_{-}\left(\omega_{-}^{2}\right), \widehat{\mathcal{B}}_{+}\left(\omega_{+}^{2}\right)\right\} .
$$

The operator $\widehat{\mathcal{B}}_{\times}$is of the form $\widehat{\mathcal{A}}\left(g_{\times}\right)$with $g_{\times}=f_{0}^{2}$,

$$
b_{\times}(\xi)=\left(\begin{array}{cc}
0 & \xi^{1}-i \xi^{2} \\
\xi^{1}+i \xi^{2} & 0
\end{array}\right) .
$$

The effective matrix is given by the formula

$$
g_{\times}^{0}=\underline{g_{\times}}=\operatorname{diag}\left\{g_{+}^{0}, g_{-}^{0}\right\}, \quad g_{ \pm}^{0}=\underline{\left(\omega_{ \pm}^{2}\right)}
$$

(see (1.15)). Again, the germ $\widehat{S}\left(\boldsymbol{\theta} ; g_{\times}\right)=b_{\times}(\boldsymbol{\theta}) g_{\times}^{0} b_{\times}(\boldsymbol{\theta})$ does not depend on $\boldsymbol{\theta}$ and is reduced to multiplication by the constant diagonal matrix

$$
\widehat{S}\left(\boldsymbol{\theta} ; g_{\times}\right)=\operatorname{diag}\left\{g_{-}^{0}, g_{+}^{0}\right\} .
$$

Finally, now the effective DO $\widehat{\mathcal{B}}_{\times}^{0}$ has the form

$$
\widehat{\mathcal{B}}_{\times}^{0}=\operatorname{diag}\left\{g_{-}^{0}, g_{+}^{0}\right\}(-\Delta) .
$$

As a concluding example, we consider the analog of $\widehat{\mathcal{B}}_{\times}$that involves the metric. Namely, let $\widetilde{g}(\mathbf{x})$ be a $\Gamma$-periodic $(2 \times 2)$-matrix-valued function with real entries and such that $\widetilde{g}(\mathbf{x})>0$ and $\widetilde{g}+\widetilde{g}^{-1} \in L_{\infty}$. Consider the operator

$$
\widehat{\mathcal{B}}_{\times}(g)=\mathcal{E} g \mathcal{E}, \quad g=f_{0} \widetilde{g} f_{0} .
$$

(Of course, we could include the factors $f_{0}$ in $\widetilde{g}$, but in that form the operator (1.22) would be less convenient for application in Subsection 6.2.) The operator (1.22) is of the form $\widehat{\mathcal{A}}(g)$ with $b(\boldsymbol{\xi})=b_{\times}(\boldsymbol{\xi})$ (see $(1.20)$ ). Again, an effective matrix is unique (see Subsection 3.1.3) and can be calculated explicitly:

$$
g^{0}=\underline{g}=\left(|\Omega|^{-1} \int_{\Omega} f_{0}^{-1} \widetilde{g}^{-1} f_{0}^{-1} d \mathbf{x}\right)^{-1} .
$$

Now, the germ $\widehat{S}_{\times}(\boldsymbol{\theta} ; g)=b_{\times}(\boldsymbol{\theta}) \underline{g} b_{\times}(\boldsymbol{\theta})$ depends on $\boldsymbol{\theta}$, but, obviously,

$$
\operatorname{Tr} \widehat{S}_{\times}(\boldsymbol{\theta} ; g)=\operatorname{Tr} \underline{g}, \quad \operatorname{det} \widehat{S}_{\times}(\boldsymbol{\theta} ; g)=\operatorname{det} \underline{g} .
$$


Therefore, the eigenvalues $\gamma_{1}, \gamma_{2}$ of the germ $\widehat{S}_{\times}(\boldsymbol{\theta} ; g)$ do not depend on $\boldsymbol{\theta}$ and coincide with the eigenvalues of the matrix (1.23). The effective operator for the DO (1.22) is given by the general formula (3.1.10):

$$
\widehat{\mathcal{B}}_{\times}\left(g^{0}\right)=\mathcal{E} g^{0} \mathcal{E}
$$

We shall not comment on applications of the general homogenization results to the examples of the present subsection. We only note that, since $m=n(=2)$, Theorem 4.4.5 ensures the strong convergence of the flows for the operator $\mathcal{E} f_{0}^{\varepsilon} \widetilde{g}^{\varepsilon} f_{0}^{\varepsilon} \mathcal{E}$.

\section{$\S 2$. The operator of ElASticity THEORY ON $\mathbb{R}^{d}, d \geq 2$}

2.1. Preliminaries. To represent the operator of elasticity theory in the form $\widehat{\mathcal{A}}=$ $b(\mathbf{D})^{*} g(\mathbf{x}) b(\mathbf{D})$, we need some agreements. Let $\zeta$ be an orthogonal second rank tensor in $\mathbb{R}^{d}$. In a fixed orthonormal basis in $\mathbb{R}^{d}$, it can be represented by a matrix $\zeta=\left\{\zeta_{j l}\right\}_{j, l=1}^{d}$. In the linear space of tensors we introduce the norm $|\zeta|^{2}=\sum_{j, l}\left|\zeta_{j l}\right|^{2}$; this norm does not depend on the choice of the orthonormal basis in $\mathbb{R}^{d}$. We shall consider symmetric tensors, which will be identified with vectors $\zeta_{*} \in \mathbb{C}^{m}, 2 m=d(d+1)$, by the following rule. The vector $\zeta_{*}$ is formed by all components $\zeta_{j l}, j \leq l$, and the pairs $(j, l)$ are put in order in some fixed way. Let $\chi$ be an $(m \times m)$-matrix, $\chi=\operatorname{diag}\left\{\chi_{(j, l)}\right\}$, where $\chi_{(j, l)}=1$ for $j=l$ and $\chi_{(j, l)}=2$ for $j<l$. Then

$$
|\zeta|^{2}=\left\langle\chi \zeta_{*}, \zeta_{*}\right\rangle_{\mathbb{C}^{m}}
$$

For the displacement vector $\mathbf{u} \in \mathfrak{G}^{1}=H^{1}\left(\mathbb{R}^{d} ; \mathbb{C}^{d}\right)$, we introduce the tensors

$$
\nabla \mathbf{u}=\left\{\frac{\partial u_{j}}{\partial x_{l}}\right\}, \quad e(\mathbf{u})=\frac{1}{2}\left\{\frac{\partial u_{j}}{\partial x_{l}}+\frac{\partial u_{l}}{\partial x_{j}}\right\}, \quad r(\mathbf{u})=\frac{1}{2}\left\{\frac{\partial u_{j}}{\partial x_{l}}-\frac{\partial u_{l}}{\partial x_{j}}\right\} .
$$

The deformation tensor $e(\mathbf{u})$ is symmetric. Let $e_{*}(\mathbf{u})$ be the vector corresponding to the tensor $e(\mathbf{u})$ in accordance with the rule described above. The relation

$$
b(\mathbf{D}) \mathbf{u}=-i e_{*}(\mathbf{u})
$$

determines an $(m \times d)$-matrix $b(\mathbf{D})$ uniquely. For instance, with an appropriate ordering, we have

$$
b(\xi)=\left(\begin{array}{cc}
\xi^{1} & 0 \\
\frac{1}{2} \xi^{2} & \frac{1}{2} \xi^{1} \\
0 & \xi^{2}
\end{array}\right), d=2 ; \quad b(\xi)=\left(\begin{array}{ccc}
\xi^{1} & 0 & 0 \\
\frac{1}{2} \xi^{2} & \frac{1}{2} \xi^{1} & 0 \\
0 & \xi^{2} & 0 \\
0 & \frac{1}{2} \xi^{3} & \frac{1}{2} \xi^{2} \\
0 & 0 & \xi^{3} \\
\frac{1}{2} \xi^{3} & 0 & \frac{1}{2} \xi^{1}
\end{array}\right), d=3 .
$$

Now $n=d \geq 2,2 m=d(d+1), \mathfrak{G}=L_{2}\left(\mathbb{R}^{d} ; \mathbb{C}^{d}\right)$, and $\mathfrak{G}_{*}=L_{2}\left(\mathbb{R}^{d} ; \mathbb{C}^{m}\right)$. For $b(\mathbf{D})$ as in (2.2), it is easily seen that $\operatorname{rank} b(\boldsymbol{\xi})=d$ for $\boldsymbol{\xi} \neq 0$, i.e., condition (2.1.2) is satisfied. Now, let $g(\mathbf{x})$ be a $\Gamma$-periodic $(m \times m)$-matrix-valued function with real entries and such that $g(\mathbf{x})>0$ and $g+g^{-1} \in L_{\infty}$, and let

$$
\sigma_{*}(\mathbf{u}):=g(\mathbf{x}) e_{*}(\mathbf{u}) .
$$

The corresponding tensor $\sigma(\mathbf{u})$ is called the stress tensor, and relation (2.3) expresses Hooke's law. The matrix $g$ gives a "concise" description of the Hooke tensor that relates the tensors $\sigma(\mathbf{u})$ and $e(\mathbf{u})$. The matrix $g$ characterizes the parameters of an elastic (in general, anisotropic) medium filling $\mathbb{R}^{d}$. The quadratic form

$$
\mathfrak{w}[\mathbf{u}, \mathbf{u}]=\frac{1}{2} \int_{\mathbb{R}^{d}}\left\langle\sigma_{*}(\mathbf{u}), e_{*}(\mathbf{u})\right\rangle_{\mathbb{C}^{m}} d \mathbf{x}=\frac{1}{2}\left(\sigma_{*}(\mathbf{u}), e_{*}(\mathbf{u})\right)_{\mathfrak{G}_{*}}
$$


gives the energy of elastic deformations. The operator $\mathcal{W}=\mathcal{W}(g)$ of elasticity theory is generated in the space $\mathfrak{G}$ by the quadratic form (2.4). Now, it is clear that $2 \mathcal{W}(g)=\widehat{\mathcal{A}}(g)$, where $g$ is the "Hooke matrix" (see (2.3)), and $b(\mathbf{D})$ is defined by (2.2).

In the case of an isotropic medium, the expression for the form (2.4) simplifies significantly and depends only on two functional Lamé parameters $\lambda(\mathbf{x}), \mu(\mathbf{x})$ :

$$
\begin{aligned}
\mathfrak{w} & {[\mathbf{u}, \mathbf{u}]=\int_{\mathbb{R}^{d}}\left(\mu(\mathbf{x})|e(\mathbf{u})|^{2}+\frac{\lambda(\mathbf{x})}{2}|\operatorname{div} \mathbf{u}|^{2}\right) d \mathbf{x} } \\
& =\int_{\mathbb{R}^{d}}\left(\mu(\mathbf{x})\left\langle\chi e_{*}(\mathbf{u}), e_{*}(\mathbf{u})\right\rangle_{\mathbb{C}^{m}}+\frac{\lambda(\mathbf{x})}{2}|\operatorname{div} \mathbf{u}|^{2}\right) d \mathbf{x} .
\end{aligned}
$$

The parameter $\mu$ is the shear modulus. Often, another parameter $K(\mathbf{x})$ is introduced instead of $\lambda(\mathbf{x}) ; K(\mathbf{x})$ is called the modulus of volume compression. We shall also use yet another modulus $\beta(\mathbf{x})$. Here are the relations:

$$
K(\mathbf{x})=\lambda(\mathbf{x})+\frac{2 \mu(\mathbf{x})}{d}, \quad \beta(\mathbf{x})=\mu(\mathbf{x})+\frac{\lambda(\mathbf{x})}{2} .
$$

The modulus $\lambda(\mathbf{x})$ may be negative. In the isotropic case, the conditions that ensure the positive definiteness of the matrix $g(\mathbf{x})$ are $\mu(\mathbf{x}) \geq \mu_{0}>0, K(\mathbf{x}) \geq K_{0}>0$. We write down the "isotropic" matrices $g$ for $d=2$ and $d=3$ :

$$
\begin{aligned}
g_{\mu, K}(\mathbf{x}) & =\left(\begin{array}{ccc}
K+\mu & 0 & K-\mu \\
0 & 4 \mu & 0 \\
K-\mu & 0 & K+\mu
\end{array}\right), \\
g_{\mu, K}(\mathbf{x})= & \frac{1}{3}\left(\begin{array}{cccccc}
3 K+4 \mu & 0 & 3 K-2 \mu & 0 & 3 K-2 \mu & 0 \\
0 & 12 \mu & 0 & 0 & 0 & 0 \\
3 K-2 \mu & 0 & 3 K+4 \mu & 0 & 3 K-2 \mu & 0 \\
0 & 0 & 0 & 12 \mu & 0 & 0 \\
3 K-2 \mu & 0 & 3 K-2 \mu & 0 & 3 K+4 \mu & 0 \\
0 & 0 & 0 & 0 & 0 & 12 \mu
\end{array}\right), \quad d=3 .
\end{aligned}
$$

All our general results, including those valid only for $f=\mathbf{1}_{n}$, can be applied to the operator $\mathcal{W}(g)$. In particular, the upper and lower estimates (3.1.21) are valid. Note that, in the isotropic case, we have

$$
\overline{g_{\mu, K}}=g_{\bar{\mu}, \bar{K}}, \quad \underline{g_{\mu, K}}=g_{\underline{\mu}, \underline{K}} .
$$

This follows from the fact that the isotropic Hooke tensor is linear with respect to $\mu$ and $K$, and the inverse tensor (see [ZhKO, Chapter 12, §1]) is linear with respect to $\mu^{-1}$ and $K^{-1}$.

2.2. On homogenization for the operator $\mathcal{W}$. Relation (2.3) shows that the flows $\mathbf{p}_{\varepsilon}=g^{\varepsilon} b(\mathbf{D}) \mathbf{u}_{\varepsilon}=-i g^{\varepsilon} e_{*}\left(\mathbf{u}_{\varepsilon}\right)$ corresponding to the operator $2 \mathcal{W}_{\varepsilon}(g)=\widehat{\mathcal{A}}\left(g^{\varepsilon}\right)$ can be viewed as stresses. The effective matrix $g^{0}$ and the effective DO $\mathcal{W}\left(g^{0}\right)$ are constructed in accordance with the general rules. Though the effective matrix $g^{0}$ is not unique now, its choice is determined by the desire to trace the behavior of the stress tensor as $\varepsilon \rightarrow 0$. By Theorem 4.4.1, the solutions $\mathbf{u}_{\varepsilon}$ of the equation

$$
2 \mathcal{W}_{\varepsilon}(g) \mathbf{u}_{\varepsilon}+\mathbf{u}_{\varepsilon}=\mathbf{F}, \quad \mathbf{F} \in \mathfrak{G}^{-1},
$$

$\mathfrak{G}^{1}$-weakly converge to the solution $\mathbf{u}_{0}$ of the equation

$$
2 \mathcal{W}\left(g^{0}\right) \mathbf{u}_{0}+\mathbf{u}_{0}=\mathbf{F}, \quad \mathbf{F} \in \mathfrak{G}^{-1},
$$

and the stresses (the flows) $g^{\varepsilon} e_{*}\left(\mathbf{u}_{\varepsilon}\right)$ converge $\mathfrak{G}_{*}$-weakly to the limit flow $g^{0} e_{*}\left(\mathbf{u}_{0}\right)$, which corresponds to the "limit" stress tensor. For the solutions of equations (2.6) and 
(2.7), these (known) facts are supplemented substantially by applying Theorems 4.2.1 and 4.2.7.

To the more general equation

$$
\left(\mathcal{W}_{\varepsilon}(g)+Q^{\varepsilon}\right) \mathbf{u}_{\varepsilon}=\mathbf{F},
$$

we can also apply Theorems 4.2.4, 4.2.7, and 4.4.1.

No simplification or refinement occurs in the isotropic case; in general, the effective medium is anisotropic.

2.3. The Hill body. In mechanics (see, e.g., [ZhKO]), the isotropic medium with $\mu(\mathbf{x})=\mu_{0}=$ const is called the Hill body. In this case, more economical description of the operator $\mathcal{W}$ is possible, and the homogenization results can be refined. We start with the identity

$$
\int_{\mathbb{R}^{d}}|e(\mathbf{u})|^{2} d \mathbf{x}=\int_{\mathbb{R}^{d}}\left(|r(\mathbf{u})|^{2}+|\operatorname{div} \mathbf{u}|^{2}\right) d \mathbf{x}, \quad \mathbf{u} \in \mathfrak{G}^{1},
$$

where the notation (2.1) is used. Then, for $\mu(\mathbf{x})=\mu_{0}$, the energy form can be represented (by using the notation (2.5)) as

$$
\mathfrak{w}[\mathbf{u}, \mathbf{u}]=\mu_{0} \int_{\mathbb{R}^{d}}|r(\mathbf{u})|^{2} d \mathbf{x}+\int_{\mathbb{R}^{d}} \beta(\mathbf{x})|\operatorname{div} \mathbf{u}|^{2} d \mathbf{x}, \quad \mathbf{u} \in \mathfrak{G}^{1} .
$$

We write the form $(2.8)$ as $\left(g_{\wedge} b_{\wedge}(\mathbf{D}) \mathbf{u}, b_{\wedge}(\mathbf{D}) \mathbf{u}\right)_{\mathfrak{G}_{*}}$ with other $m=m_{\wedge}, g=g_{\wedge}$, and $b=b_{\wedge}$, than those in Subsection 2.1. Namely, let $m_{\wedge}=1+d(d-1) / 2$. The symbol of the $\left(m_{\wedge} \times d\right)$-matrix $b_{\wedge}$ can be described as follows. The first row of $b_{\wedge}(\xi)$ coincides with $\left(\xi^{1}, \xi^{2}, \ldots, \xi^{d}\right)$. The other rows correspond to (different) pairs of indices $(j, l)$, $1 \leq j<l \leq d$. The element standing in the $(j, l)$ th row and the $j$ th column is $\xi^{l}$, and the element in the $(j, l)$ th row and the $l$ th column is $\left(-\xi^{j}\right)$; all other elements of the $(j, l)$ th row are equal to zero. The order of the rows is irrelevant. Finally, $g_{\wedge}(\mathbf{x})$ is defined by the formula

$$
g_{\wedge}(\mathbf{x})=\operatorname{diag}\left\{\beta(\mathbf{x}), \mu_{0} / 2, \mu_{0} / 2, \ldots, \mu_{0} / 2\right\} .
$$

It is easily seen that $\mathfrak{w}[\mathbf{u}, \mathbf{u}]=\left(g_{\wedge} b_{\wedge}(\mathbf{D}) \mathbf{u}, b_{\wedge}(\mathbf{D}) \mathbf{u}\right)_{\mathfrak{G}_{*}}, \mathfrak{G}_{*}^{\wedge}=L_{2}\left(\mathbb{R}^{d} ; \mathbb{C}^{m_{\wedge}}\right)$, or, equivalently,

$$
\mathcal{W}=\left(b_{\wedge}(\mathbf{D})\right)^{*} g_{\wedge}(\mathbf{x}) b_{\wedge}(\mathbf{D}) .
$$

Now we show that

$$
g_{\wedge}^{0}=\underline{g_{\wedge}}=\operatorname{diag}\left\{\underline{\beta}, \mu_{0} / 2, \mu_{0} / 2, \ldots, \mu_{0} / 2\right\} .
$$

For this, we apply Proposition 3.1.7. The assumptions of that proposition need checking only for the first column $\operatorname{col}\left(\beta(\mathbf{x})^{-1}, 0, \ldots, 0\right)$ of the matrix $\left(g_{\wedge}(\mathbf{x})\right)^{-1}$. Let $\vartheta \in \widetilde{H}^{2}(\Omega)$ be a solution of the equation $\Delta \vartheta=i\left(\beta(\mathbf{x})^{-1}-\beta^{-1}\right)$, and let $\mathbf{v}=\nabla \vartheta \in \widetilde{H}^{1}\left(\Omega ; \mathbb{C}^{d}\right)$. Then $\left(b_{\wedge}(\mathbf{D}) \mathbf{v}\right)^{1}=-i \operatorname{div} \mathbf{v}=\beta^{-1}-\underline{\beta}^{-1}$. Obviously, the relations $\left(b_{\wedge}(\mathbf{D}) \mathbf{v}\right)^{j}=0, j>1$, are equivalent to $r(\mathbf{v})=r(\nabla \vartheta)=\overline{0}$. This yields (2.9). Observe that if $\mu(\mathbf{x})=\mu_{0}$, then there is no analog of the identity $g_{\wedge}^{0}=g_{\wedge}$ for the representation of $\mathcal{W}$, which was used in Subsection 2.1. However, in the case of the Hill body, the "usual" effective matrix $g^{0}$ also corresponds to the isotropic medium with parameters $\mu_{0}$ and $\underline{\beta}$.

From (2.9) and Theorem 4.4.5 it follows that, in the homogenization problem for the Hill body, the flows converge strongly. Namely, let $\mathbf{u}_{\varepsilon}$ be the solution of the equation

$$
\left(b_{\wedge}(\mathbf{D})\right)^{*} g_{\wedge}^{\varepsilon} b_{\wedge}(\mathbf{D}) \mathbf{u}_{\varepsilon}+\mathbf{u}_{\varepsilon}=\mathbf{F}, \quad \mathbf{F} \in \mathfrak{G}^{-1},
$$

and let $\mathbf{u}_{0}$ be the solution of the equation

$$
\left(b_{\wedge}(\mathbf{D})\right)^{*} g_{\wedge}^{0} b_{\wedge}(\mathbf{D}) \mathbf{u}_{0}+\mathbf{u}_{0}=\mathbf{F} .
$$


Then

$$
\left(\mathfrak{G}_{*}^{\wedge}\right)-\lim _{\varepsilon \rightarrow 0} g_{\wedge}^{\varepsilon} b_{\wedge}(\mathbf{D}) \mathbf{u}_{\varepsilon}=g_{\wedge}^{0} b_{\wedge}(\mathbf{D}) \mathbf{u}_{0}
$$

Clearly, (2.10) is equivalent to the pair of relations

$$
\begin{aligned}
\left(L_{2}\left(\mathbb{R}^{d}\right)\right)-\lim _{\varepsilon \rightarrow 0} \beta^{\varepsilon} \operatorname{div} \mathbf{u}_{\varepsilon} & =\underline{\beta} \operatorname{div} \mathbf{u}_{0}, \\
\left(L_{2}\left(\mathbb{R}^{d}\right)\right)-\lim _{\varepsilon \rightarrow 0} r\left(\mathbf{u}_{\varepsilon}\right) & =r\left(\mathbf{u}_{0}\right) .
\end{aligned}
$$

However, this is less than the strong convergence of the full stress tensor.

\section{Comments on Chapter 5}

1. For all three main examples (the acoustic operator, the Schrödinger operator, and the operator of elasticity theory), application of Theorems 4.2.1, 4.2.4, 4.2.7 to the homogenization problem yields new results (partly, these results were published by the authors in [BSu2]). In contrast, application of Theorem 4.4.1 to these cases gives well known statements. Relations (2.11) and (2.12) concerning strong convergence for the Hill body have not been mentioned before.

2. The examples in Subsection 1.3 are given by way of illustration; they also prepare the study of the two-dimensional periodic Pauli operator in $\S 6.2$. In many respects, the operators $\widehat{\mathcal{B}}_{ \pm}\left(\omega^{2}\right)$ (see (1.14)) are similar to the acoustic operator with $d=1$. In particular, formulas (1.15) have the same form as (1.10).

3. For the acoustic and Schrödinger operators, we have $n=1$. Then the only eigenvalue $\gamma(\mathbf{k})$ of the operator $\widehat{\mathcal{A}}(\mathbf{k}, g)$ acting in $L_{2}(\Omega)$ (and corresponding to the DO (1.1)) is analytic near the point $\mathbf{k}=0$. The same is true for the corresponding eigenfunction. On this basis, for instance, an estimate of the type

$$
\left\|\left(\widehat{\mathcal{A}}_{\varepsilon}(g)+I\right)^{-1}-\left(\widehat{\mathcal{A}}\left(g^{0}\right)+I\right)^{-1}\right\|_{\mathfrak{G} \rightarrow \mathfrak{G}} \leq \text { (const) } \varepsilon
$$

can be proved in a more direct way. For this, the Floquet decomposition should be employed, but there is no need to use general results of Chapters 1 and 3. Similar methods were applied earlier for the study of the Schrödinger operator perturbed by potentials of admixture type (see [B1, B2,,$\overline{B L a S u}]$ ). However, it is more difficult to control the constant in $(*)$ under this approach.

4. In the paper [Zh1] (see also [ZhKO, II.6]), Zhikov used the Floquet theory to obtain meaningful estimates for the fundamental solution of the parabolic equation corresponding to the DO (1.1). In a discussion of the results of [BSu2], Zhikov [Zh2] communicated to the authors that an estimate of the form $(*)$ can be deduced from estimates for the fundamental solution in a relatively easy way. This can be done on the basis of [Zh1], but some smoothness assumptions on $g$ are required, and it is difficult to control the constants in estimates.

\section{Chapter 6. Applications of the general method. The case where $f \neq \mathbf{1}_{n}$}

In this case, the general results are less perfect, although even these results allow us to advance essentially at a number of points. In particular, this concerns the homogenization problems. Our main examples are the Schrödinger operator and the two-dimensional Pauli operator. Both operators can be represented as $\mathcal{A}(g, f)$, by appropriate factorization. Finally, we consider the homogenization problem for a certain first order DO. 


\section{§1. THE PERIOdIC SCHRÖDINGER OPERATOR}

1.1. Preliminaries. Factorization. In the space $L_{2}\left(\mathbb{R}^{d}\right), d \geq 1$, we consider the periodic Schrödinger operator (with metric) of the form

$$
\mathcal{H}=\mathbf{D}^{*} \widetilde{g}(\mathbf{x}) \mathbf{D}+p(\mathbf{x}), \quad \mathbf{x} \in \mathbb{R}^{d},
$$

where a $(d \times d)$-matrix $\widetilde{g}(\mathbf{x})>0$ with real entries and a real-valued potential $p(\mathbf{x})$ are $\Gamma$-periodic and such that

$$
\begin{gathered}
\widetilde{g}+\widetilde{g}^{-1} \in L_{\infty}, \\
p \in L_{s}(\Omega), \quad \text { where } 2 s>d \text { for } d \geq 2 \text { and } s=1 \text { for } d=1 .
\end{gathered}
$$

The precise definition of the selfadjoint operator $\mathcal{H}$ in $\mathfrak{G}=L_{2}\left(\mathbb{R}^{d}\right)$ is given in terms of the quadratic form

$$
\mathfrak{h}[u, u]=\int_{\mathbb{R}^{d}}\left(\langle\widetilde{g} \mathbf{D} u, \mathbf{D} u\rangle_{\mathbb{C}^{d}}+p|u|^{2}\right) d \mathbf{x}, \quad u \in \mathfrak{G}^{1}=H^{1}\left(\mathbb{R}^{d}\right) .
$$

Under conditions (1.2) and (1.3), this form is lower semibounded and closed in $\mathfrak{G}$. Adding an appropriate constant to $p$, we can assume that the point $\lambda=0$ is the lower edge of the spectrum of $\mathcal{H}$. The latter condition is assumed in the sequel.

We consider the homogeneous equation $\left(\mathbf{D}^{*} \widetilde{g} \mathbf{D}+p\right) \omega=0$, which is understood in the sense of the following identity for $\omega \in \widetilde{H}^{1}(\Omega)$ :

$$
\int_{\Omega}\left(\langle\widetilde{g} \mathbf{D} \omega, \mathbf{D} z\rangle_{\mathbb{C}^{d}}+p \omega z^{+}\right) d \mathbf{x}=0, \quad z \in \widetilde{H}^{1}(\Omega) .
$$

Under conditions (1.2) and (1.3), this equation has a positive $\Gamma$-periodic solution $\omega$ that belongs to $H_{\text {loc }}^{1}\left(\mathbb{R}^{d}\right) \cap \operatorname{Lip} \tau$ (for some $\tau>0$ ). Moreover, the function $\omega$ is a multiplier in the classes $\mathfrak{G}^{1}$ and $\widetilde{H}^{1}(\Omega)$. After the substitution $u=\omega v$, the form (1.4) turns into

$$
\mathfrak{h}[u, u]=\int_{\mathbb{R}^{d}} \omega^{2}\langle\widetilde{g} \mathbf{D} v, \mathbf{D} v\rangle_{\mathbb{C}^{d}} d \mathbf{x}, \quad u=\omega v, \quad v \in \mathfrak{G}^{1} .
$$

This means that the operator (1.1) is represented as a product

$$
\mathcal{H}=\omega^{-1} \mathbf{D}^{*} \omega^{2} \widetilde{g} \mathbf{D} \omega^{-1} .
$$

Thus, the operator $\mathcal{H}$ takes the form

$$
\mathcal{H}=\mathcal{A}(g, f), \quad g=\omega^{2} \widetilde{g}, \quad f=\omega^{-1},
$$

with $n=1, m=d \geq 1, b(\xi)=\xi$.

Remark 1.1. Expression (1.6) can be taken as the definition of the operator $\mathcal{H}$ for any measurable $\Gamma$-periodic function $\omega$ such that

$$
\omega(\mathbf{x})>0, \quad \omega+\omega^{-1} \in L_{\infty} .
$$

The form (1.1) can be recovered by the formula $p=-\omega^{-1}\left(\mathbf{D}^{*} \widetilde{g} \mathbf{D} \omega\right)$. The corresponding potential $p$ may be strongly singular.

Remark 1.2. Let $E_{j}(\mathbf{k})$ be the band functions of the periodic operator (1.1). The representation (1.7) implies that $E_{1}(\mathbf{k})$ has a nondegenerate minimum (equal to zero) at $\mathbf{k}=0$ and that $\min E_{2}(\mathbf{k})>0$. These properties follow from the existence of a positive periodic solution of equation (1.5). In fact, any other way of establishing these properties of the band functions is equivalent to the proof of the existence of a positive solution $\omega$. 
1.2. Relationship with the acoustic operator. The operator $\widehat{\mathcal{A}}(g)$ corresponding to the operator (1.7) with $f=1$ coincides with the acoustic operator (5.1.1). Thus, we are under the conditions of the general pattern of Subsection 1.1.5 with $M=M^{*}=\omega^{-1}$. In accordance with $(5.1 .3), \mathfrak{H}=L_{2}(\Omega)$ and

$$
\mathfrak{N}=\{u \in \mathfrak{H}: u=c \omega, c \in \mathbb{C}\},
$$

while $\mathfrak{N}_{*}=\widehat{\mathfrak{N}}_{*}$ is defined by (5.1.4) and (5.1.5). By definition, the effective matrix $g^{0}$ for the operator (1.7) is the same as for the acoustic operator. For the latter, since $n=1$, the germ $\widehat{S}(\boldsymbol{\theta})$ acting in $\widehat{\mathfrak{N}}$ is reduced to multiplication by the number

$$
\widehat{\gamma}(\boldsymbol{\theta})=\left\langle g^{0} \boldsymbol{\theta}, \boldsymbol{\theta}\right\rangle_{\mathbb{R}^{d}}
$$

(see (5.1.8)). For calculating the germ $S(\boldsymbol{\theta})$ of the operator $\mathcal{H}$, we use formula (1.1.26). Now $M=\omega^{-1}$, and we can put $\zeta=\omega$ (see (1.8)). Then (1.1.26) shows that $S(\boldsymbol{\theta})$ acts as multiplication of the elements of the kernel (1.8) by the number

$$
\gamma(\boldsymbol{\theta})=\widehat{\gamma}(\boldsymbol{\theta})|\Omega|\|\omega\|_{\mathfrak{H}}^{-2}, \quad \boldsymbol{\theta} \in S S^{d-1} .
$$

We normalize the choice of $\omega$ by the condition

$$
|\Omega|^{-1} \int_{\Omega} \omega^{2} d \mathbf{x}=1
$$

Then (1.10) leads to the following useful statement.

Proposition 1.3. Suppose a positive solution $\omega$ of equation (1.5) is normalized as in (1.11), and the matrix $g$ is defined by (1.7). Then

$$
\gamma(\boldsymbol{\theta})=\widehat{\gamma}(\boldsymbol{\theta})=\left\langle g^{0} \boldsymbol{\theta}, \boldsymbol{\theta}\right\rangle_{\mathbb{R}^{d}}, \quad \boldsymbol{\theta} \in S S^{d-1} .
$$

Remark 1.4. In quantum mechanics, the tensor inverse to the tensor $\gamma$ is called the tensor of effective masses. Thus, (1.12) means that, under condition (1.11) and for $g=\widetilde{g} \omega^{2}$, the tensors of effective masses for the operators (1.1) and (5.1.1) coincide.

By our definitions, under condition (1.11) and for $g=\widetilde{g} \omega^{2}$, the effective DO for the operator $\mathcal{H}$ can be written as

$$
(\omega(\mathbf{x}))^{-1} \widehat{\gamma}(\mathbf{D})(\omega(\mathbf{x}))^{-1},
$$

where the tensor $\widehat{\gamma}$ (see (1.9)) is related to the corresponding acoustic operator.

We distinguish the case where $d=1$. Then $m=n=1$ and (see (5.1.10)) $\widehat{\gamma}=g^{0}=\underline{g}$. Combining this with (1.10), we obtain

$$
\gamma=|\Omega|^{2}\|\omega\|_{\mathfrak{H}}^{-2}\left\|\widetilde{g}^{-1 / 2} \omega^{-1}\right\|_{\mathfrak{H}}^{-2}, \quad d=1 .
$$

In particular,

$$
\gamma=|\Omega|^{2}\|\omega\|_{\mathfrak{H}}^{-2}\left\|\omega^{-1}\right\|_{\mathfrak{H}}^{-2} \quad \text { for } d=1, \widetilde{g}=1 .
$$

The latter formula is well known in quantum mechanics as the formula for the effective mass $\gamma^{-1}$ on the left edge of the spectrum.

1.3. Homogenization. We consider the family of operators $\mathcal{H}_{\varepsilon}$ that are defined, in accordance with (1.6) and (1.7), by the formulas

$$
\mathcal{H}_{\varepsilon}=\mathcal{H}_{\varepsilon}(\widetilde{g}, \omega)=\left(\omega^{\varepsilon}\right)^{-1} \mathbf{D}^{*} g^{\varepsilon} \mathbf{D}\left(\omega^{\varepsilon}\right)^{-1}, \quad g^{\varepsilon}=\left(\omega^{2} \widetilde{g}\right)^{\varepsilon}, \quad \varepsilon>0 .
$$

Also, this definition can be rewritten in the initial terms:

$$
\mathcal{H}_{\varepsilon}=\mathbf{D}^{*}(\widetilde{g})^{\varepsilon} \mathbf{D}+\varepsilon^{-2} p^{\varepsilon} .
$$

The family (1.15) differs from (5.1.12): now the potential $p$ is certainly not sign-definite. Moreover, $p^{\varepsilon}$ is multiplied by $\varepsilon^{-2}$, which "equalizes" the roles of the summands in (1.15). 
In homogenization of the family (5.1.12), the potential term has a smaller impact on the result.

To the operator $\mathcal{H}_{\varepsilon}$ we can apply all the results of general nature for the family $\mathcal{A}_{\varepsilon}(g, f)$ with variable $f$. We formulate some consequences of the general results. We shall not write down the constants in estimates explicitly, though this can easily be done: the general formulas for the constants may only simplify. All constants in estimates in this subsection will be denoted by the letter $C$ without indices.

First, we apply Theorem 4.2.5.

Theorem 1.5. Suppose $g=\widetilde{g} \omega^{2}$ and (1.11) is satisfied. Let $\widehat{\mathcal{H}}\left(g^{0}\right)$ denote the operator $\mathbf{D}^{*} g^{0} \mathbf{D}$. Then

$$
\left\|\left(\mathcal{H}_{\varepsilon}(\widetilde{g}, \omega)+I\right)^{-1}-\omega^{\varepsilon}\left(\widehat{\mathcal{H}}\left(g^{0}\right)+I\right)^{-1} \omega^{\varepsilon}\right\|_{\mathfrak{G} \rightarrow \mathfrak{G}} \leq C \varepsilon, \quad 0<\varepsilon \leq 1 .
$$

Proof. It suffices to refer to (3.3.2) and the normalization condition (1.11). As a result, we obtain $\bar{Q}=1$.

This estimate can be supplemented with the interpolation estimate from Theorem 4.2.8.

Theorem 1.6. Under the assumptions of Theorem 1.5, we have

$$
\left\|\left(\omega^{\varepsilon}\right)^{-1}\left(\mathcal{H}_{\varepsilon}(\widetilde{g}, \omega)+I\right)^{-1}-\left(\widehat{\mathcal{H}}\left(g^{0}\right)+I\right)^{-1} \omega^{\varepsilon}\right\|_{\mathfrak{G} \rightarrow \mathfrak{G}^{s}} \leq C \varepsilon^{1-s}, \quad 0 \leq s<1, \quad 0<\varepsilon \leq 1 .
$$

Next, Theorem 4.2.9 (with $\mathfrak{Q}=1$ ) implies the following result.

Theorem 1.7. Under the assumptions of Theorem 1.5, we have

$$
(w, \mathfrak{G} \rightarrow \mathfrak{G})-\lim _{\varepsilon \rightarrow 0}\left(\mathcal{H}_{\varepsilon}(\widetilde{g}, \omega)+I\right)^{-1}=(\bar{\omega})^{2}\left(\widehat{\mathcal{H}}\left(g^{0}\right)+I\right)^{-1} .
$$

Finally, we mention the following consequence of Theorem 4.4.2 (again with $\mathfrak{Q}=1$ ). Let $\widetilde{u}_{\varepsilon}$ be the solution of the equation

$$
\left(\mathcal{H}_{\varepsilon}(\widetilde{g}, \omega)+I\right) \widetilde{u}_{\varepsilon}=F, \quad F \in \mathfrak{G} .
$$

Suppose that (1.11) is satisfied and $g=\widetilde{g} \omega^{2}$. Let $u_{0}$ be the solution of the equation

$$
\left(\widehat{\mathcal{H}}\left(g^{0}\right)+I\right) u_{0}=\bar{\omega} F .
$$

Theorem 1.8. Under the above assumptions,

$$
\begin{aligned}
\left(w, \mathfrak{G}^{1}\right)-\lim _{\varepsilon \rightarrow 0}\left(\omega^{\varepsilon}\right)^{-1} \widetilde{u}_{\varepsilon} & =u_{0}, \\
\left(w, \mathfrak{G}_{*}\right)-\lim _{\varepsilon \rightarrow 0} g^{\varepsilon} \mathbf{D}\left(\left(\omega^{\varepsilon}\right)^{-1} \widetilde{u}_{\varepsilon}\right) & =g^{0} \mathbf{D} u_{0} .
\end{aligned}
$$

By Theorem 4.4.8 and Propositions 3.1.6 and 3.1.7, the following analog of Remark 5.1.1 is valid for the Schrödinger operator (1.1).

Remark 1.9. If the columns of the matrix $g=\omega^{2} \widetilde{g}$ are solenoidal, convergence in (1.17) is strong. If the columns of the matrix $g^{-1}=\omega^{-2} \widetilde{g}^{-1}$ are potential, convergence in (1.18) is strong.

\section{§2. The two-dimensional periodic Pauli operator}

2.1. Definition and factorization of the Pauli operator. The role of the magnetic potential will be played by a $\Gamma$-periodic $\mathbb{R}^{2}$-valued function $\mathbf{A}=\left\{A_{1}, A_{2}\right\}$ on $\mathbb{R}^{2}$ such that

$$
\mathbf{A} \in L_{\rho}\left(\Omega ; \mathbb{C}^{2}\right), \quad \rho>2 .
$$


Recall the standard notation for the Pauli matrices:

$$
\sigma_{1}=\left(\begin{array}{ll}
0 & 1 \\
1 & 0
\end{array}\right), \quad \sigma_{2}=\left(\begin{array}{cc}
0 & -i \\
i & 0
\end{array}\right), \quad \sigma_{3}=\left(\begin{array}{cc}
1 & 0 \\
0 & -1
\end{array}\right) .
$$

In the space $\mathfrak{G}=L_{2}\left(\mathbb{R}^{2} ; \mathbb{C}^{2}\right)$ we consider the operator

$$
\mathcal{D}:=\left(D_{1}-A_{1}\right) \sigma_{1}+\left(D_{2}-A_{2}\right) \sigma_{2}, \quad \operatorname{Dom} \mathcal{D}=\mathfrak{G}^{1}=H^{1}\left(\mathbb{R}^{2} ; \mathbb{C}^{2}\right) .
$$

By definition, the Pauli operator $\mathcal{P}$ is the square of the operator $\mathcal{D}$ :

$$
\mathcal{P}:=\mathcal{D}^{2}=\left(\begin{array}{cc}
P_{-} & 0 \\
0 & P_{+}
\end{array}\right)
$$

The precise definition of $\mathcal{P}$ is given via the closed quadratic form

$$
\|\mathcal{D} \mathbf{u}\|_{\mathfrak{G}}^{2}, \quad \mathbf{u} \in \operatorname{Dom} \mathcal{D}
$$

in $\mathfrak{G}$. If the potential $\mathbf{A}$ is sufficiently smooth, the blocks $P_{ \pm}$of the operator $(2.3)$ can be written as

$$
P_{ \pm}=(\mathbf{D}-\mathbf{A})^{2} \pm\left(\partial_{1} A_{2}-\partial_{2} A_{1}\right)
$$

The expression $\partial_{1} A_{2}-\partial_{2} A_{1}$ corresponds to the strength of the magnetic field.

We use the known factorization for the operators (2.2) and (2.3) (see [BSu1] 2$]$ for the details). A gauge transformation allows us to assume that the potential $\mathbf{A}$ is subject to the requirements

$$
\operatorname{div} \mathbf{A}=0, \quad \int_{\Omega} \mathbf{A} d \mathbf{x}=0
$$

and still satisfies (2.1). Under conditions (2.1) and (2.6), there exists a (unique) realvalued $\Gamma$-periodic function $\varphi$ such that

$$
\nabla \varphi=\left\{A_{2},-A_{1}\right\}, \quad \bar{\varphi}=0 .
$$

From (2.1) and (2.7) it follows that

$$
\varphi \in \widetilde{W}_{\rho}^{1}(\Omega) \subset \operatorname{Lip} \tau, \quad \tau=1-2 \rho^{-1} .
$$

Here $\widetilde{W}_{\rho}^{1}(\Omega)$ is the subspace of the Sobolev space $W_{\rho}^{1}(\Omega)$ formed by the functions whose $\Gamma$-periodic extension belongs to $W_{\rho, \text { loc }}^{1}\left(\mathbb{R}^{2}\right)$.

We introduce the notation (cf. (5.1.14), (5.1.17), and (5.1.18))

$$
\omega_{ \pm}:=\exp ( \pm \varphi), \quad f_{0}=\left(\begin{array}{cc}
\omega_{+} & 0 \\
0 & \omega_{-}
\end{array}\right), \quad \mathcal{E}=\left(\begin{array}{cc}
0 & \partial_{-} \\
\partial_{+} & 0
\end{array}\right) .
$$

By $(2.8)$, we have $\omega_{ \pm} \in \widetilde{W}_{\rho}^{1}(\Omega)$ and

$$
\omega_{-}(\mathbf{x}) \omega_{+}(\mathbf{x})=1, \quad \mathbf{x} \in \mathbb{R}^{2} .
$$

The operators (2.2) and (2.3) can be written as

$$
\begin{aligned}
& \mathcal{D}=f_{0} \mathcal{E} f_{0}, \\
& \mathcal{P}=f_{0} \mathcal{E} f_{0}^{2} \mathcal{E} f_{0} .
\end{aligned}
$$

The blocks (2.5) of the operator (2.3) admit the representations

$$
P_{+}=Y^{*} Y, P_{-}=Y Y^{*}, \quad \text { where } Y:=\omega_{+} \partial_{-} \omega_{-} .
$$

It is convenient to view formulas $(2.11)-(2.13)$ as the definitions of the operators $\mathcal{D}$, $\mathcal{P}$, and $P_{ \pm}$, assuming that the $\omega_{ \pm}$are arbitrary $\Gamma$-periodic functions satisfying $(2.10)$ and such that

$$
\omega_{ \pm}(\mathbf{x})>0, \quad \omega_{+}, \omega_{-} \in L_{\infty}
$$


More precisely, the operator $\mathcal{D}$ is given by (2.11) on the domain

$$
\operatorname{Dom} \mathcal{D}=\left\{\mathbf{u} \in \mathfrak{G}: f_{0} \mathbf{u} \in \mathfrak{G}^{1}\right\} .
$$

The operator $\mathcal{P}$ corresponds to the quadratic form (2.4) defined on the domain (2.14). The blocks $P_{ \pm}$are defined via the quadratic forms

$$
\left\|\omega_{ \pm} \partial_{\mp} \omega_{\mp} u\right\|_{L_{2}\left(\mathbb{R}^{2}\right)}^{2}, \quad \omega_{\mp} u \in H^{1}\left(\mathbb{R}^{2}\right) .
$$

Here, the magnetic field strength in (2.5) loses its immediate meaning.

Clearly, $\operatorname{Ker} Y=\operatorname{Ker} Y^{*}=\{0\}$. Therefore, (2.13) implies that the operator $P_{+}$is unitarily equivalent to $P_{-}$. Obviously, the study of the operator $\mathcal{P}$ reduces to the study of the blocks $P_{ \pm}$.

2.2. Effective masses and DO. The operators $P_{ \pm}$and $\mathcal{P}$ fall into the general pattern.

We start with $P_{ \pm}$. Now, we have $d=2, m=n=1, \mathfrak{G}=\mathfrak{G}_{*}=L_{2}\left(\mathbb{R}^{2}\right)$, and $\mathfrak{H}=\mathfrak{H}_{*}=L_{2}(\Omega)$. The corresponding kernels are given by the formulas

$$
\mathfrak{N}_{ \pm}=\left\{u \in \mathfrak{H}: u=c \omega_{ \pm}, c \in \mathbb{C}\right\}, \quad\left(\mathfrak{N}_{*}\right)_{ \pm}=\mathfrak{N}_{\mp}, \quad n=n_{*}=1 .
$$

The operators $P_{ \pm}$can be represented as $\mathcal{A}(g, f)$. Namely, the roles of $g, f$, and $b(\boldsymbol{\xi})$ are played by $\omega_{ \pm}^{2}, \omega_{\mp}$, and $\xi^{1} \mp i \xi^{2}$, respectively. Then $P_{ \pm}=\mathcal{A}\left(\omega_{ \pm}^{2}, \omega_{\mp}\right)$, and the role of the corresponding operator $\widehat{\mathcal{A}}(g)$ is played by $\widehat{\mathcal{B}}_{ \pm}\left(\omega_{ \pm}^{2}\right)$ (see (5.1.14)). Consequently (see (5.1.15)), the effective matrix $g_{ \pm}^{0}$ reduces to the number

$$
g_{ \pm}^{0}=\left(|\Omega|^{-1} \int_{\Omega} \omega_{\mp}^{2} d \mathbf{x}\right)^{-1}
$$

Accordingly (see (5.1.16)), the effective DO is given by the formula

$$
P_{ \pm}^{0}=-g_{ \pm}^{0} \omega_{\mp}(\mathbf{x}) \Delta \omega_{\mp}(\mathbf{x}) .
$$

In order to calculate the germ $S_{ \pm}(\boldsymbol{\theta})$ of $P_{ \pm}$, we use (3.1.30). By this formula, $S_{ \pm}(\boldsymbol{\theta})$ reduces to multiplication by the number $\gamma_{ \pm}(\boldsymbol{\theta})=\widehat{\gamma}_{ \pm}(\boldsymbol{\theta})|\Omega|\left\|\omega_{ \pm}\right\|_{\mathfrak{H}}^{-2}$. Now, the relation $\widehat{\gamma}(\boldsymbol{\theta})=g^{0}$ in Subsection 5.1.3, applied to $\widehat{\mathcal{B}}_{ \pm}\left(\omega_{ \pm}^{2}\right)$, shows that $\widehat{\gamma}_{ \pm}(\boldsymbol{\theta})=g_{ \pm}^{0}$. By $(2.10)$ and (2.15), we obtain

$$
\gamma_{ \pm}(\theta)=|\Omega|^{2}\left\|\omega_{-}\right\|_{\mathfrak{H}}^{-2}\left\|\omega_{+}\right\|_{\mathfrak{H}}^{-2}=: \gamma .
$$

Thus, the numbers $\gamma_{ \pm}(\boldsymbol{\theta})$ do not depend on $\boldsymbol{\theta}$ and coincide with each other. Formulas (2.17) are full analogs of formula (1.13) for the one-dimensional Schrödinger operator. Herewith, the effective masses for the two Pauli operators $P_{ \pm}$coincide with each other and do not depend on $\boldsymbol{\theta}$.

We say a few words about the operator $\mathcal{P}$. Here $m=n=d=2$, and $\mathfrak{G}=\mathfrak{G}_{*}=$ $L_{2}\left(\mathbb{R}^{2} ; \mathbb{C}^{2}\right)$. The operator $\mathcal{P}$ is of the form $\mathcal{A}(g, f)$, where $g=f_{0}^{2}, f=f_{0}$, and $b(\xi)$ is defined by (5.1.20). The role of the corresponding operator $\widehat{\mathcal{A}}(g)$ is played by the operator $\widehat{\mathcal{B}}_{\times}$defined by (5.1.19). In accordance with (3.1.29) and (5.1.21), the effective DO $\mathcal{P}^{0}$ for the operator $\mathcal{P}$ looks like this:

$$
\mathcal{P}^{0}=f_{0}(\mathbf{x}) \widehat{\mathcal{B}}_{\times}^{0} f_{0}(\mathbf{x})=f_{0}(\mathbf{x})\left(\begin{array}{cc}
-g_{-}^{0} \Delta & 0 \\
0 & -g_{+}^{0} \Delta
\end{array}\right) f_{0}(\mathbf{x})=\left(\begin{array}{cc}
P_{-}^{0} & 0 \\
0 & P_{+}^{0}
\end{array}\right) .
$$

Thus, $\mathcal{P}^{0}$ is expressed in terms of the operators (2.16). 
2.3. About homogenization. It is more convenient to start with the vector Pauli operator. As usual, we define the operators $\mathcal{P}_{\varepsilon}$ and $\mathcal{P}_{\varepsilon}^{0}$, replacing $f_{0}(\mathbf{x})$ by $f_{0}^{\varepsilon}(\mathbf{x})=f_{0}\left(\varepsilon^{-1} \mathbf{x}\right)$. Theorems $4.2 .3,4.2 .6,4.2 .9,4.4 .2$, and 4.4 .8 are applicable. Here it is convenient to change the notation somewhat. Let $\widetilde{\omega}_{ \pm}$be a positive function belonging to the kernel $\mathfrak{N}_{ \pm}$and such that $\left\|\widetilde{\omega}_{ \pm}\right\|_{L_{2}(\Omega)}^{2}=|\Omega|$. We put $\widetilde{f}=\operatorname{diag}\left\{\widetilde{\omega}_{-}, \widetilde{\omega}_{+}\right\}$. Let $\gamma$ be the number (2.17). Then, by Theorem 4.2.6,

$$
\left\|\left(\mathcal{P}_{\varepsilon}+I\right)^{-1}-\widetilde{f}^{\varepsilon}(-\gamma \Delta+I)^{-1} \widetilde{f}^{\varepsilon}\right\|_{\mathfrak{G} \rightarrow \mathfrak{G}} \leq C \varepsilon,
$$

and the constant $C$ can easily be decoded. Theorem 4.2.9 shows that the limit

$$
(w, \mathfrak{G} \rightarrow \mathfrak{G})-\lim _{\varepsilon \rightarrow 0}\left(\mathcal{P}_{\varepsilon}+I\right)^{-1}=\overline{(\widetilde{f})}(-\gamma \Delta+I)^{-1} \overline{(\widetilde{f})}
$$

exists.

Now, let $\widetilde{\mathbf{u}}_{\varepsilon}$ be the solution of the equation

$$
\mathcal{P}_{\varepsilon} \widetilde{\mathbf{u}}_{\varepsilon}+\widetilde{\mathbf{u}}_{\varepsilon}=\mathbf{F}, \quad \mathbf{F} \in \mathfrak{G},
$$

and let $\mathbf{u}_{0}$ be the solution of the equation

$$
-\gamma \Delta \mathbf{u}_{0}+\mathbf{u}_{0}=\operatorname{diag}\left\{\overline{\omega_{-}}\left(\overline{\omega_{-}^{2}}\right)^{-1}, \overline{\omega_{+}}\left(\overline{\omega_{+}^{2}}\right)^{-1}\right\} \mathbf{F} .
$$

Then, by Theorems 4.4.2 and 4.4.8 $\left(2^{\circ}\right)$ (with $\mathfrak{Q}=\mathbf{1}_{2}$ ), we have

$$
\begin{aligned}
\left(w, \mathfrak{G}^{1}\right)-\lim _{\varepsilon \rightarrow 0} f_{0}^{\varepsilon} \widetilde{\mathbf{u}}_{\varepsilon} & =\mathbf{u}_{0}, \\
(\mathfrak{G})-\lim _{\varepsilon \rightarrow 0} \widetilde{\mathbf{p}}_{\varepsilon} & =\mathbf{p}_{0} .
\end{aligned}
$$

Here the flows $\widetilde{\mathbf{p}}_{\varepsilon}, \mathbf{p}_{0}$ are defined by the formulas

$$
\begin{aligned}
\widetilde{\mathbf{p}}_{\varepsilon}=\left(f_{0}^{\varepsilon}\right)^{2} \mathcal{E} f_{0}^{\varepsilon} \widetilde{\mathbf{u}}_{\varepsilon} & =\left(\begin{array}{cc}
0 & \left(\omega_{+}^{\varepsilon}\right)^{2} \partial_{-} \omega_{-}^{\varepsilon} \\
\left(\omega_{-}^{\varepsilon}\right)^{2} \partial_{+} \omega_{+}^{\varepsilon} & 0
\end{array}\right) \widetilde{\mathbf{u}}_{\varepsilon}, \\
\mathbf{p}_{0} & =\left(\begin{array}{cc}
0 & \left(\overline{\omega_{-}^{2}}\right)^{-1} \partial_{-} \\
\left(\overline{\omega_{+}^{2}}\right)^{-1} \partial_{+} & 0
\end{array}\right) \mathbf{u}_{0} .
\end{aligned}
$$

The homogenization results for $\mathcal{P}$ directly imply the corresponding results for the blocks $P_{ \pm}$. In the spirit of (2.13), we put

$$
P_{+, \varepsilon}=Y_{\varepsilon}^{*} Y_{\varepsilon}, \quad P_{-, \varepsilon}=Y_{\varepsilon} Y_{\varepsilon}^{*}, \quad Y_{\varepsilon}=\omega_{+}^{\varepsilon} \partial_{-} \omega_{-}^{\varepsilon} .
$$

Then $\mathcal{P}_{\varepsilon}=\operatorname{diag}\left\{P_{-, \varepsilon}, P_{+, \varepsilon}\right\}$, and (2.18) yields

$$
\left\|\left(P_{ \pm, \varepsilon}+I\right)^{-1}-\widetilde{\omega}_{ \pm}^{\varepsilon}(-\gamma \Delta+I)^{-1} \widetilde{\omega}_{ \pm}^{\varepsilon}\right\|_{L_{2}\left(\mathbb{R}^{2}\right) \rightarrow L_{2}\left(\mathbb{R}^{2}\right)} \leq C \varepsilon .
$$

From (2.19) it follows that

$$
\begin{gathered}
\left(w, L_{2}\left(\mathbb{R}^{2}\right) \rightarrow L_{2}\left(\mathbb{R}^{2}\right)\right)-\lim _{\varepsilon \rightarrow 0}\left(P_{ \pm, \varepsilon}+I\right)^{-1}=\left(\overline{\widetilde{\omega}_{ \pm}}\right)^{2}(-\gamma \Delta+I)^{-1} \\
=\left(\overline{\omega_{ \pm}}\right)^{2}\left(\overline{\omega_{ \pm}^{2}}\right)^{-1}(-\gamma \Delta+I)^{-1} .
\end{gathered}
$$

Now, let $\widetilde{u}_{\varepsilon}^{( \pm)}$be the solution of the equation

$$
P_{ \pm, \varepsilon} \widetilde{u}_{\varepsilon}^{( \pm)}+\widetilde{u}_{\varepsilon}^{( \pm)}=F_{ \pm}, \quad F_{ \pm} \in L_{2}\left(\mathbb{R}^{2}\right),
$$

and let $u_{0}^{( \pm)}$be the solution of the equation

$$
-\gamma \Delta u_{0}^{( \pm)}+u_{0}^{( \pm)}=\overline{\omega_{ \pm}}\left(\overline{\omega_{ \pm}^{2}}\right)^{-1} F_{ \pm} .
$$


Then, by (2.20)-(2.25),

$$
\begin{aligned}
&\left(w, H^{1}\left(\mathbb{R}^{2}\right)\right)-\lim _{\varepsilon \rightarrow 0} \omega_{\mp}^{\varepsilon} \widetilde{u}_{\varepsilon}^{( \pm)}=u_{0}^{( \pm)}, \\
&\left(L_{2}\left(\mathbb{R}^{2}\right)\right)-\lim _{\varepsilon \rightarrow 0} \widetilde{p}_{\varepsilon}^{( \pm)}=p_{0}^{( \pm)},
\end{aligned}
$$

where the flows $\widetilde{p}_{\varepsilon}^{( \pm)}, p_{0}^{( \pm)}$are defined by the formulas

$$
\begin{aligned}
& \widetilde{p}_{\varepsilon}^{( \pm)}=\left(\omega_{ \pm}^{\varepsilon}\right)^{2} \partial_{\mp} \omega_{\mp}^{\varepsilon} \widetilde{u}_{\varepsilon}^{( \pm)}, \\
& p_{0}^{( \pm)}=\left(\overline{\omega_{\mp}^{2}}\right)^{-1} \partial_{\mp} u_{0}^{( \pm)} .
\end{aligned}
$$

2.4. The periodic Pauli operator with metric. Suppose that $\widetilde{g}(\mathbf{x})$ is a $\Gamma$-periodic $(2 \times 2)$-matrix-valued function with real entries and such that $\widetilde{g}(\mathbf{x})>0$ and $\widetilde{g}+\widetilde{g}^{-1} \in L_{\infty}$. In the space $L_{2}\left(\mathbb{R}^{2} ; \mathbb{C}^{2}\right)$, we consider the operator

$$
\mathcal{P}(g)=f_{0} \mathcal{E} g \mathcal{E} f_{0}, \quad g=f_{0} \widetilde{g} f_{0} .
$$

If $\widetilde{g}=\mathbf{1}_{2}$, the operator (2.32) turns into the Pauli operator $\mathcal{P}$. However, in contrast to $\mathcal{P}$, in the general case $\mathcal{P}(g)$ does not split into blocks. We note that

$$
\mathcal{P}(g)=f_{0} \widehat{\mathcal{B}}_{\times}(g) f_{0}, \quad g=f_{0} \widetilde{g} f_{0},
$$

where the operator $\widehat{\mathcal{B}}_{\times}(g)$ is defined by (5.1.22).

The operator $\mathcal{P}(g)$ falls into the general pattern with $m=n=d=2$ and $\mathfrak{G}=\mathfrak{G}_{*}=$ $L_{2}\left(\mathbb{R}^{2} ; \mathbb{C}^{2}\right)$. From $(2.33)$ it is clear that the operator $(2.32)$ can be written as $\mathcal{A}\left(g, f_{0}\right)$ with $b(\xi)=b_{\times}(\xi)$ defined by (5.1.20). Now the kernel $\mathfrak{N}$ has the form

$$
\mathfrak{N}=\left\{\mathbf{u} \in L_{2}\left(\Omega ; \mathbb{C}^{2}\right): f_{0} \mathbf{u}=\mathbf{c} \in \mathbb{C}^{2}\right\} .
$$

The effective matrix $g^{0}$ is unique and is defined by (5.1.23). The effective DO is given by the formula

$$
\mathcal{P}\left(g^{0}\right)=f_{0}(\mathbf{x}) \mathcal{E} g^{0} \mathcal{E} f_{0}(\mathbf{x}) .
$$

Now, the germ $S(\boldsymbol{\theta})$ (acting in $\mathfrak{N}$ ) of the operator $\mathcal{P}(g)$ depends on $\boldsymbol{\theta}$. However, it can be checked that its eigenvalues $\gamma_{1}(\boldsymbol{\theta})$ and $\gamma_{2}(\boldsymbol{\theta})$ coincide with the eigenvalues of the constant matrix

$$
|\Omega| f_{*} g^{0} f_{*}, \quad f_{*}:=\operatorname{diag}\left\{\left\|\omega_{+}\right\|_{L_{2}(\Omega)}^{-1},\left\|\omega_{-}\right\|_{L_{2}(\Omega)}^{-1}\right\} .
$$

Thus, the eigenvalues of the germ do not depend on $\theta$. The said above can be verified easily by using (1.1.22); see [BSu2, Subsection 7.3] for the details.

The homogenization problem for the operator $\mathcal{P}_{\varepsilon}(g)$ can be studied like this was done for the operator $\mathcal{P}_{\varepsilon}$ in Subsection 2.3. We shall not dwell on this.

\section{§3. Homogenization PRoblem for the operator $\mathcal{D}$}

3.1. Here we study the operator $\mathcal{D}$ introduced in Subsection 2.1. This operator will be analyzed by the method described in Subsection 1.1.8, though now there is no need to use the abstract results directly. Note that $\mathcal{D}$ may be interpreted as the two-dimensional Dirac operator with zero mass in the absence of electric field. However, for us, this operator is interesting as a model one. In the study of it, we clarify some features that are also typical for the much more difficult case of the Maxwell operator. The latter will be considered in Chapter 7 . 
3.2. We start with the definition (2.11):

$$
\mathcal{D}=f_{0} \mathcal{E} f_{0}=\left(\begin{array}{cc}
0 & Y \\
Y^{*} & 0
\end{array}\right)
$$

where the operator $Y$ is given by (2.13). As usual, we introduce the operator

$$
\mathcal{D}_{\varepsilon}=f_{0}^{\varepsilon} \mathcal{E} f_{0}^{\varepsilon}=\left(\begin{array}{cc}
0 & Y_{\varepsilon} \\
Y_{\varepsilon}^{*} & 0
\end{array}\right)=\left(\begin{array}{cc}
0 & \omega_{+}^{\varepsilon} \partial_{-} \omega_{-}^{\varepsilon} \\
\omega_{-}^{\varepsilon} \partial_{+} \omega_{+}^{\varepsilon} & 0
\end{array}\right), \quad \varepsilon>0 .
$$

Let $\mathbf{u}_{\varepsilon}=\operatorname{col}\left(u_{\varepsilon}^{(-)}, u_{\varepsilon}^{(+)}\right)$be the solution of the equation

$$
\left(\mathcal{D}_{\varepsilon}-i I\right) \mathbf{u}_{\varepsilon}=\mathbf{s}=\operatorname{col}(q, r), \quad \mathbf{s} \in \mathfrak{G}=L_{2}\left(\mathbb{R}^{2} ; \mathbb{C}^{2}\right) .
$$

We write $\mathbf{u}_{\varepsilon}$ as

$$
\mathbf{u}_{\varepsilon}=\mathbf{u}_{q, \varepsilon}+\mathbf{u}_{r, \varepsilon}, \quad \mathbf{u}_{q, \varepsilon}=\operatorname{col}\left(u_{q, \varepsilon}^{(-)}, u_{q, \varepsilon}^{(+)}\right), \quad \mathbf{u}_{r, \varepsilon}=\operatorname{col}\left(u_{r, \varepsilon}^{(-)}, u_{r, \varepsilon}^{(+)}\right),
$$

where $\mathbf{u}_{q, \varepsilon}$ is the solution of equation (3.2) with $r=0$, and $\mathbf{u}_{r, \varepsilon}$ is the solution of (3.2) with $q=0$.

Let $\mathbf{u}_{0}=\operatorname{col}\left(u_{0}^{(-)}, u_{0}^{(+)}\right)$be the solution of the equation

$$
\left(\mathcal{D}^{0}-i \Lambda\right) \mathbf{u}_{0}=\mathbf{s}=\operatorname{col}(q, r), \quad \mathbf{s} \in \mathfrak{G},
$$

where

$$
\mathcal{D}^{0}=\left(\underline{\omega_{-}}\right) \underline{\left(\omega_{+}\right)}\left(\begin{array}{cc}
0 & \partial_{-} \\
\partial_{+} & 0
\end{array}\right), \quad \Lambda=\left(\begin{array}{cc}
\left(\widetilde{\omega}_{-}\right)^{-2} & 0 \\
0 & \left(\widetilde{\omega}_{+}\right)^{-2}
\end{array}\right) .
$$

As in (3.3), we write $\mathbf{u}_{0}$ as

$$
\mathbf{u}_{0}=\mathbf{u}_{q, 0}+\mathbf{u}_{r, 0}, \quad \mathbf{u}_{q, 0}=\operatorname{col}\left(u_{q, 0}^{(-)}, u_{q, 0}^{(+)}\right), \quad \mathbf{u}_{r, 0}=\operatorname{col}\left(u_{r, 0}^{(-)}, u_{r, 0}^{(+)}\right) .
$$

Our goal is to prove the following theorem.

Theorem 3.1. $1^{\circ}$. Let $\mathcal{D}_{\varepsilon}$ be the operator defined by (3.1). Suppose that the operator $\mathcal{D}^{0}$ and the matrix $\Lambda$ are as in (3.5). Then

$$
(w, \mathfrak{G} \rightarrow \mathfrak{G})-\lim _{\varepsilon \rightarrow 0}\left(\mathcal{D}_{\varepsilon}-i I\right)^{-1}=\left(\mathcal{D}^{0}-i \Lambda\right)^{-1} .
$$

$2^{\circ}$. We have

$$
\begin{aligned}
\left(w, H^{1}\left(\mathbb{R}^{2}\right)\right)-\lim _{\varepsilon \rightarrow 0} \omega_{+}^{\varepsilon} u_{q, \varepsilon}^{(-)} & =\underline{\omega_{+}} u_{q, 0}^{(-)}, \\
\left(w, H^{1}\left(\mathbb{R}^{2}\right)\right)-\lim _{\varepsilon \rightarrow 0} \omega_{-}^{\varepsilon} u_{r, \varepsilon}^{(+)} & =\underline{\omega_{-}} u_{r, 0}^{(+)}, \\
\left(L_{2}\left(\mathbb{R}^{2}\right)\right)-\lim _{\varepsilon \rightarrow 0} \omega_{-}^{\varepsilon} u_{q, \varepsilon}^{(+)} & =\underline{\omega_{-}} u_{q, 0}^{(+)}, \\
\left(L_{2}\left(\mathbb{R}^{2}\right)\right)-\lim _{\varepsilon \rightarrow 0} \omega_{+}^{\varepsilon} u_{r, \varepsilon}^{(-)} & =\underline{\omega_{+}} u_{r, 0}^{(-)} .
\end{aligned}
$$

It is natural to call the operator $\mathcal{D}^{0}$ the effective $D O$ for $\mathcal{D}$. However, the right-hand side of (3.6) is a generalized resolvent. Relations (3.7)-(3.10) show that the results about the limit procedure are of different strength for different "blocks" of the solution $\mathbf{u}_{\varepsilon}$. The combination of these results leads inevitably to losses. However, we formulate the "united" result. Let $f_{0}$ be the matrix defined by (2.9). From (3.7)-(3.10) it is clear that $f_{0}^{\varepsilon} \mathbf{u}_{\varepsilon}$ is represented as the sum of two terms. One of them converges weakly in $\mathfrak{G}^{1}=H^{1}\left(\mathbb{R}^{2} ; \mathbb{C}^{2}\right)$, and the other converges strongly in $\mathfrak{G}$. Then the sum converges strongly in $\mathfrak{G}_{\text {loc }}$. As a result, we obtain the following statement.

Theorem 3.2. Under the assumptions of Theorem 3.1, we have

$$
\left(\mathfrak{G}_{\text {loc }}\right)-\lim _{\varepsilon \rightarrow 0} f_{0}^{\varepsilon} \mathbf{u}_{\varepsilon}=\underline{f_{0}} \mathbf{u}_{0} .
$$


3.3. Proof of Theorem 3.1. The summands $\mathbf{u}_{q, \varepsilon}$ and $\mathbf{u}_{r, \varepsilon}$ in (3.3) are treated similarly. For definiteness, we consider $\mathbf{u}_{q, \varepsilon}$. From equation (3.2) with $r=0$, we have

$$
Y_{\varepsilon} u_{q, \varepsilon}^{(+)}-i u_{q, \varepsilon}^{(-)}=q, \quad Y_{\varepsilon}^{*} u_{q, \varepsilon}^{(-)}-i u_{q, \varepsilon}^{(+)}=0 .
$$

From (3.11) it follows that

$$
\begin{aligned}
u_{q, \varepsilon}^{(-)}=i\left(Y_{\varepsilon} Y_{\varepsilon}^{*}+I\right)^{-1} q & =i\left(P_{-, \varepsilon}+I\right)^{-1} q, \\
u_{q, \varepsilon}^{(+)} & =Y_{\varepsilon}^{*}\left(P_{-, \varepsilon}+I\right)^{-1} q,
\end{aligned}
$$

where $P_{-, \varepsilon}$ is defined by (2.26). Relations (3.12) and (2.28-) show that

$$
\left(w, L_{2}\left(\mathbb{R}^{2}\right)\right)-\lim _{\varepsilon \rightarrow 0} u_{q, \varepsilon}^{(-)}=i\left(\widetilde{\widetilde{\omega}}_{-}\right)^{2}(-\gamma \Delta+I)^{-1} q .
$$

By (3.12) and (2.29-) (with $F_{-}=i q$ ), we obtain

$$
\left(w, H^{1}\left(\mathbb{R}^{2}\right)\right)-\lim _{\varepsilon \rightarrow 0} \omega_{+}^{\varepsilon} u_{q, \varepsilon}^{(-)}=i \overline{\omega_{-}}\left(\overline{\omega_{-}^{2}}\right)^{-1}(-\gamma \Delta+I)^{-1} q .
$$

Combining (3.13), (2.26), and (2.31-) (with $F_{-}=q$ ), we see that

$$
u_{q, \varepsilon}^{(+)}=\omega_{+}^{\varepsilon} \widetilde{p}_{\varepsilon}^{(-)}, \quad \widetilde{p}_{\varepsilon}^{(-)}=\left(\omega_{-}^{\varepsilon}\right)^{2} \partial_{+} \omega_{+}^{\varepsilon}\left(P_{-, \varepsilon}+I\right)^{-1} q .
$$

This and (2.30-) imply the strong $L_{2}\left(\mathbb{R}^{2}\right)$-convergence for the product $\omega_{-}^{\varepsilon} u_{q, \varepsilon}^{(+)}$:

$$
\left(L_{2}\left(\mathbb{R}^{2}\right)\right)-\lim _{\varepsilon \rightarrow 0} \omega_{-}^{\varepsilon} u_{q, \varepsilon}^{(+)}=p_{0}^{(-)}=\overline{\omega_{-}} \gamma \partial_{+}(-\gamma \Delta+I)^{-1} q .
$$

By Proposition 4.0.1, relation (3.16) implies

$$
\left(w, L_{2}\left(\mathbb{R}^{2}\right)\right)-\lim _{\varepsilon \rightarrow 0} u_{q, \varepsilon}^{(+)}=\left(\overline{\omega_{-}}\right)\left(\overline{\omega_{+}}\right) \gamma \partial_{+}(-\gamma \Delta+I)^{-1} q .
$$

Similarly, using the properties of the operator $P_{+, \varepsilon}$, we arrive at the following convergence results for $u_{r, \varepsilon}^{(-)}, u_{r, \varepsilon}^{(+)}$:

$$
\begin{aligned}
&\left(w, L_{2}\left(\mathbb{R}^{2}\right)\right)-\lim _{\varepsilon \rightarrow 0} u_{r, \varepsilon}^{(+)}=i\left(\overline{\widetilde{\omega}_{+}}\right)^{2}(-\gamma \Delta+I)^{-1} r, \\
&\left(w, H^{1}\left(\mathbb{R}^{2}\right)\right)-\lim _{\varepsilon \rightarrow 0} \omega_{-}^{\varepsilon} u_{r, \varepsilon}^{(+)}=i \overline{\omega_{+}}\left(\overline{\omega_{+}^{2}}\right)^{-1}(-\gamma \Delta+I)^{-1} r, \\
&\left(L_{2}\left(\mathbb{R}^{2}\right)\right)-\lim _{\varepsilon \rightarrow 0} \omega_{+}^{\varepsilon} u_{r, \varepsilon}^{(-)}=\overline{\omega_{+}} \gamma \partial_{-}(-\gamma \Delta+I)^{-1} r, \\
&\left(w, L_{2}\left(\mathbb{R}^{2}\right)\right)-\lim _{\varepsilon \rightarrow 0} u_{r, \varepsilon}^{(-)}=\left(\overline{\omega_{-}}\right)\left(\overline{\omega_{+}}\right) \gamma \partial_{-}(-\gamma \Delta+I)^{-1} r .
\end{aligned}
$$

Finally, relations (3.3), (3.14), (3.17), (3.18), and (3.21) show that

$$
(w, \mathfrak{G})-\lim _{\varepsilon \rightarrow 0} \mathbf{u}_{\varepsilon}=\mathbf{u}_{0}=\operatorname{col}\left(u_{0}^{(-)}, u_{0}^{(+)}\right),
$$

where

$$
\begin{aligned}
& u_{0}^{(-)}=i\left(\overline{\widetilde{\omega}_{-}}\right)^{2}(-\gamma \Delta+I)^{-1} q+\left(\overline{\omega_{-}}\right)\left(\overline{\omega_{+}}\right) \gamma \partial_{-}(-\gamma \Delta+I)^{-1} r, \\
& u_{0}^{(+)}=i\left(\overline{\widetilde{\omega}_{+}}\right)^{2}(-\gamma \Delta+I)^{-1} r+\left(\overline{\omega_{-}}\right)\left(\overline{\omega_{+}}\right) \gamma \partial_{+}(-\gamma \Delta+I)^{-1} q .
\end{aligned}
$$

It can be checked directly that $\mathbf{u}_{0}$ satisfies equation (3.4). Now, (3.22) implies (3.6).

Relations (3.7), (3.8), (3.9), and (3.10) follow from (3.15), (3.19), (3.16), and (3.20), respectively.

We note that, for $u_{q, \varepsilon}^{(-)}$and $u_{r, \varepsilon}^{(+)}$, some results can be deduced from $(2.27 \pm)$. We write the estimate for $u_{q, \varepsilon}^{(-)}$:

$$
\left\|u_{q, \varepsilon}^{(-)}-i \widetilde{\omega}_{-}^{\varepsilon}(-\gamma \Delta+I)^{-1} \widetilde{\omega}_{-}^{\varepsilon} q\right\|_{L_{2}\left(\mathbb{R}^{2}\right)} \leq C \varepsilon\|q\|_{L_{2}\left(\mathbb{R}^{2}\right)} .
$$

Homogenization of the oscillating factors $\widetilde{\omega}_{-}^{\varepsilon}$ necessarily affects the quality of convergence (cf. (3.14)). 


\section{Comments On Chapter 6}

1. About factorization for the Schrödinger operator, see, e.g., [KiSi, BSu1]. Apparently, Proposition 1.3 (formula (1.12)) was mentioned in [BSu1] for the first time. The results of $\S 1$ about homogenization are new; at least, this concerns Theorems 1.5-1.7. Especially, we distinguish estimate (1.16).

2. The two-dimensional periodic Pauli operator was treated by the authors in BSu1 BSu2. In these papers the factorization formulas were given, and it was shown that the effective masses for the two-dimensional Pauli operator can be calculated explicitly and do not depend on the direction of the quasimomentum, i.e., on the vector $\boldsymbol{\theta}$. All the results concerning homogenization for the Pauli operator are new. In this case, we benefited from the relation $m=n$.

3. For the first time, the Pauli operator with metric was introduced in [BSu1], although the version suggested in that paper does not seem appropriate. The version considered in Subsection 2.4 was proposed in [BSu2]. From the mathematical point of view, its definition looks natural, although the authors cannot suggest a physical interpretation for this operator.

4. The operator $\mathcal{D}$ from $\S 3$ is discussed by way of illustration. It is instructive that, even for proving the existence of the weak limit (3.6), we had to use the specific properties of the case where $m=n$.

\section{Chapter 7. The Periodic Maxwell operator}

\section{$\S 1$. PRELIMINARY REMARKS}

In this chapter, we apply the general results of Chapters 3 and 4 to the stationary periodic Maxwell operator, one of the cases that are most difficult and important for applications. Mainly, we concentrate on homogenization problems. The study of the Maxwell operator encounters a number of complications. First, written in terms of electric and magnetic field strengths, the Maxwell operator acts in weighted $L_{2}$-spaces, which depend on the parameter $\varepsilon$. Therefore, the question about the resolvent convergence loses its direct meaning. Second, the Maxwell operator has "reasonable" properties only in the corresponding solenoidal spaces, which, moreover, depend on $\varepsilon$. In order to avoid these difficulties, we write the Maxwell operator in terms of displacements rather than strengths. Thereby, we are forced to sacrifice selfadjointness. Some results for strengths can be obtained afterwards.

Next, it turns out that in the study of homogenization for the Maxwell operator it is convenient to represent each field as a sum of two terms, like it was done in Subsection 1.1.8 and also in $\S 6.3$ (for the operator $\mathcal{D}$ ). In this way, the results for different terms are of different quality.

We cannot include the general Maxwell operator in the scheme developed above; we have to assume that one of the two coefficients (dielectric permittivity $\epsilon$ or magnetic permeability $\mu$ ) is unit. This assumption is caused by the fact that only in this case can the study be based on the results about homogenization for an appropriate operator of the form $\widehat{\mathcal{A}}(\mathrm{g})$. We give a preliminary treatment of such an operator in $\S 2$. Results about homogenization for the Maxwell operator are presented in $\S 3$. In the Comments on Chapter 7 , we compare the results obtained here with the known ones. For definiteness, we assume that $\mu=1$; this is preferable from the physical point of view.

The second author is going to devote a separate paper to the study of the homogenization problem for the periodic Maxwell operator in the general case. 


\section{$\S 2$. The operator $\mathcal{L}(\epsilon, \nu)=\operatorname{rot} \epsilon^{-1} \operatorname{rot}-\nabla \nu \operatorname{div}$}

2.1. Definition of the operator. Let $\epsilon(\mathbf{x})$ be a $\Gamma$-periodic $(3 \times 3)$-matrix-valued function in $\mathbb{R}^{3}$ with real entries and such that

$$
\epsilon(\mathbf{x})>0, \quad \epsilon+\epsilon^{-1} \in L_{\infty} .
$$

Let $\nu(\mathbf{x})$ be a real-valued $\Gamma$-periodic function in $\mathbb{R}^{3}$ such that

$$
\nu(\mathbf{x})>0, \quad \nu+\nu^{-1} \in L_{\infty} .
$$

In $\mathfrak{G}=L_{2}\left(\mathbb{R}^{3} ; \mathbb{C}^{3}\right)$, we consider the operator $\mathcal{L}(\epsilon, \nu)$ given formally by the expression

$$
\mathcal{L}(\epsilon, \nu)=\operatorname{rot}(\epsilon(\mathbf{x}))^{-1} \operatorname{rot}-\nabla \nu(\mathbf{x}) \operatorname{div} .
$$

The precise definition of $\mathcal{L}(\epsilon, \nu)$ as a selfadjoint operator in $\mathfrak{G}$ is given via the closed positive form

$$
\int_{\mathbb{R}^{3}}\left(\left\langle(\epsilon(\mathbf{x}))^{-1} \operatorname{rot} \mathbf{u}, \operatorname{rot} \mathbf{u}\right\rangle+\nu(\mathbf{x})|\operatorname{div} \mathbf{u}|^{2}\right) d \mathbf{x}, \quad \mathbf{u} \in \mathfrak{G}^{1}=H^{1}\left(\mathbb{R}^{3} ; \mathbb{C}^{3}\right) .
$$

The operator $\mathcal{L}(\epsilon, \nu)$ is of the form $\widehat{\mathcal{A}}(g)=b(\mathbf{D})^{*} g(\mathbf{x}) b(\mathbf{D})$ with $n=3, m=4, \mathfrak{G}_{*}=$ $L_{2}\left(\mathbb{R}^{3} ; \mathbb{C}^{4}\right)$,

$$
b(\mathbf{D})=\left(\begin{array}{l}
-i \operatorname{rot} \\
-i \operatorname{div}
\end{array}\right), \quad g(\mathbf{x})=\left(\begin{array}{cc}
(\epsilon(\mathbf{x}))^{-1} & 0 \\
0 & \nu(\mathbf{x})
\end{array}\right) .
$$

The corresponding symbol $b(\xi)$ looks like this:

$$
b(\xi)=\left(\begin{array}{ccc}
0 & -\xi^{3} & \xi^{2} \\
\xi^{3} & 0 & -\xi^{1} \\
-\xi^{2} & \xi^{1} & 0 \\
\xi^{1} & \xi^{2} & \xi^{3}
\end{array}\right) .
$$

Obviously, $\operatorname{rank} b(\xi)=3$ for $\xi \neq 0$, i.e., condition $(2.1 .2)$ is satisfied. Now $\mathfrak{H}=L_{2}\left(\Omega ; \mathbb{C}^{3}\right)$. The kernel $\widehat{\mathfrak{N}}$ is defined in accordance with (3.1.1):

$$
\widehat{\mathfrak{N}}=\left\{\mathbf{u} \in \mathfrak{H}: \mathbf{u}=\mathbf{c} \in \mathbb{C}^{3}\right\} .
$$

2.2. The effective matrix $g^{0}$ for the operator $\mathcal{L}(\epsilon, \nu)$ can be calculated by the general rules described in $\S 3.1$. Let $\mathbf{C} \in \mathbb{C}^{4}$, and let $\mathbf{v} \in \widetilde{H}^{1}\left(\Omega ; \mathbb{C}^{3}\right)$ be the solution of the equation

$$
b(\mathbf{D})^{*} g(\mathbf{x})(b(\mathbf{D}) \mathbf{v}+\mathbf{C})=0,
$$

which now takes the form

$$
\operatorname{rot} \epsilon(\mathbf{x})^{-1}(\operatorname{rot} \mathbf{v}+i \widetilde{\mathbf{C}})-\nabla \nu(\mathbf{x})\left(\operatorname{div} \mathbf{v}+i C^{4}\right)=0,
$$

where $\mathbf{C}=\sum_{j=1}^{4} C^{j} \mathbf{e}_{j}, \widetilde{\mathbf{C}}=\sum_{j=1}^{3} C^{j} \mathbf{e}_{j}$. In other words, $\mathbf{v} \in \widetilde{H}^{1}\left(\Omega ; \mathbb{C}^{3}\right)$ satisfies an identity of the form (3.1.3):

$$
\begin{array}{r}
\int_{\Omega}\left(\left\langle\epsilon(\mathbf{x})^{-1}(\operatorname{rot} \mathbf{v}+i \widetilde{\mathbf{C}}), \operatorname{rot} \mathbf{z}\right\rangle_{\mathbb{C}^{3}}+\nu(\mathbf{x})\left(\operatorname{div} \mathbf{v}+i C^{4}\right)(\operatorname{div} \mathbf{z})^{+}\right) d \mathbf{x}=0, \\
\mathbf{z} \in \widetilde{H}^{1}\left(\Omega ; \mathbb{C}^{3}\right) .
\end{array}
$$

Representing $\mathbf{z}$ as the sum $\mathbf{z}=\widetilde{\mathbf{z}}+\nabla \varphi$, where $\operatorname{div} \widetilde{\mathbf{z}}=0$, we write (2.5) for $\mathbf{z}=\widetilde{\mathbf{z}}$. The term with $\operatorname{div} \widetilde{\mathbf{z}}$ will disappear. By the identity $\operatorname{rot} \widetilde{\mathbf{z}}=\operatorname{rot} \mathbf{z}$, we obtain

$$
\int_{\Omega}\left\langle\epsilon(\mathbf{x})^{-1}(\operatorname{rot} \mathbf{v}+i \widetilde{\mathbf{C}}), \operatorname{rot} \mathbf{z}\right\rangle_{\mathbb{C}^{3}} d \mathbf{x}=0, \quad \mathbf{z} \in \widetilde{H}^{1}\left(\Omega ; \mathbb{C}^{3}\right) .
$$

From (2.6) it follows that

$$
\epsilon(\mathbf{x})^{-1}(\operatorname{rot} \mathbf{v}+i \widetilde{\mathbf{C}})=\nabla \Phi+i \mathbf{c}, \Phi \in \widetilde{H}^{1}(\Omega), \quad \mathbf{c} \in \mathbb{C}^{3},
$$


whence

$$
\operatorname{rot} \mathbf{v}+i \widetilde{\mathbf{C}}=\epsilon(\mathbf{x})(\nabla \Phi+i \mathbf{c})
$$

Relation (2.8) implies

$$
\int_{\Omega}\langle\epsilon(\mathbf{x})(\nabla \Phi+i \mathbf{c}), \nabla \Psi\rangle_{\mathbb{C}^{3}} d \mathbf{x}=0, \quad \Psi \in \widetilde{H}^{1}(\Omega) .
$$

Thus, $\Phi$ is an $\widetilde{H}^{1}(\Omega)$-solution of the equation

$$
\operatorname{div} \epsilon(\mathbf{x})(\nabla \Phi+i \mathbf{c})=0, \quad \mathbf{c} \in \mathbb{C}^{3} .
$$

Let $\epsilon^{0}$ be the effective matrix for the acoustic operator $-\operatorname{div} \epsilon(\mathbf{x}) \nabla$. In accordance with the representation (3.1.14), we have

$$
\epsilon^{0} \mathbf{c}=|\Omega|^{-1} \int_{\Omega} \epsilon(\mathbf{x})(-i \nabla \Phi+\mathbf{c}) d \mathbf{x}, \quad \mathbf{c} \in \mathbb{C}^{3} .
$$

Integrating (2.8) and using (2.9), we obtain

$$
\widetilde{\mathbf{C}}=\epsilon^{0} \mathbf{c} .
$$

On the other hand, integrating (2.7), we arrive at the relation

$$
i \mathbf{c}|\Omega|=\int_{\Omega} \epsilon(\mathbf{x})^{-1}(\operatorname{rot} \mathbf{v}+i \widetilde{\mathbf{C}}) d \mathbf{x} .
$$

Next, (2.5) and (2.6) imply the identity

$$
\int_{\Omega} \nu(\mathbf{x})\left(\operatorname{div} \mathbf{v}+i C^{4}\right)(\operatorname{div} \mathbf{z})^{+} d \mathbf{x}=0, \quad \mathbf{z} \in \widetilde{H}^{1}\left(\Omega ; \mathbb{C}^{3}\right),
$$

which means that

$$
\nu(\mathbf{x})\left(\operatorname{div} \mathbf{v}+i C^{4}\right)=\alpha \in \mathbb{C} .
$$

From (2.12) it is clear that

$$
i C^{4}|\Omega|=\alpha \int_{\Omega} \nu(\mathbf{x})^{-1} d \mathbf{x},
$$

i.e.,

$$
\alpha=i C^{4} \underline{\nu} .
$$

Now we calculate $g^{0}$ starting with the representation (3.1.14) for the operator (2.2). By (2.10)-(2.13), we have

$$
\begin{aligned}
g^{0} \mathbf{C} & =|\Omega|^{-1} \int_{\Omega} g(\mathbf{x})(b(\mathbf{D}) \mathbf{v}+\mathbf{C}) d \mathbf{x}=|\Omega|^{-1} \int_{\Omega}\left(\begin{array}{c}
\epsilon(\mathbf{x})^{-1}(-i \operatorname{rot} \mathbf{v}+\widetilde{\mathbf{C}}) \\
\nu(\mathbf{x})\left(-i \operatorname{div} \mathbf{v}+C^{4}\right)
\end{array}\right) d \mathbf{x} \\
& =\left(\begin{array}{c}
\mathbf{c} \\
-i \alpha
\end{array}\right)=\left(\begin{array}{c}
\left(\epsilon^{0}\right)^{-1} \widetilde{\mathbf{C}} \\
\underline{\nu} C^{4}
\end{array}\right)=\left(\begin{array}{cc}
\left(\epsilon^{0}\right)^{-1} & 0 \\
m y O & \underline{\nu}
\end{array}\right) \mathbf{C} .
\end{aligned}
$$

Thus, the effective matrix $g^{0}$ of the operator $\mathcal{L}(\epsilon, \nu)$ can be expressed in terms of $\underline{\nu}$ and the effective matrix $\epsilon^{0}$ for the operator $-\operatorname{div} \epsilon(\mathbf{x}) \nabla$ :

$$
g^{0}=\operatorname{diag}\left\{\left(\epsilon^{0}\right)^{-1}, \underline{\nu}\right\} .
$$

It is easy to check that for the effective matrix with real entries the uniqueness condition (3.1.16) is satisfied. Indeed,

$$
b(\boldsymbol{\theta}) \mathbf{y}=\operatorname{col}(\boldsymbol{\theta} \times \mathbf{y}, \boldsymbol{\theta} \cdot \mathbf{y}), \quad \mathbf{y} \in \mathbb{R}^{3},
$$


whence

$$
\begin{aligned}
b(\boldsymbol{\theta}) \mathbb{R}^{3} & =\left\{\operatorname{col}(\mathbf{e}, a) \in \mathbb{R}^{4}: \mathbf{e} \perp \boldsymbol{\theta}, a \in \mathbb{R}\right\}, \\
\operatorname{clos} \bigcup_{\boldsymbol{\theta}} b(\boldsymbol{\theta}) \mathbb{R}^{3} & =\mathbb{R}^{4} .
\end{aligned}
$$

The following operator is the effective $D O$ for the operator $\mathcal{L}(\epsilon, \nu)$ :

$$
\mathcal{L}^{0}=\mathcal{L}\left(\epsilon^{0}, \underline{\nu}\right)=\operatorname{rot}\left(\epsilon^{0}\right)^{-1} \operatorname{rot}-\nabla \underline{\nu} \operatorname{div} .
$$

The germ $S_{\mathcal{L}}(\boldsymbol{\theta})$ corresponding to the operator $\mathcal{L}(\epsilon, \nu)$ acts in $\widehat{\mathfrak{N}}$ as multiplication by the matrix

$$
S_{\mathcal{L}}(\boldsymbol{\theta})=b(\boldsymbol{\theta})^{*} g^{0} b(\boldsymbol{\theta}), \quad \boldsymbol{\theta} \in S S^{2} .
$$

By (2.3) and (2.14), the germ $S_{\mathcal{L}}(\boldsymbol{\theta})$ can be represented as the sum

$$
S_{\mathcal{L}}(\boldsymbol{\theta})=b_{r}(\boldsymbol{\theta})^{*}\left(\epsilon^{0}\right)^{-1} b_{r}(\boldsymbol{\theta})+\underline{\nu} b_{d}(\boldsymbol{\theta})^{*} b_{d}(\boldsymbol{\theta}),
$$

where

$$
b_{r}(\boldsymbol{\theta})=\left(\begin{array}{ccc}
0 & -\theta^{3} & \theta^{2} \\
\theta^{3} & 0 & -\theta^{1} \\
-\theta^{2} & \theta^{1} & 0
\end{array}\right), \quad b_{d}(\boldsymbol{\theta})=\left(\begin{array}{lll}
\theta^{1} & \theta^{2} & \theta^{3}
\end{array}\right) .
$$

2.3. Splitting of the operator $\mathcal{L}(\epsilon, \nu)$. We use the Weyl decomposition

$$
\begin{aligned}
\mathfrak{G}=L_{2}\left(\mathbb{R}^{3} ; \mathbb{C}^{3}\right) & =J\left(\mathbb{R}^{3}\right) \oplus G\left(\mathbb{R}^{3}\right), \\
J\left(\mathbb{R}^{3}\right)=J & =\{\mathbf{w} \in \mathfrak{G}: \operatorname{div} \mathbf{w}=0\}, \\
G\left(\mathbb{R}^{3}\right) & =\left\{\mathbf{u}=\nabla \varphi: \varphi \in H_{\mathrm{loc}}^{1}\left(\mathbb{R}^{3}\right), \nabla \varphi \in \mathfrak{G}\right\} .
\end{aligned}
$$

Decomposition (2.18) reduces the operator $\mathcal{L}(\epsilon, \nu)$ :

$$
\mathcal{L}(\epsilon, \nu)=\mathcal{L}_{J}(\epsilon) \oplus \mathcal{L}_{G}(\nu) .
$$

The operator $\mathcal{L}_{J}(\epsilon)$ acting in the subspace $J\left(\mathbb{R}^{3}\right)$ corresponds to the differential expression $\operatorname{rot} \epsilon(\mathbf{x})^{-1}$ rot, and the operator $\mathcal{L}_{G}(\nu)$ acting in $G\left(\mathbb{R}^{3}\right)$ is defined by the expression $-\nabla \nu(\mathbf{x})$ div. We are interested mainly in the operator $\mathcal{L}_{J}(\epsilon)$, the definition of which does not depend on $\nu$.

Observe that the germ (2.16), which acts in the kernel (2.4), also splits in the "induced" orthogonal decomposition

$$
\begin{aligned}
\widehat{\mathfrak{N}} & =J(\boldsymbol{\theta}) \oplus G(\boldsymbol{\theta}), \\
J(\boldsymbol{\theta}) & =\left\{\mathbf{c} \in \mathbb{C}^{3}: \mathbf{c} \perp \boldsymbol{\theta}\right\}, \\
G(\boldsymbol{\theta}) & =\{\mathbf{c}=\lambda \boldsymbol{\theta}: \lambda \in \mathbb{C}\} .
\end{aligned}
$$

The part of $S_{\mathcal{L}}(\boldsymbol{\theta})$ in $J(\boldsymbol{\theta})$ is denoted by $S_{\mathcal{L}, J}(\boldsymbol{\theta})$ and corresponds to the first summand in (2.16), and the part of $S_{\mathcal{L}}(\boldsymbol{\theta})$ in $G(\boldsymbol{\theta})$ is denoted by $S_{\mathcal{L}, G}(\boldsymbol{\theta})$ and corresponds to the second summand. In the subspace $G(\boldsymbol{\theta})$, the operator $S_{\mathcal{L}}(\boldsymbol{\theta})$ has a unique eigenvalue $\gamma_{3}(\boldsymbol{\theta})=\underline{\nu}$. In the subspace $J(\boldsymbol{\theta})$, it has two eigenvalues $\gamma_{1}(\boldsymbol{\theta})$ and $\gamma_{2}(\boldsymbol{\theta})$, which correspond to the algebraic problem

$$
b_{r}(\boldsymbol{\theta})^{*}\left(\epsilon^{0}\right)^{-1} b_{r}(\boldsymbol{\theta}) \mathbf{c}=\gamma \mathbf{c}, \quad \mathbf{c} \perp \boldsymbol{\theta} .
$$

In some sense, $S_{\mathcal{L}, J}(\boldsymbol{\theta})$ plays the role of the germ for the operator $\mathcal{L}_{J}(\epsilon)$, and $S_{\mathcal{L}, G}(\boldsymbol{\theta})$ plays the role of the germ for $\mathcal{L}_{G}(\nu)$. We shall not dwell on the corresponding analysis. 
2.4. Homogenization. To the operator

$$
\mathcal{L}_{\varepsilon}(\epsilon, \nu)=\mathcal{L}\left(\epsilon^{\varepsilon}, \nu^{\varepsilon}\right)=\operatorname{rot}\left(\epsilon^{\varepsilon}\right)^{-1} \operatorname{rot}-\nabla \nu^{\varepsilon} \operatorname{div},
$$

we can apply the general theorems about homogenization for the case where $f=\mathbf{1}_{n}$. As in $\S 5.1$, we shall not write down the constants in estimates explicitly, though it is easy to do this. In what follows, we denote such constants by the letter $C$ without indices.

First, we apply Theorem 4.2.1. We use the fact that decomposition (2.18) simultaneously reduces the operator $\mathcal{L}_{\varepsilon}(\epsilon, \nu)$ and the effective operator $\mathcal{L}^{0}$ defined by $(2.15)$. Therefore, the estimate for the difference of the resolvents of $\mathcal{L}_{\varepsilon}(\epsilon, \nu)$ and $\mathcal{L}^{0}$ directly implies an estimate for the difference of the resolvents of the operators $\mathcal{L}_{J, \varepsilon}(\epsilon)=\mathcal{L}_{J}\left(\epsilon^{\varepsilon}\right)$ and $\mathcal{L}_{J}^{0}=\mathcal{L}_{J}\left(\epsilon^{0}\right)$, which act in the subspace $J$. As a result, we obtain the following statement.

Theorem 2.1. We have

$$
\begin{aligned}
\left\|\left(\mathcal{L}_{\varepsilon}(\epsilon, \nu)+I\right)^{-1}-\left(\mathcal{L}^{0}+I\right)^{-1}\right\|_{\mathfrak{G} \rightarrow \mathfrak{G}} \leq C \varepsilon, & 0<\varepsilon \leq 1 \\
\left\|\left(\mathcal{L}_{J, \varepsilon}(\epsilon)+I\right)^{-1}-\left(\mathcal{L}_{J}^{0}+I\right)^{-1}\right\|_{J \rightarrow J} \leq C \varepsilon, & 0<\varepsilon \leq 1
\end{aligned}
$$

Now we discuss application of the "interpolation" Theorem 4.2.7 (with $Q=I$ ). Let $P_{J}$ be the orthogonal projection of $\mathfrak{G}$ onto the subspace $J$. In the Fourier representation, the operator $P_{J}$ turns into multiplication by the symbol $\left(i b_{r}(\boldsymbol{\theta})\right)^{2}, \boldsymbol{\theta}=\boldsymbol{\xi} /|\xi|$, where $b_{r}$ is defined by (2.17). In other words, $P_{J}$ is a pseudodifferential operator of zero order, which acts continuously in each of the spaces $\mathfrak{G}^{s}=H^{s}\left(\mathbb{R}^{3} ; \mathbb{C}^{3}\right), s \in \mathbb{R}$. Moreover, let $P_{J}^{(s)}$ be the restriction of $P_{J}$ to $\mathfrak{G}^{s}$ for $s>0$ and the extension of $P_{J}$ by continuity to $\mathfrak{G}^{s}$ for $s<0$. Then $P_{J}^{(s)}$ is an orthogonal projection in all spaces $\mathfrak{G}^{s}, s \in \mathbb{R}$. We introduce the spaces $J^{s}=P_{J}^{(s)} \mathfrak{G}^{s}$ with the norm induced by the norm in $\mathfrak{G}^{s}$. The following result is true.

Theorem 2.2. For $0 \leq s<1$, we have

$$
\begin{aligned}
\left\|\left(\mathcal{L}_{\varepsilon}(\epsilon, \nu)+I\right)^{-1}-\left(\mathcal{L}^{0}+I\right)^{-1}\right\|_{\mathfrak{G}^{-s} \rightarrow \mathfrak{G}^{s}} \leq C \varepsilon^{1-s}, & 0<\varepsilon \leq 1, \\
\left\|\left(\mathcal{L}_{J, \varepsilon}(\epsilon)+I\right)^{-1}-\left(\mathcal{L}_{J}^{0}+I\right)^{-1}\right\|_{J^{-s} \rightarrow J^{s}} \leq C \varepsilon^{1-s}, & 0<\varepsilon \leq 1 .
\end{aligned}
$$

Here (2.19) is a direct consequence of Theorem 4.2.7, and (2.20) follows from (2.19), by applying the corresponding orthogonal projections.

Now we use Theorem 4.4.1 (again with $Q=I$ ). Let $\mathbf{v}_{\varepsilon}$ be the solution of the equation

$$
\mathcal{L}_{\varepsilon}(\epsilon, \nu) \mathbf{v}_{\varepsilon}+\mathbf{v}_{\varepsilon}=\mathbf{F}, \quad \mathbf{F} \in \mathfrak{G}^{-1},
$$

and let $\mathbf{v}_{0}$ be the solution of the equation

$$
\mathcal{L}^{0} \mathbf{v}_{0}+\mathbf{v}_{0}=\mathbf{F}, \quad \mathbf{F} \in \mathfrak{G}^{-1} .
$$

Observe that if $\mathbf{F} \in J^{-1}$, we automatically have $\operatorname{div} \mathbf{v}_{\varepsilon}=0$ and $\operatorname{div} \mathbf{v}_{0}=0$. In this case $\mathbf{v}_{\varepsilon}$ coincides with the solution of the problem

$$
\operatorname{rot}\left(\epsilon^{\varepsilon}(\mathbf{x})\right)^{-1} \operatorname{rot} \mathbf{v}_{\varepsilon}+\mathbf{v}_{\varepsilon}=\mathbf{F}, \quad \operatorname{div} \mathbf{v}_{\varepsilon}=0, \quad \mathbf{F} \in J^{-1},
$$

and $\mathbf{v}_{0}$ coincides with the solution of the problem

$$
\operatorname{rot}\left(\epsilon^{0}\right)^{-1} \operatorname{rot} \mathbf{v}_{0}+\mathbf{v}_{0}=\mathbf{F}, \quad \operatorname{div} \mathbf{v}_{0}=0, \quad \mathbf{F} \in J^{-1} .
$$

Theorem 4.4.1 implies the following result. 
Theorem 2.3. $1^{\circ}$. Let $\mathbf{v}_{\varepsilon}$ be the solution of equation (2.21), and $\mathbf{v}_{0}$ the solution of (2.22). Then

$$
\begin{aligned}
\left(w, \mathfrak{G}^{1}\right)-\lim _{\varepsilon \rightarrow 0} \mathbf{v}_{\varepsilon} & =\mathbf{v}_{0}, \\
\left(w, \mathfrak{G}_{*}\right)-\lim _{\varepsilon \rightarrow 0} g^{\varepsilon} b(\mathbf{D}) \mathbf{v}_{\varepsilon} & =g^{0} b(\mathbf{D}) \mathbf{v}_{0} .
\end{aligned}
$$

$2^{\circ}$. Let $\mathbf{v}_{\varepsilon}$ be the solution of problem (2.23), and $\mathbf{v}_{0}$ the solution of problem (2.24). Then

$$
\begin{aligned}
\left(w, J^{1}\right)-\lim _{\varepsilon \rightarrow 0} \mathbf{v}_{\varepsilon} & =\mathbf{v}_{0}, \\
(w, \mathfrak{G})-\lim _{\varepsilon \rightarrow 0}\left(\epsilon^{\varepsilon}\right)^{-1} \operatorname{rot} \mathbf{v}_{\varepsilon} & =\left(\epsilon^{0}\right)^{-1} \operatorname{rot} \mathbf{v}_{0} .
\end{aligned}
$$

We distinguish the case where the convergence of solutions or flows is strong. By (2.14), the case where $g^{0}=\bar{g}$ corresponds to $\epsilon^{0}=\underline{\epsilon}, \nu=$ const. The case where $g^{0}=g$ is equivalent to the relation $\epsilon^{0}=\bar{\epsilon}$. Then Theorem 4.4.8 and Propositions 3.1.6 and 3.1.7 yield the following statement.

Proposition 2.4. $1^{\circ}$. If the columns of the matrix $\epsilon(\mathrm{x})$ are solenoidal, then convergence in (2.26) and (2.28) is strong.

$2^{\circ}$. If the columns of the matrix $\epsilon(\mathbf{x})^{-1}$ are potential (up to an additive constant), then convergence in (2.27) is strong. If, moreover, $\nu(\mathbf{x})=$ const, then convergence in $(2.25)$ is also strong.

\section{§3. Homogenization for a Periodic Maxwell system with $\mu=1$}

3.1. Statement of the problem. We apply the results of $\S 2$ to the homogenization problem for the periodic Maxwell system. (Herewith, it suffices to assume that $\nu=1$ in the definition $(2.2)$ for $\mathcal{L}(\epsilon, \nu)$.) We assume that the magnetic permeability $\mu$ is equal to 1 . In what follows, $\mathbf{u}$ and $\mathbf{v}$ stand for the strength of the electric and the magnetic field, respectively. The dielectric permittivity $\epsilon(\mathbf{x})$ is a $\Gamma$-periodic $(3 \times 3)$-matrix-valued function with real entries and satisfying (2.1). Next, $\mathbf{w}=\epsilon \mathbf{u}$ is the electric displacement vector (the magnetic displacement vector is equal to $\mathbf{v}$, because $\mu=1$ ). We denote $\mathfrak{G}=L_{2}\left(\mathbb{R}^{3} ; \mathbb{C}^{3}\right)$.

Written in terms of the displacement vectors, the Maxwell operator $\mathcal{M}=\mathcal{M}(\epsilon)$ acts in the space

$$
\widetilde{\mathfrak{G}}=J \oplus J, \quad J=J\left(\mathbb{R}^{3}\right),
$$

and is defined by the formula

$$
\mathcal{M}(\epsilon) \operatorname{col}(\mathbf{w}, \mathbf{v})=\operatorname{col}\left(i \operatorname{rot} \mathbf{v},-i \operatorname{rot}(\epsilon(\mathbf{x}))^{-1} \mathbf{w}\right)
$$

on the domain

$$
\operatorname{Dom} \mathcal{M}(\epsilon)=\left\{\operatorname{col}(\mathbf{w}, \mathbf{v}): \mathbf{w} \in J, \operatorname{rot} \epsilon^{-1} \mathbf{w} \in \mathfrak{G}, \mathbf{v} \in J^{1}\right\} .
$$

The conditions $\mathbf{w} \in J$ and $\mathbf{v} \in J^{1}$ automatically supplement relations (3.1) with the equations

$$
\operatorname{div} \mathbf{w}=\operatorname{div} \mathbf{v}=0 .
$$

The operator $\mathcal{M}(\epsilon)$ is closed in $\widetilde{\mathfrak{G}}$, but is not selfadjoint.

We introduce the operator $\mathcal{M}_{\varepsilon}(\epsilon), \varepsilon>0$, acting in $\widetilde{\mathfrak{G}}$ by the formula

$$
\mathcal{M}_{\varepsilon}(\epsilon)=\mathcal{M}\left(\epsilon^{\varepsilon}\right) .
$$

The domain $\operatorname{Dom} \mathcal{M}_{\varepsilon}$ is given by the relations

$$
\mathbf{v} \in J^{1}, \quad \mathbf{w} \in J, \quad \operatorname{rot}\left(\epsilon^{\varepsilon}\right)^{-1} \mathbf{w} \in \mathfrak{G}
$$

and, consequently, depends on $\varepsilon$. 
Our goal is to study the behavior of the resolvent $\left(\mathcal{M}_{\varepsilon}-i I\right)^{-1}$ as $\varepsilon \rightarrow 0$. Consider the equation

$$
\left(\mathcal{M}_{\varepsilon}-i I\right) \operatorname{col}\left(\mathbf{w}_{\varepsilon}, \mathbf{v}_{\varepsilon}\right)=\operatorname{col}(\mathbf{q}, \mathbf{r}), \quad \mathbf{q}, \mathbf{r} \in J^{-1} .
$$

In detailed writing, (3.3) has the form

$$
\begin{aligned}
i \operatorname{rot} \mathbf{v}_{\varepsilon}-i \mathbf{w}_{\varepsilon} & =\mathbf{q}, \\
-i \operatorname{rot}\left(\epsilon^{\varepsilon}\right)^{-1} \mathbf{w}_{\varepsilon}-i \mathbf{v}_{\varepsilon} & =\mathbf{r}, \\
\operatorname{div} \mathbf{v}_{\varepsilon} & =0, \\
\operatorname{div} \mathbf{w}_{\varepsilon} & =0 .
\end{aligned}
$$

It is convenient (cf. $\S 6.3$ ) to represent the solutions $\mathbf{w}_{\varepsilon}, \mathbf{v}_{\varepsilon}$ as the sums

$$
\begin{aligned}
& \mathbf{w}_{\varepsilon}=\mathbf{w}_{\varepsilon}^{(q)}+\mathbf{w}_{\varepsilon}^{(r)}, \\
& \mathbf{v}_{\varepsilon}=\mathbf{v}_{\varepsilon}^{(q)}+\mathbf{v}_{\varepsilon}^{(r)},
\end{aligned}
$$

where $\operatorname{col}\left(\mathbf{w}_{\varepsilon}^{(q)}, \mathbf{v}_{\varepsilon}^{(q)}\right)$ is the solution of system (3.4) with $\mathbf{r}=0$, and $\operatorname{col}\left(\mathbf{w}_{\varepsilon}^{(r)}, \mathbf{v}_{\varepsilon}^{(r)}\right)$ is the solution of (3.4) with $\mathbf{q}=0$.

3.2. The case where $\mathbf{q}=0$. For $\operatorname{col}\left(\mathbf{w}_{\varepsilon}^{(r)}, \mathbf{v}_{\varepsilon}^{(r)}\right)$, system (3.4) takes the form

$$
\begin{aligned}
\mathbf{w}_{\varepsilon}^{(r)} & =\operatorname{rot} \mathbf{v}_{\varepsilon}^{(r)}, \\
\operatorname{rot}\left(\epsilon^{\varepsilon}\right)^{-1} \operatorname{rot}_{\mathbf{v}_{\varepsilon}}^{(r)}+\mathbf{v}_{\varepsilon}^{(r)} & =i \mathbf{r}, \\
\operatorname{div} \mathbf{v}_{\varepsilon}^{(r)} & =0 .
\end{aligned}
$$

Therefore,

$$
\mathbf{v}_{\varepsilon}^{(r)}=i\left(\mathcal{L}_{J, \varepsilon}(\epsilon)+I\right)^{-1} \mathbf{r},
$$

where $\mathcal{L}_{J, \varepsilon}(\epsilon)$ is the operator defined in Subsection 2.3. The displacement vector $\mathbf{w}_{\varepsilon}^{(r)}$ can be found from the relation

$$
\mathbf{w}_{\varepsilon}^{(r)}=\operatorname{rot} \mathbf{v}_{\varepsilon}^{(r)}=i \operatorname{rot}\left(\mathcal{L}_{J, \varepsilon}(\epsilon)+I\right)^{-1} \mathbf{r},
$$

while the strength $\mathbf{u}_{\varepsilon}^{(r)}$ is expressed in terms of the flow for $\mathbf{v}_{\varepsilon}^{(r)}$ :

$$
\mathbf{u}_{\varepsilon}^{(r)}=\left(\epsilon^{\varepsilon}\right)^{-1} \mathbf{w}_{\varepsilon}^{(r)}=\left(\epsilon^{\varepsilon}\right)^{-1} \operatorname{rot} \mathbf{v}_{\varepsilon}^{(r)} .
$$

Let $\mathbf{v}_{0}^{(r)}=i\left(\mathcal{L}_{J}^{0}+I\right)^{-1} \mathbf{r}$, i.e., $\mathbf{v}_{0}^{(r)}$ is the solution of the problem

$$
\operatorname{rot}\left(\epsilon^{0}\right)^{-1} \operatorname{rot} \mathbf{v}_{0}^{(r)}+\mathbf{v}_{0}^{(r)}=i \mathbf{r}, \quad \operatorname{div} \mathbf{v}_{0}^{(r)}=0 .
$$

We put

$$
\mathbf{w}_{0}^{(r)}=\operatorname{rot} \mathbf{v}_{0}^{(r)}, \quad \mathbf{u}_{0}^{(r)}=\left(\epsilon^{0}\right)^{-1} \operatorname{rot} \mathbf{v}_{0}^{(r)} .
$$

On the basis of (3.8)-(3.10) and Theorems 2.1-2.3, we obtain the following result.

Theorem 3.1. The following is true for the solutions of system (3.7).

$1^{\circ}$. If $\mathbf{r} \in J$, then $\mathbf{v}_{\varepsilon}^{(r)}$ converges in $\mathfrak{G}$ to $\mathbf{v}_{0}^{(r)}$, and

$$
\left\|\mathbf{v}_{\varepsilon}^{(r)}-\mathbf{v}_{0}^{(r)}\right\|_{\mathfrak{G}} \leq C \varepsilon\|\mathbf{r}\|_{\mathfrak{G}}, \quad 0<\varepsilon \leq 1
$$

$2^{\circ}$. If $\mathbf{r} \in J^{-s}, 0 \leq s<1$, then $\mathbf{v}_{\varepsilon}^{(r)}$ converges in $\mathfrak{G}^{s}$ to $\mathbf{v}_{0}^{(r)}$, and

$$
\left\|\mathbf{v}_{\varepsilon}^{(r)}-\mathbf{v}_{0}^{(r)}\right\|_{\mathfrak{G}^{s}} \leq C \varepsilon^{1-s}\|\mathbf{r}\|_{\mathfrak{G}^{-s}}, \quad 0<\varepsilon \leq 1
$$

$3^{\circ}$. If $\mathbf{r} \in J^{-1}$, then, as $\varepsilon \rightarrow 0, \mathbf{v}_{\varepsilon}^{(r)}$ converges to $\mathbf{v}_{0}^{(r)}$ weakly in $\mathfrak{G}^{1}$.

$4^{\circ}$. If $\mathbf{r} \in J^{-1}$, then, as $\varepsilon \rightarrow 0, \mathbf{w}_{\varepsilon}^{(r)}$ converges to $\mathbf{w}_{0}^{(r)}$ weakly in $\mathfrak{G}$, and $\mathbf{u}_{\varepsilon}^{(r)}$ converges to $\mathbf{u}_{0}^{(r)}$ weakly in $\mathfrak{G}$. 
We note also that the second equation in (3.7) implies

$$
\operatorname{rot} \mathbf{u}_{\varepsilon}^{(r)}=i \mathbf{r}-\mathbf{v}_{\varepsilon}^{(r)} .
$$

Similarly, $\operatorname{rot} \mathbf{u}_{0}^{(r)}=i \mathbf{r}-\mathbf{v}_{0}^{(r)}$. Consequently,

$$
\left\|\operatorname{rot} \mathbf{u}_{\varepsilon}^{(r)}-\operatorname{rot} \mathbf{u}_{0}^{(r)}\right\|_{\mathfrak{G}} \leq C \varepsilon\|\mathbf{r}\|_{\mathfrak{G}}, \quad \mathbf{r} \in J, \quad 0<\varepsilon \leq 1 .
$$

The following result is a consequence of Proposition 2.4.

Proposition 3.2. If the columns of the matrix $\epsilon(\mathbf{x})^{-1}$ are potential, then $\mathbf{v}_{\varepsilon}^{(r)}$ converges to $\mathbf{v}_{0}^{(r)}$ strongly in $\mathfrak{G}^{1}$. If the columns of the matrix $\epsilon(\mathbf{x})$ are solenoidal, then $\mathbf{u}_{\varepsilon}^{(r)}$ converges to $\mathbf{u}_{0}^{(r)}$ strongly in $\mathfrak{G}$.

3.3. The case where $\mathbf{r}=0$. For $\operatorname{col}\left(\mathbf{w}_{\varepsilon}^{(q)}, \mathbf{v}_{\varepsilon}^{(q)}\right)$, system (3.4) has the form

$$
\begin{aligned}
i \operatorname{rot} \mathbf{v}_{\varepsilon}^{(q)}-i \mathbf{w}_{\varepsilon}^{(q)} & =\mathbf{q}, \\
\operatorname{rot}\left(\epsilon^{\varepsilon}\right)^{-1} \mathbf{w}_{\varepsilon}^{(q)}+\mathbf{v}_{\varepsilon}^{(q)} & =0, \\
\operatorname{div} \mathbf{v}_{\varepsilon}^{(q)} & =0, \\
\operatorname{div} \mathbf{w}_{\varepsilon}^{(q)} & =0 .
\end{aligned}
$$

In this subsection we restrict ourselves to the case where $\mathbf{q} \in J$.

Lemma 3.3. Let $\mathbf{q} \in J$. Then the solutions of system (3.11) satisfy the inequalities

$$
\begin{aligned}
\left\|\mathbf{v}_{\varepsilon}^{(q)}\right\|_{\mathfrak{G}^{1}} & \leq C\|\mathbf{q}\|_{\mathfrak{G}}, \\
\left\|\mathbf{w}_{\varepsilon}^{(q)}\right\|_{\mathfrak{G}} & \leq C\|\mathbf{q}\|_{\mathfrak{G} .} .
\end{aligned}
$$

Therefore, for $\mathbf{u}_{\varepsilon}^{(q)}=\left(\epsilon^{\varepsilon}\right)^{-1} \mathbf{w}_{\varepsilon}^{(q)}$ we have

$$
\left\|\mathbf{u}_{\varepsilon}^{(q)}\right\|_{\mathfrak{G}} \leq C\|\mathbf{q}\|_{\mathfrak{G}}
$$

Proof. We multiply the second equation in (3.11) by $\mathbf{v}_{\varepsilon}^{(q)}$ and integrate:

$$
\left(\left(\epsilon^{\varepsilon}\right)^{-1} \mathbf{w}_{\varepsilon}^{(q)}, \operatorname{rot} \mathbf{v}_{\varepsilon}^{(q)}\right)_{\mathfrak{G}}+\left\|\mathbf{v}_{\varepsilon}^{(q)}\right\|_{\mathfrak{G}}^{2}=0 .
$$

The first equation yields

$$
\mathbf{w}_{\varepsilon}^{(q)}=\operatorname{rot} \mathbf{v}_{\varepsilon}^{(q)}+i \mathbf{q}
$$

substituting, we obtain

$$
\left(\left(\epsilon^{\varepsilon}\right)^{-1} \operatorname{rot} \mathbf{v}_{\varepsilon}^{(q)}, \operatorname{rot} \mathbf{v}_{\varepsilon}^{(q)}\right)_{\mathfrak{G}}+\left\|\mathbf{v}_{\varepsilon}^{(q)}\right\|_{\mathfrak{G}}^{2}=-i\left(\left(\epsilon^{\varepsilon}\right)^{-1} \mathbf{q}, \operatorname{rot} \mathbf{v}_{\varepsilon}^{(q)}\right)_{\mathfrak{G}} .
$$

Combining this with the relation $\operatorname{div} \mathbf{v}_{\varepsilon}^{(q)}=0$, we arrive at (3.12). Estimate (3.13) follows from (3.12) and (3.14).

By Lemma 3.3, in the proof of the weak $\left(\mathfrak{G}^{1}\right)$-convergence of $\mathbf{v}_{\varepsilon}^{(q)}$ and the weak (G) convergence of $\mathbf{w}_{\varepsilon}^{(q)}$ and $\mathbf{u}_{\varepsilon}^{(q)}$ we may assume that

$$
\mathbf{q}=\operatorname{rot} \mathbf{F}, \quad \mathbf{F} \in J^{1} .
$$

We put

$$
\boldsymbol{\Phi}_{\varepsilon}^{(q)}=i\left(\mathcal{L}_{J, \varepsilon}(\epsilon)+I\right)^{-1} \mathbf{F} .
$$

It is easy to check that the functions

$$
\begin{aligned}
& \mathbf{w}_{\varepsilon}^{(q)}=\operatorname{rot} \boldsymbol{\Phi}_{\varepsilon}^{(q)}, \\
& \mathbf{v}_{\varepsilon}^{(q)}=\boldsymbol{\Phi}_{\varepsilon}^{(q)}-i \mathbf{F}
\end{aligned}
$$


satisfy system (3.11) with q defined by (3.15). Then, by (3.17),

$$
\mathbf{u}_{\varepsilon}^{(q)}=\left(\epsilon^{\varepsilon}\right)^{-1} \operatorname{rot} \boldsymbol{\Phi}_{\varepsilon}^{(q)},
$$

i.e., $\mathbf{u}_{\varepsilon}^{(q)}$ is expressed via the flow for $\boldsymbol{\Phi}_{\varepsilon}^{(q)}$. Furthermore, the second relation in (3.11) means that

$$
\operatorname{rot} \mathbf{u}_{\varepsilon}^{(q)}=-\mathbf{v}_{\varepsilon}^{(q)}
$$

Let

$$
\begin{gathered}
\boldsymbol{\Phi}_{0}^{(q)}=i\left(\mathcal{L}_{J}^{0}+I\right)^{-1} \mathbf{F}, \\
\mathbf{v}_{0}^{(q)}=\boldsymbol{\Phi}_{0}^{(q)}-i \mathbf{F}, \\
\mathbf{w}_{0}^{(q)}=\operatorname{rot} \boldsymbol{\Phi}_{0}^{(q)}, \quad \mathbf{u}_{0}^{(q)}=\left(\epsilon^{0}\right)^{-1} \operatorname{rot} \boldsymbol{\Phi}_{0}^{(q)} .
\end{gathered}
$$

Theorem 2.3 and (3.16)-(3.23) imply the following theorem.

Theorem 3.4. Let $\mathbf{q} \in J$. Then, as $\varepsilon \rightarrow 0$,

1) the fields $\mathbf{v}_{\varepsilon}^{(q)}$ converge to $\mathbf{v}_{0}^{(q)}$ weakly in $\mathfrak{G}^{1}$;

2) the fields $\mathbf{w}_{\varepsilon}^{(q)}$ converge to $\mathbf{w}_{0}^{(q)}$ weakly in $\mathfrak{G}$;

3) the fields $\mathbf{u}_{\varepsilon}^{(q)}$ converge to $\mathbf{u}_{0}^{(q)}$ weakly in $\mathfrak{G}$, and $\operatorname{rot} \mathbf{u}_{\varepsilon}^{(q)}$ converges to $\operatorname{rot} \mathbf{u}_{0}^{(q)}$ weakly in $\mathfrak{G}^{1}$.

Moreover, we have the following consequence of Proposition 2.4.

Proposition 3.5. If the columns of the matrix $\epsilon(\mathbf{x})^{-1}$ are potential, then the fields $\mathbf{v}_{\varepsilon}^{(q)}$ converge to $\mathbf{v}_{0}^{(q)}$ strongly in $\mathfrak{G}^{1}$. If the columns of the matrix $\epsilon(\mathbf{x})$ are solenoidal, then the fields $\mathbf{u}_{\varepsilon}^{(q)}$ converge to $\mathbf{u}_{0}^{(q)}$ strongly in $\mathfrak{G}$.

Now, we apply Theorems 2.1 and 2.2 to functions (3.16), and take (3.18) into account. Then, for $\mathbf{F} \in J^{1}$ and $\mathbf{q}=\operatorname{rot} \mathbf{F}$, we have

$$
\begin{aligned}
\left\|\mathbf{v}_{\varepsilon}^{(q)}-\mathbf{v}_{0}^{(q)}\right\|_{\mathfrak{G}} & \leq C \varepsilon\|\mathbf{F}\|_{\mathfrak{G}}, & & 0<\varepsilon \leq 1, \\
\left\|\mathbf{v}_{\varepsilon}^{(q)}-\mathbf{v}_{0}^{(q)}\right\|_{\mathfrak{G}^{s}} & \leq C \varepsilon^{1-s}\|\mathbf{F}\|_{\mathfrak{G}^{-s}}, & & 0 \leq s<1, \quad 0<\varepsilon \leq 1 .
\end{aligned}
$$

These relations and estimate (3.12) yield the $\left(\mathfrak{G}^{s}\right)$-convergence of $\mathbf{v}_{\varepsilon}^{(q)}$ to $\mathbf{v}_{0}^{(q)}$ for any $\mathbf{q} \in J$, but already without a "qualified" estimate. The latter feature is related to the fact that condition (3.15) does not imply any estimate of the norm $\|\mathbf{F}\|_{\mathfrak{G}}$ in terms of $\|\mathbf{q}\|_{\mathfrak{G}}$. Thus, we arrive at the following statement.

Theorem 3.6. If $\mathbf{q} \in J$, then, as $\varepsilon \rightarrow 0$, the fields $\mathbf{v}_{\varepsilon}^{(q)}$ converge to $\mathbf{v}_{0}^{(q)}$ strongly in $\mathfrak{G}^{s}$, $0 \leq s<1$.

3.4. As we have seen, the quality of convergence for separate summands can be better than for the sums (3.5) and (3.6). Still, we formulate the summarizing result. Theorems $3.1,3.4$, and 3.6 lead to the following conclusion.

Theorem 3.7. Let $\mathcal{M}^{0}=\mathcal{M}\left(\epsilon^{0}\right)$ be the effective Maxwell operator defined by (3.1) and (3.2) with $\epsilon(\mathbf{x})$ replaced by $\epsilon^{0}$. Let

$$
\operatorname{col}\left(\mathbf{w}_{\varepsilon}, \mathbf{v}_{\varepsilon}\right)=\left(\mathcal{M}_{\varepsilon}-i I\right)^{-1} \operatorname{col}(\mathbf{q}, \mathbf{r}), \quad \operatorname{col}\left(\mathbf{w}_{0}, \mathbf{v}_{0}\right)=\left(\mathcal{M}_{0}-i I\right)^{-1} \operatorname{col}(\mathbf{q}, \mathbf{r}),
$$

where $\mathbf{q} \in J, \mathbf{r} \in J$, and let

$$
\mathbf{u}_{\varepsilon}=\left(\epsilon^{\varepsilon}\right)^{-1} \mathbf{w}_{\varepsilon}, \quad \mathbf{u}_{0}=\left(\epsilon^{0}\right)^{-1} \mathbf{w}_{0} .
$$

Then, as $\varepsilon \rightarrow 0$,

1) the fields $\mathbf{v}_{\varepsilon}$ converge to $\mathbf{v}_{0}$ strongly in $\mathfrak{G}^{s}, 0 \leq s<1$, and weakly in $\mathfrak{G}^{1}$; 
2) the fields $\mathbf{w}_{\varepsilon}$ converge to $\mathbf{w}_{0}$ weakly in $\mathfrak{G}$;

3) the fields $\mathbf{u}_{\varepsilon}$ converge to $\mathbf{u}_{0}$ weakly in $\mathfrak{G}$, and $\operatorname{rot} \mathbf{u}_{\varepsilon}$ converges to rot $\mathbf{u}_{0}$ strongly in $\mathfrak{G}$.

Combining Propositions 3.2 and 3.5, we obtain the following.

Proposition 3.8. If the columns of the matrix $\epsilon(\mathbf{x})^{-1}$ are potential, then the fields $\mathbf{v}_{\varepsilon}$ converge to $\mathbf{v}_{0}$ strongly in $\mathfrak{G}^{1}$. If the columns of the matrix $\epsilon(\mathbf{x})$ are solenoidal, then the fields $\mathbf{u}_{\varepsilon}$ converge to $\mathbf{u}_{0}$ strongly in $\mathfrak{G}$.

\section{Comments on Chapter 7}

1. The results of Theorems $2.1,2.2$ about homogenization applied to the operator $\mathcal{L}_{J, \varepsilon}(\epsilon)$ are new. Relation (2.27) in Theorem 2.3 is close to some results of [BeLP]. We dwell on this in more detail.

In $\overline{B e L P}$, an equation of type (2.23) (but in a bounded domain and with appropriate boundary conditions) was called a "Maxwell type" equation. The condition $\operatorname{div} \mathbf{v}_{\varepsilon}=0$ was not assumed, but if $\operatorname{div} \mathbf{F}=0$, it is fulfilled automatically. Extension of the system to an elliptic one was not employed. Up to these minor differences, our statement about the limit (2.27) repeats the result of [BeLP]. In [BeLP], convergence of flows was not discussed. Therefore, our relation (2.28) is of certain interest: it is applied in $\S 3$ to the homogenization problem for the Maxwell system itself.

2. Application of the results to the stationary Maxwell system was not discussed in BeLP. However, along with (2.23), the more general system

$$
\operatorname{rot}\left(\epsilon^{\varepsilon}\right)^{-1} \operatorname{rot} \mathbf{v}_{\varepsilon}+a^{\varepsilon} \mathbf{v}_{\varepsilon}=\mathbf{F}
$$

was considered in that paper. It was proved that the solutions $\mathbf{v}_{\varepsilon}$ converge weakly in the space with the metric form $\|\operatorname{rot} \mathbf{v}\|_{L_{2}}^{2}+\|\mathbf{v}\|_{L_{2}}^{2}$. In the limit equation, $a$ is also homogenized by the acoustic rule. The condition $\operatorname{div} \mathbf{F}=0$ automatically implies that $\operatorname{div} a^{\varepsilon} \mathbf{v}_{\varepsilon}=0$. Application to the stationary Maxwell system was not discussed.

Equation (+) is not covered by our scheme. Theorem 4.4.1 is not applicable; moreover, the homogenization rule for the lower term given by this theorem does not agree with the acoustic rule.

3. Homogenization for the stationary and nonstationary Maxwell systems was studied in [ZhKO]. In both cases, the solenoidal conditions were not included in the system. The weak $L_{2}$-convergence of solutions to the solution of the limit equation was established in [ZhKO]; both electric permittivity and magnetic permeability were homogenized by the acoustic rule. Some earlier and weaker results about the nonstationary Maxwell system can be found in $\mathrm{Sa}, \mathrm{BeLP}$.

4. We recall that, in our approach to the homogenization problem for the Maxwell system, two simplifying assumptions were made: 1) $\mu(\mathbf{x})=0$ and 2) the solenoidal conditions are included in the system. Besides, the splitting representation of the fields into the sum of two terms turned out to be rather useful.

\section{CONCLUding REMARKS}

1. As compared to [BSu2], the abstract basis enriched in the present paper. New Theorems 1.5.8 and 1.5.9 have appeared, along with the fact that all constants in estimates are controlled carefully. As applied to homogenization, this has led to felicitous Theorems 4.2.5 and 4.2.6. 
2. As has already been mentioned, in homogenization theory it is more traditional to consider, e.g., equations of the form $\mathcal{A}_{\varepsilon}(g, f)=\mathbf{F}$ in a fixed bounded domain $\mathcal{O}$ with appropriate boundary conditions. As compared to the case of the entire space $\mathbb{R}^{d}$, there are some differences. On the one hand, the compactness of the embedding of $H^{1}(\mathcal{O})$ in $L_{2}(\mathcal{O})$ facilitates the study. On the other hand, the effects near $\partial \mathcal{O}$ may hinder obtaining a good estimate for the difference $\mathbf{u}_{\varepsilon}-\mathbf{u}_{0}$ (see [ZhKO]).

3. Homogenization problems can be related not only to the lower edge of the spectrum, but also to the edges of internal gaps. Then, a shift to the high energy area arizes necessarily. Thus, a homogenization effects begin to interact with high-energy ones. For the case of the simplest model, this phenomenon was discussed in the recent paper [B3].

4. Throughout the text, the authors tried to distinguish the cases where weak convergence becomes strong. There is no need to explain that "the weak limit is much weaker than the strong one". In this connection, we mention that, e.g., in the problem considered in [B3], the weak limit always exists but is equal to zero. In this case, the $\varepsilon$-depending approximations of solutions turn out to be informative.

5. In homogenization theory, many specific methods are well developed. The idea to apply the methods of analytic perturbation theory (on the basis of the Floquet-Bloch decomposition) stands somewhat apart. However, this idea itself is not new at all. Some material of this kind can be found in BeLP, Chapter 4] and [ZhKO, Chapter 2]. Apparently, this way was not employed consistently and intensively enough. We think that what was missing is an exact definition of a threshold effect. Also, the class of operators admitting an appropriate factorization was not distinguished. Finally, the abstract operator theory basis of threshold effects was not analyzed. For these reasons, the present paper has almost no direct intersections with the papers by other authors that used the Floquet-Bloch decomposition and perturbation theory. Among such papers, we have already mentioned the remarkable paper [Zh1]. The paper [Se], where the acoustic operator with $g \in C^{\infty}$ and $d \geq 3$ was considered, also deserves special mention. With the help of perturbation theory, the full asymptotic expansion in powers of $\varepsilon$ was obtained in [Se] for the solution of the equation $\mathbf{D}^{*} g\left(\varepsilon^{-1} \mathbf{x}\right) \mathbf{D} u=F \in C_{0}^{\infty}\left(\mathbb{R}^{d}\right)$. In [CV], for the acoustic operator, formula (5.1.9) (in our notation) was proved, where the left-hand side was defined in terms of perturbation theory, and $g^{0}$ on the right-hand side was the effective matrix arising in the classical homogenization theory. In all these papers $n=1$, which significantly simplifies the arguments.

6. The applicability limits. The number of examples could be extended substantially. Besides periodic operators in $\mathbb{R}^{d}$, we can study periodic problems in domains of cylinder or layer type, etc., on the same abstract basis. In such problems, the momentum dimension is less than the coordinate dimension. This leads to new phenomena in the study of the effective characteristics and to other technical difficulties. An informative example of this kind was discussed in $[\mathrm{Su}]$.

We now indicate some cases where our method is not applicable.

1) Consider the matrix Schrödinger operator $H_{n}=-\Delta+p(\mathbf{x})$ in $L_{2}\left(\mathbb{R}^{d} ; \mathbb{C}^{n}\right)$, where $p$ is a periodic Hermitian $(n \times n)$-matrix-valued potential. In this case we have no analog of the factorization (6.1.6). Assume that $\lambda=0$ is the lower edge of the spectrum of $H_{n}$, and let $H_{n}(\mathbf{k})$ be the corresponding operator in $L_{2}\left(\Omega ; \mathbb{C}^{n}\right)$. Now, the point $\mathbf{k}=0$ does not play any distinguished role. It may happen that $\operatorname{Ker} H_{n}=\{0\}$. As a result, the possibility to study the threshold properties efficiently is lost. 
2) The periodic Pauli operator for $d=3$ is defined by the formula $\mathcal{D}_{3}=\left(D_{1}-A_{1}\right) \sigma_{1}+$ $\left(D_{2}-A_{2}\right) \sigma_{2}+\left(D_{3}-A_{3}\right) \sigma_{3}$. For this operator, there is no analog of the factorization (6.2.12). Moreover, we cannot describe the kernel $\operatorname{Ker} \mathcal{D}_{3}(0)$ in reasonable terms.

3) The magnetic periodic Schrödinger operator is defined by the formula $\mathcal{M}=(\mathbf{D}-$ $\mathbf{A}(\mathbf{x}))^{*}(\mathbf{D}-\mathbf{A}(\mathbf{x})), d \geq 2$, where $\operatorname{div} \mathbf{A}=0, \int_{\Omega} \mathbf{A} d \mathbf{x}=0$. Now the lower edge of the spectrum is a positive number. After shifting the lower edge to zero, factorization is lost. The question as to whether the factorization can be recovered is not easy. If the magnetic potential is not too large, the answer is in the positive; otherwise serious complications may occur even for $d=2$. The corresponding analysis was performed recently in [Sh].

\section{REFERENCES}

[BaPa] N. S. Bakhvalov and G. P. Panasenko, Homogenization: averaging processes in periodic media. Mathematical problems in the mechanics of composite materials, "Nauka", Moscow, 1984; English transl., Math. Appl. (Soviet Ser.), vol. 36, Kluwer Acad. Publ. Group, Dordrecht, 1989. MR 0797571 (86m:73049)

[BeLP] A. Bensoussan, J.-L. Lions, and G. Papanicolaou, Asymptotic analysis for periodic structures, Stud. Math. Appl., vol. 5, North-Holland Publishing Co., Amsterdam-New York, 1978. MR 0503330 (82h:35001)

[B1] M. Sh. Birman, Discrete spectrum of the periodic Schrödinger operator perturbed by a decaying potential, Algebra i Analiz 8 (1996), no. 1, 3-20; English transl., St. Petersburg Math. J. 8 (1997), no. 1, 1-14. MR 1392011 (97h:47047)

[B2] Discrete spectrum in the gaps of a perturbed periodic Schrödinger operator. II. Nonregular perturbations, Algebra i Analiz 9 (1997), no. 6, 62-89; English transl., St. Petersburg Math. J. 9 (1998), no. 6, 1073-1095. MR 1610239 (99h:47054)

[B3] On homogenization procedure for periodic operators near the edge of an internal gap, Algebra i Analiz 15 (2003), no. 4, 61-71; English transl., St. Petersburg Math. J. 15 (2004), no. 4, 507-513.

[BLaSu] M. Sh. Birman, A. Laptev, and T. A. Suslina, Discrete spectrum of a two-dimensional periodic elliptic second order operator perturbed by a decaying potential. I. Semibounded gap, Algebra i Analiz 12 (2000), no. 4, 36-78; English transl., St. Petersburg Math. J. 12 (2001), no. 4, 535-567. MR 1793617 (2003b:47078)

[BSu1] M. Sh. Birman and T. A. Suslina, Two-dimensional periodic Pauli operator. The effective masses at the lower edge of the spectrum, Mathematical Results in Quantum Mechanics (Prague, 1998), Oper. Theory Adv. Appl., vol. 108, Birkhäuser, Basel, 1999, pp. 13-31. MR 1708785 (2000g:81049)

[BSu2] Threshold effects near the lower edge of the spectrum for periodic differential operators of mathematical physics, Systems, Approximation, Singular Integral Operators, and Related Topics (Bordeaux, 2000), Oper. Theory Adv. Appl., vol. 129, Birkhäuser, Basel, 2001, pp. 71107. MR 1882692 (2003f:35220)

[CV] C. Conca and M. Vanninathan, Homogenization of periodic structures via Bloch decomposition, SIAM J. Appl. Math. 57 (1997), no. 6, 1639-1659. MR 1484944 (98j:35017)

[Ka] T. Kato, Perturbation theory for linear operators, 2nd ed., Grundlehren Math. Wiss., vol. 132, Springer-Verlag, Berlin-New York, 1976. MR 0407617 (53:11389)

[KiSi] W. Kirsh and B. Simon, Comparison theorems for the gap of Schrödinger operators, J. Funct. Anal. 75 (1987), no. 2, 396-410. MR 0916759 (89b:35127)

[Ku] P. Kuchment, The mathematics of photonic crystals, Mathematical Modeling in Optical Science, Frontiers Appl. Math., vol. 22, SIAM, Philadelphia, PA, 2001, pp. 207-272. MR 1831334 (2002k:78002)

[Zh1] V. V. Zhikov, Spectral approach to asymptotic diffusion problems, Differentsial'nye Uravneniya 25 (1989), no. 1, 44-50; English transl., Differential Equations 25 (1989), no. 1, 33-39. MR 0986395 (90a:35107)

[Zh2] , Private communication, 2002. (Russian)

[ZhKO] V. V. Zhikov, S. M. Kozlov, and O. A. Oleŭnik, Homogenization of differential operators, "Nauka", Moscow, 1993; English transl., Springer-Verlag, Berlin, 1994. MR 1329546 (96h:35003b)

[Sa] E. Sanchez-Palencia, Nonhomogeneous media and vibration theory, Lecture Notes in Phys., vol. 127, Springer-Verlag, Berlin-New York, 1980. MR 0578345 (82j:35010) 
[Se] E. V. Sevost'yanova, Asymptotic expansion of the solution of a second-order elliptic equation with periodic rapidly oscillating coefficients, Mat. Sb. (N.S.) 115 (1981), no. 2, 204-222; English transl., Math. USSR-Sb. 43 (1982), no. 2, 181-198. MR 0622145 (83d:35038)

[Su $\quad$ T. A. Suslina, On homogenization of a periodic elliptic operator in a strip, Algebra i Analiz 16 (2004), no. 1, 269-292; English transl. in St. Petersburg Math. J. 16 (2005), no. 1 (to appear).

[Sh] R. G. Shterenberg, Example of a periodic magnetic Schrödinger operator with degenerate lower spectral edge, Algebra i Analiz (to appear). (Russian)

Department of Physics, St. Petersburg State University, Ul’yanovskaya 1, Petrodvorets, St. Petersburg 198504, Russia

Department of Physics, St. Petersburg State University, Ul'yanovskaya 1, Petrodvorets, St. Petersburg 198504, Russia

E-mail address: tanya@petrov.stoic.spb.su

Received 25/JUN/2003

Translated by T. A. SUSLINA 